\title{
Coping Mechanisms Among Youth and Young Adults with Autism Spectrum Disorder in Competitive Employment
}

by

\author{
Abirami Vijayakumar
}

A thesis submitted in conformity with the requirements for the degree of Master of Science

Rehabilitation Sciences Institute

University of Toronto

(C) Copyright by Abirami Vijayakumar 2021 


\title{
Coping Mechanisms Among Youth and Young Adults with Autism Spectrum Disorder in Competitive Employment
}

\author{
Abirami Vijayakumar \\ Master of Science \\ Rehabilitation Sciences Institute \\ University of Toronto
}

2021

\section{Abstract}

Successful integration into employment is an ongoing issue for individuals with autism spectrum disorder (ASD). A primary barrier to successful employment includes being unable to cope with the social demands of work because people with ASD are more likely to experience higher levels of stress and anxiety. The purpose of this study was to explore how youth and young adults with ASD cope with stress and anxiety in competitive employment. A phenomenological study was conducted involving in-depth interviews with twelve youth and young adults with ASD.

Findings revealed three main themes: (1) salient workplace factors, (2) stress and/or anxiety inducers, and (3) coping mechanisms. The findings shed new light on the impact of stress, anxiety and coping on youth and young adults with ASD and have potential to educate employers about the issues that such employees encounter. 


\section{Acknowledgments}

I would like to begin by thanking my supervisor, Dr. Sally Lindsay for giving me an opportunity to pursue graduate studies at the University of Toronto and for providing ongoing insight and guidance throughout my graduate degree journey. I am so thankful for the countless edits and feedback for thesis chapters and for helping me stick to my timeline. I have learned so much in these last two years and for that, I am so grateful. I would also like to thank my committee members Dr. Melanie Penner and Dr. Shannon Scratch for their unwavering support and guidance. They always answered my numerous questions and provided honest feedback throughout each phase of my thesis. Thank you for being a part of my committee!

This research was supported in part by the Canadian Institutes of Health Research, Social Sciences and Humanities Research, and the Kimmel Family funds from Holland Bloorview Kids Rehabilitation Hospital. I am grateful for their support.

I am incredibly thankful to all of the wonderful staff and students in the TRAIL lab for their friendship, encouragement and overall moral support throughout the years. Specifically, Hiba thank you for always being so kind and offering to help in any way possible!

To my incredible participants, I am so thankful for their insight for this wonderful project. Without their stories and experiences, this study would not be possible.

To my wonderful support system, I am beyond grateful and fortunate to have your love and support throughout this journey. Thansha, you are always supporting me in whatever decision I make. You are more than just family to me, you are my mentor and friend. Thank you for being a light in my life. Amreetha, you are one of a kind and I thank you for always lifting me up, especially when times are difficult. You always believe in me and I am so grateful to have a positive friend like you in my life. To Alana, my ride or die, best friend and sister, thank you for being the greatest friend a person can ask for. From the late night chats to going on socially distanced walks together, thank you so much for always encouraging me to be my best self...oh and for always making me laugh.

To my wonderful and amazing family, I am so blessed to have your support and guidance throughout these two years. Amma and Appa, thank you for everything that you have done for me. You are amazing parents, and I am so grateful to have a family that encourages me and lifts 
me up every single day. To Arun, I cannot thank you enough for always being there by my side. You are a wonderful brother who always encourages me to try my best and reach for the stars. You are always in my corner, believing in me, and I am forever grateful. To Aravinth, the gem of this family, the person who made me interested in this field of research, thank you for being my brother and for teaching me so many life lessons. I am truly so blessed to have so many loving people in my life.

Finally, I would like to thank God for continuously supporting me in my journey and for shaping me into the person I am today. 


\section{Table of Contents}

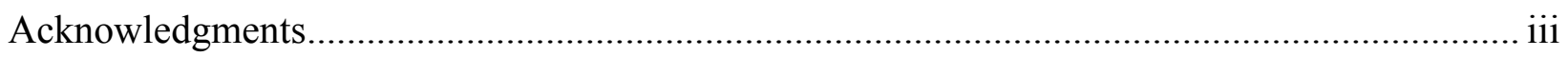

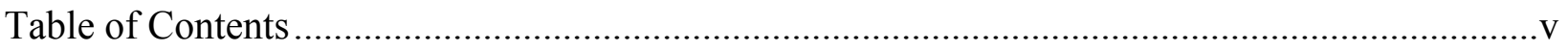

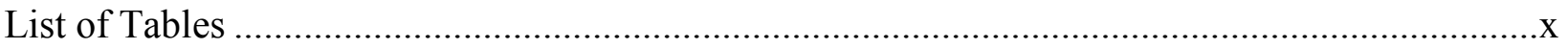

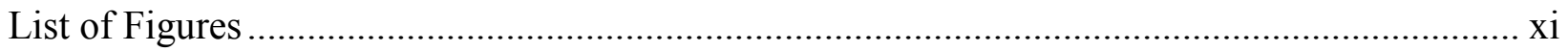

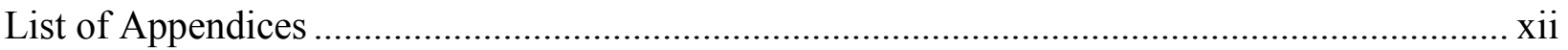

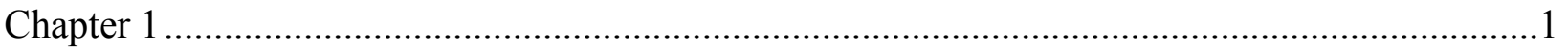

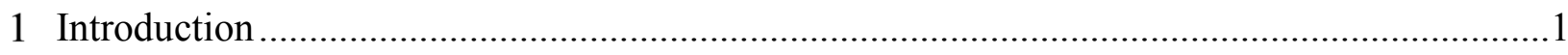

1.1 Brief Background:

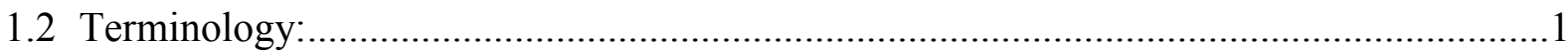

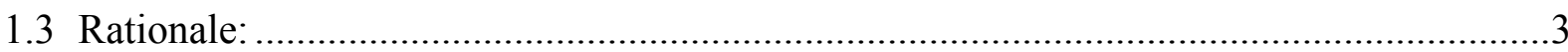

1.4 Research Question and Objectives: ............................................................................

1.5 Importance of this study and gaps addressed:_.............................................................6

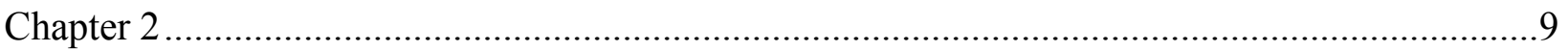

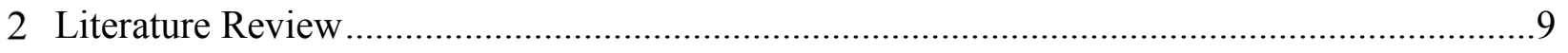

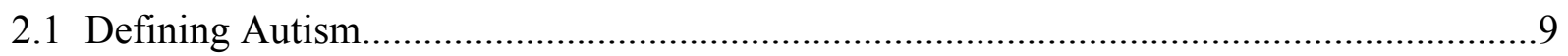

2.2 Employment for Youth and Young Adults with ASD ...................................................10

2.2.1 Employment Rates for People with ASD ………………………….................10

2.2.2 Employment Skills................................................................................11

2.2.3 Finding Employment, Disability Disclosure, and Accommodations in the Workplace ..................................................................................................12

2.2.4 Barriers to Employment ................................................................................14

2.2.5 Benefits of Hiring Inclusively ........................................................................

2.2.6 Positive Experiences with Employment Among People with ASD ......................18

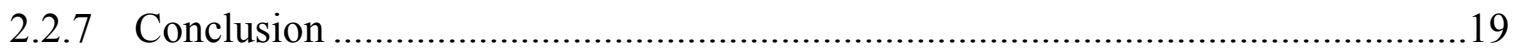

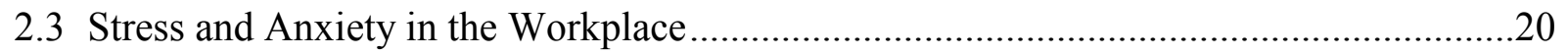




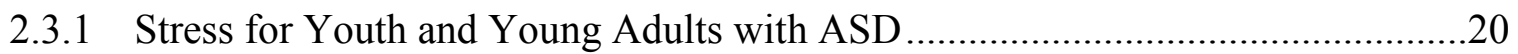

2.3.2 Anxiety for Youth and Young Adults with ASD.................................................21

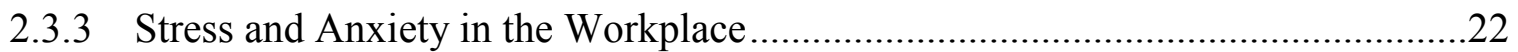

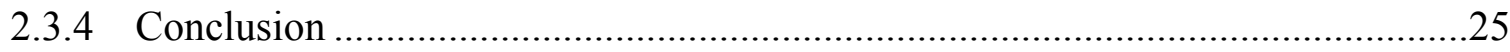

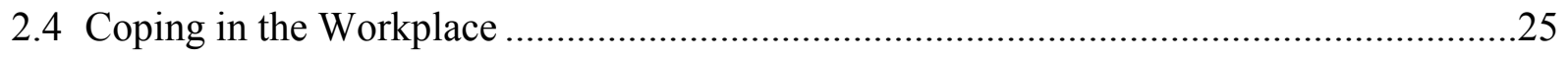

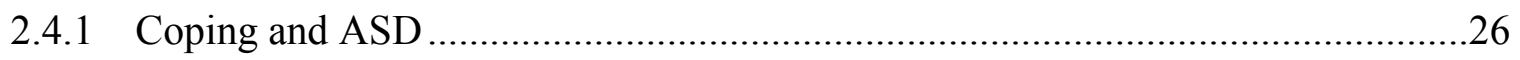

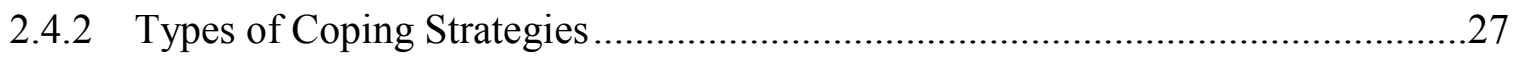

2.4.3 Coping in Employment ……………………..............................................28

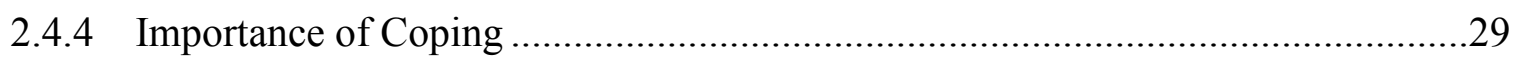

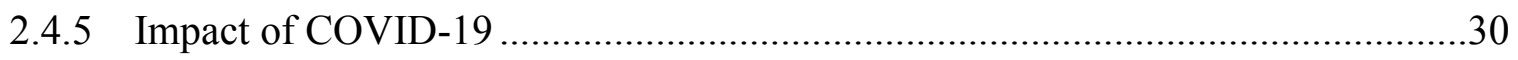

2.4.6 Potential Implications and Conclusion …………...............................................30

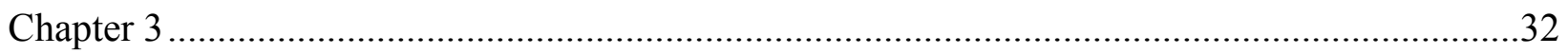

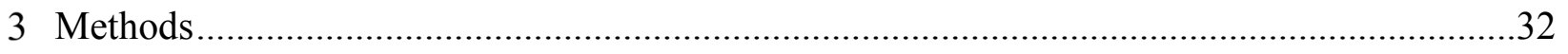

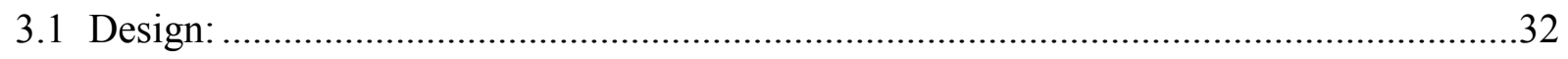

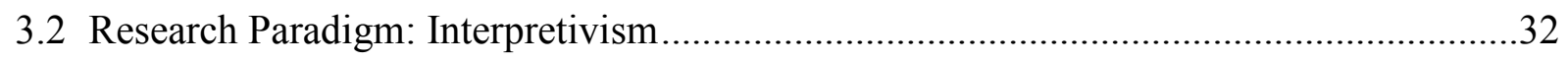

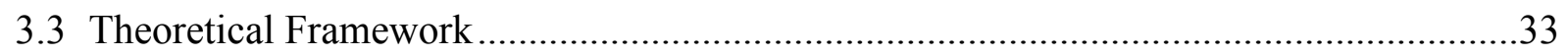

3.4 Research Methodology: Interpretative Phenomenological Analysis ...................................35

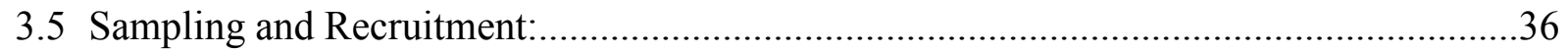

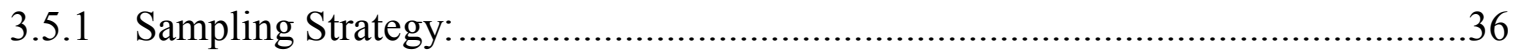

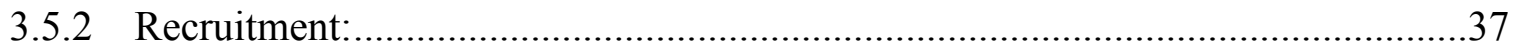

3.5.3 Inclusion Criteria for Study Sample: ...............................................................

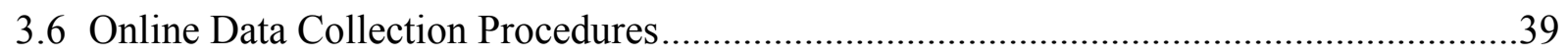

3.6.1 Utilizing Zoom to Conduct Interviews: .................................................................39

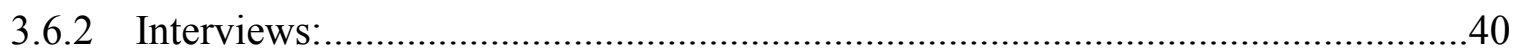

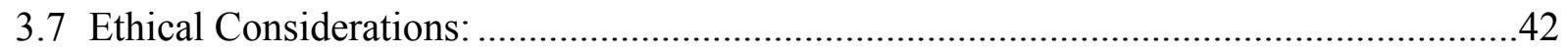

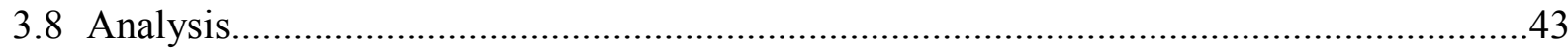


3.8.1 Approach to data analysis:

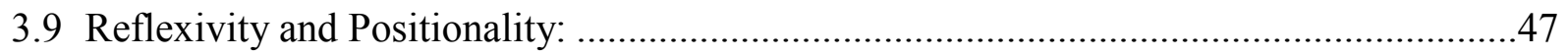

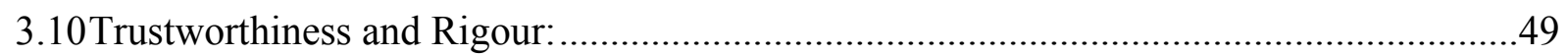

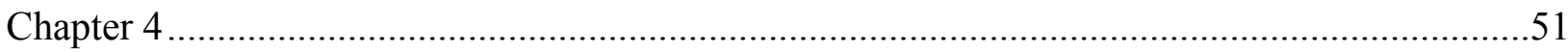

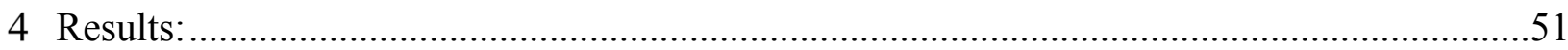

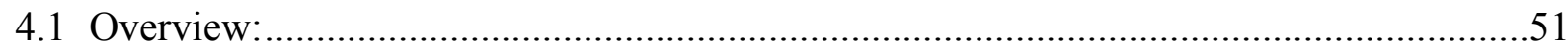

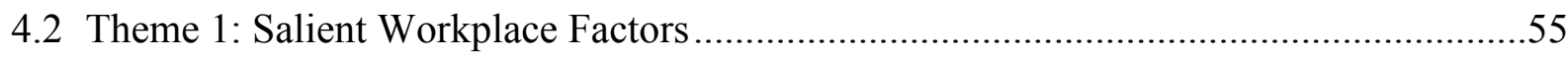

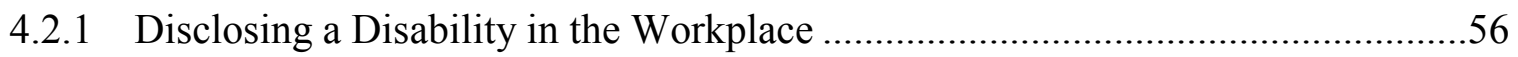

4.2.2 My Experience with Asking for Workplace Adjustments ................................59

4.2.3 My Workplace Environment..................................................................62

4.2.4 Challenges with my Coworkers or Supervisor ..........................................64

4.2.5 My Interests and Preferences Align with My Work ......................................66

4.2.6 Ways I Prevent Future Stress Occurrences ..................................................67

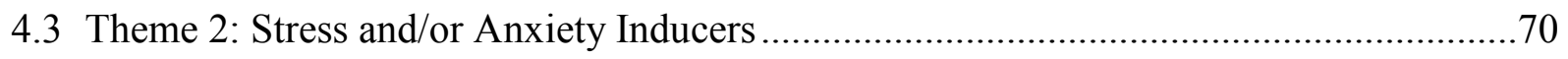

4.3.1 ASD-related Challenges: Impact of ASD Diagnosis on Employment

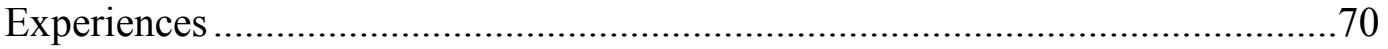

4.3.2 ASD-related Challenges: Challenges When I Communicate or Interact at

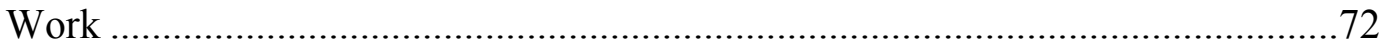

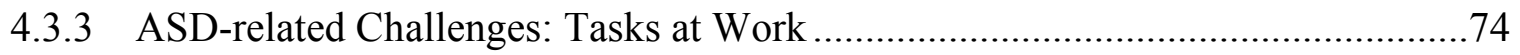

4.3.4 Past Trauma/Experience Impacts My Ability to Work.......................................76

4.3.5 My Stressful Experience with Disability Disclosure ......................................78

4.3.6 Impact of the First Wave of COVID-19 ....................................................... 79

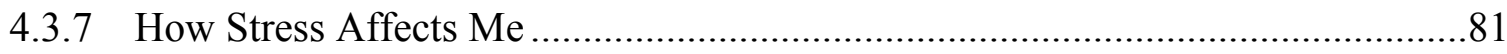

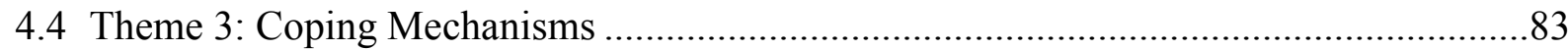

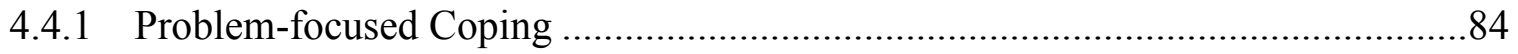

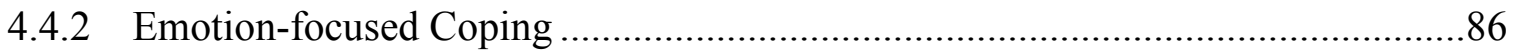

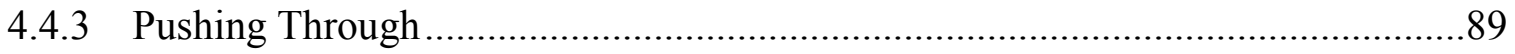




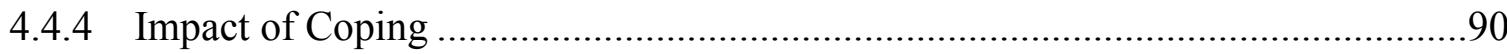

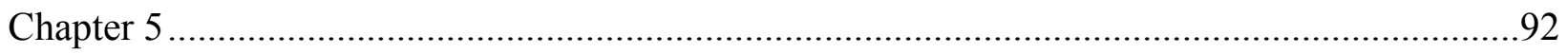

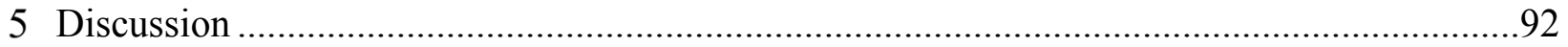

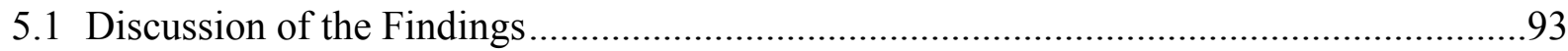

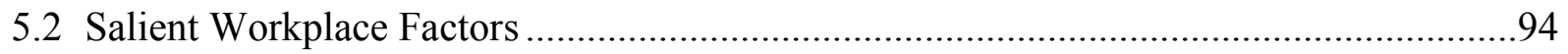

5.2.1 Disclosing a Disability in the Workplace …………...............................................94

5.2.2 My Experience with Asking for Workplace Adjustments ... Error! Bookmark not defined.

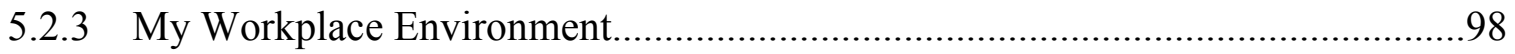

5.2.4 Challenges with my coworkers and supervisors ..................................................100

5.2.5 My Interests and Preferences Align with My Work .............................................101

5.2.6 Personal Strategies to Prevent Future Stress Occurrences... Error! Bookmark not defined.

5.3 Stress and/or Anxiety Inducing Situations in the Workplace .........................................103

5.3.1 ASD-related Stress and/or Anxiety Inducers: The Impact of My ASD

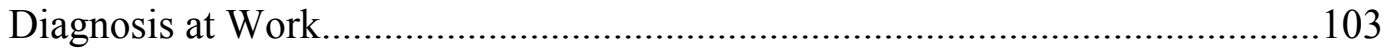

5.3.2 ASD-related Stress and/or Anxiety Inducers: Challenges When I Communicate in the Workplace ...............................................................................104

5.3.3 ASD-related Stress and/or Anxiety Inducers: Tasks at Work ..............................105

5.3.4 My Past Traumas/Experiences Impact My Ability to Work ................................106

5.3.5 My Stressful Experience with Disclosing a Disability at Work ...........................107

5.3.6 How COVID-19 Affected My Employment Experiences …………………….....107

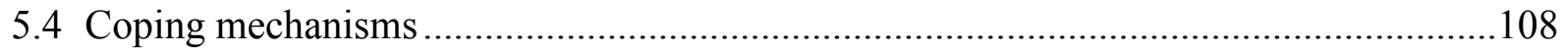

5.4.1 Problem-Focused Coping and Emotion-Focused Coping Strategies ....................108

5.4.2 Coping Strategies in Relation to the Transactional Model of Stress and Coping:

5.4.3 Pushing Through in the Workplace ................................................................111

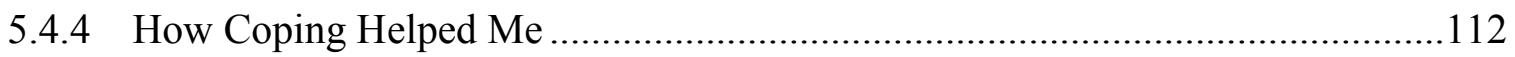




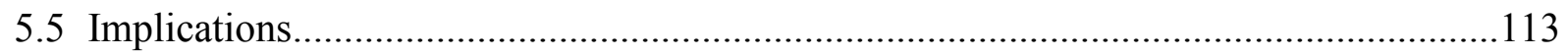

5.5.1 Implications for Employers............................................................................113

5.5.2 Implications for Coworkers and Colleagues......................................................115

5.5.3 Implications for People with ASD ....................................................................117

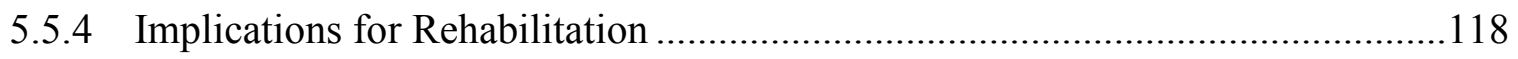

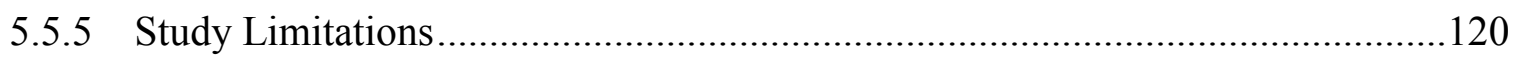

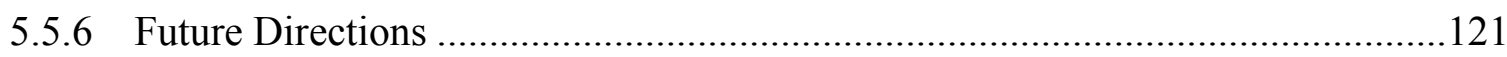

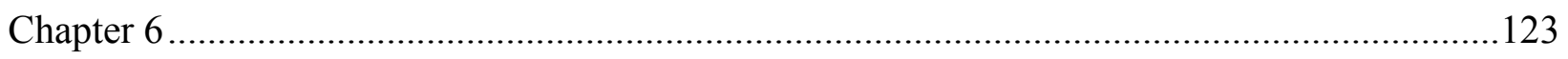

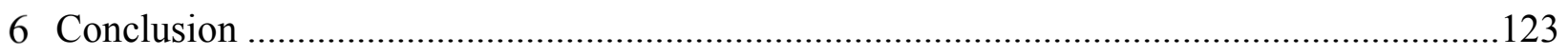

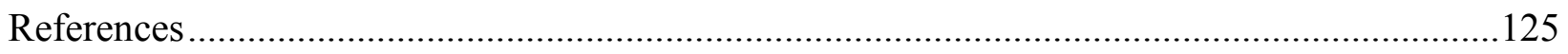

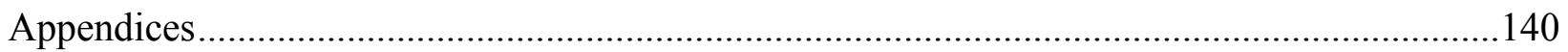

Appendix A:

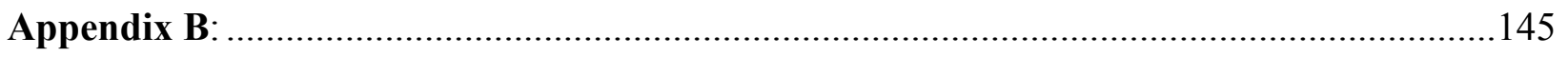

Appendix C:

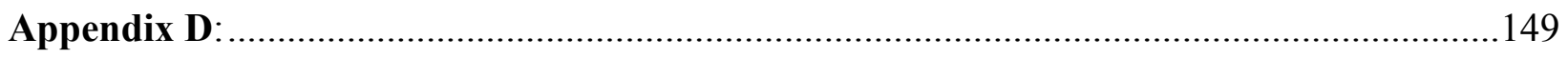

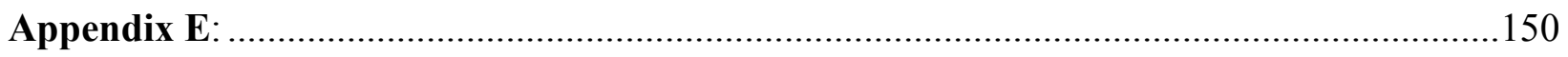

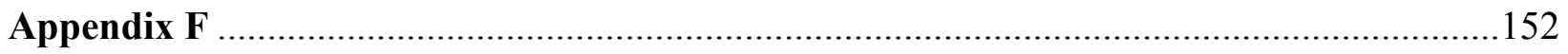




\section{List of Tables}

Table 1: Participant Characteristics ................................................................................ 52

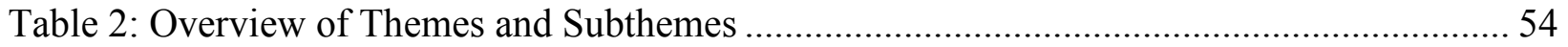




\section{List of Figures}

Figure 1: Relationships between the Identified Themes and Subthemes ................................ 53 


\section{List of Appendices}

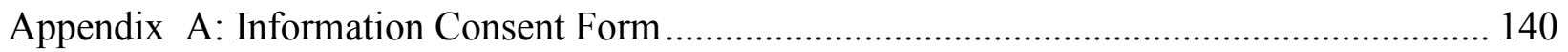

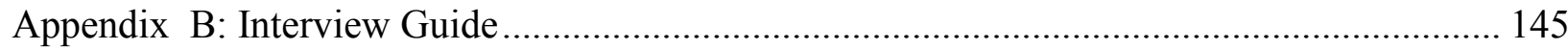

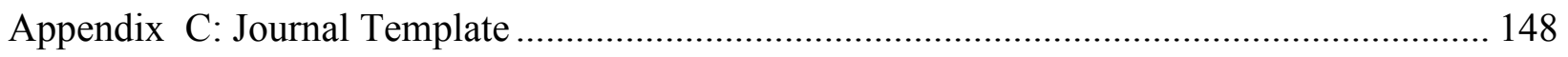

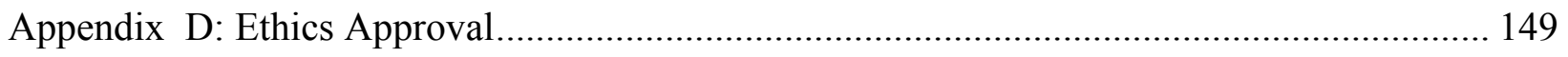

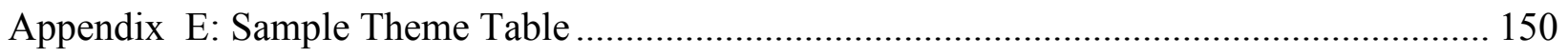

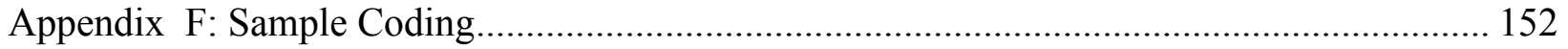




\section{Chapter 1}

\section{Introduction}

\subsection{Brief Background:}

Employment is an important activity for many people because it offers social, financial, and psychological rewards, which can help individuals contribute financially to the economy. Despite the various advantages of employment, participation in work by vulnerable groups can be detrimental due to increasing work demands [1]. Young people with autism spectrum disorder (ASD) often experience barriers in securing and retaining work such as difficulties with communication in the workplace [2]. A competitive work environment, whether triggered by external influences (e.g., coworkers) or internal influences (e.g., personal thoughts), can cause stress for employees with ASD. It is important to discuss the emotional and psychological impact that such demands may have on employed youth and young adults with ASD. Given that the employment rate for adults with ASD remains low [3], it is fundamental to uncover strategies used in employment to help alleviate or manage stress and anxiety on the job as these strategies can be factors in employment retention. Therefore, this Master's dissertation explores the impact of coping in the workplace among youth with ASD, especially during stress and anxiety-inducing circumstances that arise on the job. It is important to discuss and understand coping strategies used by these employees in the workplace because these strategies can play an important role in addressing workplace barriers and facilitators, reduce stress and anxiety in the workplace, and ultimately help foster a positive work environment.

\subsection{Terminology:}

To ensure clarity and consistency, I will define key terms that will be used throughout this dissertation.

Anxiety: As per American Psychological Association, anxiety is characterized as an emotion entailing feelings of worried thoughts and tension and physical alterations (e.g., increase in blood pressure) [4]. This definition was chosen as it combines the mental effects of anxiety an individual can encounter when faced with stressful situations. In this thesis, I 
will be focusing on anxiety (i.e., anxiety in response to overwhelming work demands) as opposed to anxiety disorders (i.e., clinically diagnosed anxiety). Anxiety disorder will be defined as the self-reported clinical diagnosis of anxiety by participants in this study.

Autism spectrum disorder (ASD): According to the Diagnostic and Statistical Manual, Fifth Edition (DSM-5), ASD can be characterized by deficits in social interaction and communication and repetitive and restricted patterns of behaviour [5]. ASD is characterized as a "spectrum" disorder because there are large variations in behaviour and characteristics between individuals with ASD. While ASD can be diagnosed at any age, it is known as a developmental disorder because symptoms usually appear in the first two years of an individual's life [6].

Competitive employment: Refers to positions where those who are employed receive no on the job supports/coaches (e.g., assistance on the job) or day services [7]. Specifically, job coaches often have excessive knowledge and skills to build business and community support sources, help facilitate informed choices, offer a variety of individualized support plans, and coordinate and manage all types of on the job assistance [8]. Hence, in this thesis, there is a central focus on competitive employment with no on the job support/coaches. In addition, those who are competitively employed are not currently enrolled in post-secondary educationrelated programs [7]. This definition was chosen because it focuses on individuals with ASD who are working independently and not receiving on the job support, thus emphasizing the individual responsibility of maintaining and securing employment.

Coping: Coping can be defined as the various thoughts and behaviours an individual has in order to manage internal and external demands of circumstances that are appraised as stressful [9]. Coping in this context is not a trait, but instead a process [9]. This definition was chosen because it relates coping to stress and it focuses on the individual's capability of managing stressful situations. Other studies pertaining to young adults with ASD utilize this definition, ultimately focusing on coping behaviours exhibited by young adults $[10,11]$.

Meltdown: Meltdowns can be characterized by involuntary physical and emotional reactions to a circumstance where there is no perceived exit or get away [12]. In this study, 
"meltdown" was a term operationalized by one participant as crying intensely on the job as a result of working in a highly stressful environment. Therefore, "meltdown" was referenced in the results and discussion sections of this thesis in a manner that reflected the participant's conception and usage of the term.

Pushing through: Pushing through is a term that was operationalized in the interviews by participants in this study. "Pushing through" can be characterized by persevering through a situation despite feeling stressed and/or anxious on the job.

Stress: Stress is defined and interpreted differently depending on the context and discipline of research. However, this thesis defines stress according to Lazarus and Folkman's definition [9], "a particular relationship between the person and the environment that is appraised by the person as taxing or exceeding his or her resources and endangering his or her well-being"(p.19). This term was chosen due to its relation to the term coping and how one responds to overwhelming feelings such as stress and anxiety, which is important in one's adjustment to adulthood.

Youth and young adults: In addition, throughout this dissertation, the terms youth and young adults are used to refer to individuals between the ages of 15-30 years. Youth can be defined as individuals between the ages of 15-24 years [13], and young adults can be defined as individuals from the ages of 18-30 years [14]. These definitions were chosen because they classify the age range between youth and young adults, which may be indicative of the types of challenges faced in employment.

\subsection{Rationale:}

Early adulthood is a stage in life typically full of barriers and concerns for youth and young adults. Early adulthood can be defined by transitions in social and economic roles that occur throughout an individual's late teenage and young adult years (i.e., 14-25 years old) $[15,16]$. A primary challenge with transitioning to adulthood includes finding employment, which is also true for people with ASD [7]. ASD is a neurodevelopmental disorder characterized by difficulties in socialization, communication, and restricted/repetitive behaviours [17]. For adults with ASD, securing employment is associated with higher self- 
esteem and better quality of life [18]. However, individuals with ASD among other developmental disabilities are significantly underrepresented in the workplace [19]. Studies in the United States [20], Australia [21], and Canada [22] report high rates of unemployment for youth and young adults with ASD in comparison to other disability groups. Although youth and young adults with ASD can have many employment strengths, such as reliability [23], attention to detail [24], and exceptional visual skills [25], they often have a difficult time successfully securing and maintaining employment [26].

Difficulties with acquiring and sustaining employment for youth and young adults with ASD can be a result of employment-related barriers, which may include communication difficulties in the workplace or employers having prior negative stereotypes of ASD [27]. However, a prominent barrier to gaining and maintaining employment for youth and young adults with ASD includes coping with the social demands at work [28]. These social demands in the workplace include struggling with communication skills, challenges with maintaining eye contact, and issues with developing and maintaining relationships [5]. Responding to social demands can lead to youth and young adults with ASD potentially feeling stressed and anxious on the job $[29,30]$. Such issues can be problematic for people with ASD because higher levels of stress are linked to worse social functioning [31]. Furthermore, it is important to focus on competitive employment in comparison to sheltered workshops (e.g., long-term work-related placements for people with disabilities) or supported employment (e.g., employment with on the job supports) because competitive employment does not involve onthe-job support for people with ASD [26]. Therefore, youth and young adults with ASD are overcoming challenges in employment (e.g., communication difficulties and negative stereotypes about ASD from employers) independently with no on the job support (i.e., job coaches or employment specialists), which may affect job retention.

Employment-related challenges can vary from personal interactions (e.g., trouble navigating social relationships) to disability disclosure and asking for workplace accommodations $[32,33]$. For youth and young adults with ASD, the processes of disclosing a disability and asking for accommodations can be difficult and daunting because they can put an individual in a vulnerable position [34]. Previous research has established that limitations to disclosing a disability in the workplace include the fear of experiencing negative attitudes 
or discrimination from employers $[35,36]$. The challenges and stressors of competitive employment can negatively impact an individual's well-being (e.g., lead to feelings of frustrations and worthlessness) [37]. Furthermore, stressors are a major problem in the workplace because they can lead to youth and young adults with ASD being unable to cope with work demands, which can result in loss of employment [26]. For these reasons, it is important to recognize the existing coping styles employed by youth and young adults with ASD in competitive employment because these coping mechanisms can be used to reduce job-related stress and anxiety. Securing and retaining employment can be far more difficult for adolescents and adults with ASD given the various barriers to employment [3] and limited opportunities for employment [7,38]. Hence, by discovering potential coping strategies, we can better understand whether these coping styles can help maintain employment for youth and young adults with ASD.

Coping strategies are essential, especially in a fast-paced, constantly changing work environment which can bring about stress [29]. Additionally, researchers have shown that by uncovering potential self-care strategies, adults with ASD are more inclined towards selfimprovement related goals (e.g., being more independent and autonomous) regardless of anxiety-related challenges in employment [39]. Thus, with coping mechanisms in place, employment-related goals can be attained for youth and young adults with ASD. Furthermore, research on coping mainly focuses on caregivers of people with ASD [40] and children with ASD [41]. Thus, there has been no detailed investigation on the coping practices and experiences of youth and young adults with ASD, especially in the context of employment. Moreover, youth with ASD are at high risk for unemployment, especially after two years of finishing high school [42]. Despite the importance of employment among youth and young adults with ASD, there remains a paucity of evidence on how employment can be secured and maintained because employment for this age group appears to be scarce [42]. For these reasons, focusing on the experiences of coping for youth and young adults with ASD in employment is extremely fundamental. 


\subsection{Research Question and Objectives:}

\section{Research Question:}

1. How do youth and young adults with ASD cope with stress and anxiety (i.e., non-clinical anxiety) in competitive employment?

\section{Objectives:}

1. To understand the subjective feelings of stress and anxiety that youth and young adults with ASD experience within competitive employment.

2. To understand subjective coping strategies used by youth and young adults with ASD when faced with work-related stress or anxiety.

\subsection{Importance of this study and gaps addressed:}

Focusing on the various perspectives on stress, anxiety, and coping in the workplace can help in the development and refinement of vocational programs and transition policies during employment preparation for youth with ASD. Much literature focuses on pre-employment and/or work readiness training for youth and young adults with ASD [33]. So far, however, there has been little discussion about the personal experiences with stress and anxiety in the workplace. This research aims to understand the experiences of employment and gain insight into how youth and young adults with ASD maintain their employment status. For those who are employed and have not yet developed coping strategies in the workplace, this research can help provide further insight and knowledge on how stress and anxiety-provoking circumstances are managed among some youth and young adults with ASD. This study provides an important opportunity to advance the understanding of employment-related challenges among youth and young adults with ASD, specifically stress and anxiety, and has potential to point to factors related to employment retention among this population.

Furthermore, the findings from this thesis have potential to help inform employers of youth and young adults with ASD of these potential stressors in the workplace, which can lead to better support and inclusion on the job. Hence, if these experiences are known to employers, it could help facilitate an inclusive work environment for youth and young adults with ASD and provide insights into the challenges and barriers employees with ASD experience in an employment setting. Therefore, these lived experiences also aim to provide awareness for employers, which is important especially in an inclusive employment environment. 
To date, the majority of literature on employment outcomes for youth and young adults with ASD focus on factors related to securing employment [43]. The existing literature on youth and young adults' experiences with ASD in the workplace highlight the challenges and barriers in securing an employment position [43]. In relation to the existing literature on employment outcomes, personal experiences with employment that discuss barriers and facilitators to successful maintenance of employment are limited in the literature [29,44]. Hence, the nature of how youth and young adults maintain employment remains unclear. Exploring the direct perspectives and experiences of employment among youth and young adults with ASD can help uncover the potential stress and anxiety-triggering situations (i.e., barriers to employment) that arise on the job, and how coping strategies (i.e., facilitators to employment) help overcome these challenges.

The mechanisms that underpin the processes of disability disclosure and asking for workplace accommodations are not fully understood in the literature. There is limited research focusing on the personal experiences with the processes of both disability disclosure and workplace accommodations for youth and young adults with ASD [45]. In the literature, disclosure rates vary by age, inter-generational differences, and comfort level, while more research is required to explore how the disclosure process varies by industry type [33]. While there are benefits to disclosing a disability in the workplace (e.g., allows direct communication between employers and employees, allows for reasonable accommodations), there are also limitations (e.g., stigma and discrimination from employers) [44]. Due to the varying experiences with disability disclosure, studies indicate the importance of knowing the conditions for disclosure and understanding workplace experiences once people with ASD have been employed for a certain period of time because disclosure can vary depending on how long an individual has been employed $[33,44]$. The existing literature on accommodations for young adults highlights the importance of providing environment modifications, customized workplace interventions, and peer mentorship [22,46,47]. This indicates a need to understand the various personal experiences and perceptions of disability disclosure and requesting for accommodations that exist among youth and young adults with ASD. 
Focusing on youth and young adults with ASD is important because these age groups face several barriers in securing employment, especially related to social functioning, which can ultimately interfere with employment experiences [45]. Finally, when employment experiences are discussed in the literature in relation to youth and young adults with ASD, barriers and facilitators to employment are highlighted without a specific focus on workrelated stress and anxiety [32]. For youth and young adults with ASD in employment, stress and anxiety on the job can be reasons as to why employment is not successfully retained. Thus, highlighting the impact of stress and anxiety is critical as it can further uncover coping mechanisms that may help maintain employment. 


\section{Chapter 2}

\section{Literature Review}

\subsection{Defining Autism}

ASD is a developmental disorder that is often associated with difficulties in communication and social skills [48], repetitive behaviours [48], discomfort with changes in schedules [28], troubles with expressing emotions [28], adapting to new tasks and roles [28], and sensory differences (e.g., sounds and smells). Difficulties in communication and social skills may include impairments in social reciprocity, maintaining and understanding relationships, and non-verbal communication [48]. In addition, there is a wide variation in the type and severity of symptoms that people with ASD experience [6]. Other social communication behaviours can include: reduced eye contact, having difficulties maintaining a conversation, having facial expressions that do not correspond to what is being said, and having an unusual tone of voice [6]. In regard to restricted/repetitive behaviours, this may include: repeating certain behaviours (e.g., repeating words continuously), experiencing distress with changes in routine, and being hypersensitive or hyposensitive to sensory input such as noise, light, temperature, and clothing [5,6]. For instance, many individuals with ASD are hyper-sensitive to loud noises in their environment [49].

According to the Centres for Disease Control and Prevention, ASD is diagnosed in every 1 of 59 children [50]. A recent meta-analysis shows that male: female prevalence ratio is 4.56:1 in studies that detect cases from existing medical or educational records, however in studies that actively detect cases in the general population, the prevalence ratio is $3.25: 1$ [51]. This finding highlights the current under-recognition of ASD in females because in comparison to males, females are more likely to be diagnosed with ASD later in their life [52]. Several lines of evidence demonstrate that the threshold for ASD diagnosis is higher for females, requiring more emotional, behavioural, and cognitive challenges to be recognized $[53-55]$.

In previous versions of Diagnostic and Statistical of Manual of Mental Disorders manual (DSM IV-DSM-IV-TR), individuals were diagnosed with one of several related conditions, including autistic disorder, Asperger Syndrome, and/or pervasive developmental disorder not 
otherwise specified (PDD-NOS) [5,6]. Although these diagnoses were considered related, they were not conceptualized as a spectrum within these criteria. However, the manual was modified in 2013 (DSM-5) and changed the diagnosis of ASD; these separate diagnoses have now been combined into one diagnosis named "autism spectrum disorder" $[5,6]$. Thus, prior to 2013, people with ASD may have been diagnosed with either AD, AS or PDD-NOS. Going forward, if studies differentiate between the now-revised classification of ASD (i.e., instead using AS or PDD-NOS), I will proceed with the classification given by the original studies and not change their classifications. In addition, since modifications to the diagnostic criteria were in 2013, some participants in this study would have been diagnosed with the earlier diagnostic criteria (i.e., DSM-IV-TR). As such, some participants are therefore more likely to identify in this way (see Table 1).

\subsection{Employment for Youth and Young Adults with ASD}

In this section of my thesis, I discuss employment for youth and young adults with ASD. More specifically, I discuss employment rates for youth and young adults with ASD followed by a section exploring employment-related skills that youth and young adults with ASD offer to a work environment. Employment rates for youth and young adults with ASD are of interest because these rates can point to the barriers and facilitators to successful employment as reported in the literature. Furthermore, this leads to a discussion of employment-related skills, which is an important aspect of employment for youth and young adults with ASD because these skills highlight the strengths that youth and young adults with ASD offer to employment. I then discuss the process of finding employment, which touches on disability disclosure and accommodations, barriers to employment and then conclude by discussing the positive experiences with employment. More specifically, in the last few subsections of employment, I discuss the process of finding employment, which highlights the challenges in securing and maintaining employment. I then conclude with the benefits of hiring inclusively and recount positive workplace experiences of employees with ASD as reported in the literature.

\subsubsection{Employment Rates for People with ASD}

Employment provides a source of independence that goes beyond economic gains, offering a sense of achievement, and leading to opportunities for socialization [23]. For adults 
with ASD, the motivation to get employed is no different from adults without a disability [23]. Even when the overall job market was booming, statistics on employment opportunities for youth and adults with ASD portrayed a gloomier image [3]. For young adults with ASD in Canada, they are more likely to be unemployed than their peers with other disabilities [56]. In Canada, the employment rate among adults with ASD is merely $22 \%$ compared to $74 \%$ amongst Canadians without disabilities [22]. The 2012 Statistics Canada report divided the disability groups into 11 subtypes with associated employment rates: seeing (37.6\%), hearing (47.9\%), mobility (36.0\%), flexibility (39.1\%), dexterity $(31.7 \%)$, pain $(46.0 \%)$, learning (28.8\%), memory (30.7\%), developmental (22.3\%), mental and/or psychological (35.9\%), or unknown disability type (61.0\%). Hence, in comparison to the various disability subgroups in Canada, individuals with developmental disabilities (e.g., ASD) had the lowest employment rate.

In a recent Ontario study of 480 youth and adults (ages 16 and over) with ASD, 13.9\% of participants aged 20 and over relied on full-time employment for their primary source of income and $6.1 \%$ of this sample relied on part-time employment [57]. Furthermore, 209 out of the $480(58.2 \%)$ sample relied on the Ontario Disability Support Program (ODSP) as a main source of income [57]. According to the Canadian Community Health Survey: Annual Component in 2017, 33\% of adults with ASD aged 20-64 were employed in comparison to $79 \%$ of adults without disabilities [58]. In a study that investigated employment rates for adults with developmental disabilities, Zwicker et al. reported that $21.5 \%$ of youth and adults aged 15-64 with ASD were engaged in the labour force (i.e., employed or looking for employment) [59]. The evidence presented in this section suggests that the employment rate for youth and young adults with ASD has been and continues to remain low in comparison to other disability groups and those without a disability.

\subsubsection{Employment Skills}

Youth and young adults with ASD can have many employment strengths and skills including increased visual search abilities and a systematic type of work style with skills such as attention to detail [30]. In addition, individuals with ASD tend to show a higher degree of reliability and dependability, are often more willing to do repetitive work, and have the ability to develop a high level of skills in specialized and narrow areas of work (e.g., software 
testing) $[21,23,60]$. The literature on employment for adults with ASD highlighted types of employment that adults with ASD tend to be clustered into, many of which require precise accuracy, information processing, high degree of technical abilities, and high levels of focus for long periods of time (e.g., office and administrative-related occupations) [20,21,33,61]. These are just a few of the skills described in the literature, however it must be noted that these skills may not be applicable to every individual with ASD. Due to the heterogeneity of ASD, there may be strengths to employment that have not been highlighted or described in the literature. Thus, youth and young adults with ASD without these aforementioned strengths also deserve options for employment. In addition, is important to consider each individual's strengths and potential contributions to the realm of employment.

Despite various strengths and skills, adults with ASD often experience high rates of unemployment, have difficulty adjusting to new employment environments, and are less likely to be employed in comparison to adults without disabilities [62-64]. Furthermore, there is a high prevalence of adults with ASD who are over-educated for their current job [21]. Together, these findings indicate the importance of focusing on youth and young adults with ASD because even with higher qualifications, youth and young adults with ASD are found in positions for which they are overqualified [44].

\subsubsection{Finding Employment, Disability Disclosure, and Accommodations in the Workplace}

The process of finding employment can be difficult for youth and young adults with ASD [1]. Specifically, ASD-related challenges (e.g., difficulties with navigating social situations) can make it difficult to obtain employment opportunities [1]. For instance, in an investigation into the experiences of employment among young adults with ASD (mean age of 22.9 years), Anderson, Butt, and Sarsony found the job interview process to be a prominent barrier to securing employment [32]. Specifically, some young adults with ASD described their

experience with the process of job interviews as vulnerable and threatening, and a reason as to why a job was not successfully attained [32]. For these young adults, the interview process was daunting because of their personal fears of potential judgement and discrimination from their employers due to their disability [32]. In an employment setting, people with ASD may speak informally or use an inappropriate tone or volume when speaking with coworkers or 
employers [33]. Navigating social interactions are often difficult even before employment is attained and it often begins with conquering the interview process [28].

For other young adults with ASD, the question of whether to disclose or not to disclose their diagnosis in an interview is another barrier to finding employment [32]. Some young adults with ASD report that disclosing a disability in an interview can possibly lead to accommodations and acceptance in the workplace, whereas others recommend against disability disclosure due to potential fear of discrimination [32]. Several studies described employer concerns associated with hiring people with a disability, which included increased costs for constant supervision, training and accommodations in the workplace [65-67]. However, one study found that employing adults with ASD can provide employers and organizations with benefits (e.g., detail-oriented in their work ethics) without acquiring additional costs [23]. Employers may also be cautious of increased costs related to occupational modifications or workplace accommodations for adults with ASD, however one study found that these costs are relatively minor in comparison to modifications needed for adults with a sensory or physical disability [68]. Despite the lower cost of accommodations, it is important to note that people with disabilities deserve accommodations from their employer, regardless of the type of disability they have. The literature may indicate varying levels of cost associated with accommodating individuals with different disabilities, but employers need to recognize and acknowledge that disabilities are heterogeneous and individual experiences and limitations are important factors to understand when making accommodation-related decisions in the workplace.

Since it is vital for employers to understand and recognize individual needs in the workplace, it is important to understand the context as to why workplace accommodations can be valuable for individuals with ASD. Workplace accommodations can be beneficial for youth and young adults with ASD because challenges on the job can be mitigated by various types of employment-related accommodations [69]. Some accommodations that help adults with ASD maintain their employment include technology-based assistive devices (e.g., Apple iPod) [70]. Such devices could be used for task reminders and behavioural self-management adaptations [70], audio coaching [71], and reducing the need of personal support and job coaching [70]. Other workplace accommodations consist of reducing environmental triggers for youth and young adults with ASD, which may include reducing distractions from 
coworkers and/or crowding, managing noise levels, and using suitable lighting [21,62,72]. Without the aid of accommodations, navigating work demands can be difficult as there are a number of barriers that prevent youth and young adults with ASD from being successful in a competitive work environment [69].

\subsubsection{Barriers to Employment}

Youth and young adults with ASD often face barriers when seeking and retaining employment. Therefore, this portion of my thesis addresses the potential barriers to securing and maintaining employment. This section includes two subsections: ASD-related barriers to employment and employers/societal challenges that impact employment opportunities. ASDrelated barriers to employment include social communication difficulties and behavioral challenges whereas employers' and societal challenges include disability disclosure and employer's attitudes and stigmas toward ASD.

\subsubsection{ASD-related barriers to employment: Social Communication Difficulties}

There are a number of factors that contribute to underemployment and/or limited employment opportunities for youth and young adults with ASD [2]. Much of the current literature on employment-related challenges among youth and young adults with ASD focus on communication difficulties as a barrier to successful employment for youth and young adults with ASD $[2,73,74]$. Communication difficulties can pose a threat to youth and young adults with ASD because these social challenges can lead to poor employment outcomes (e.g., job loss) [73]. Difficulties with communication can pose a threat from the employee perspective as well. For example, in a comprehensive study of employment experiences among adults with ASD, Hurlbutt and Chalmers found several participants blaming their difficulties with employment on communication issues within an employment environment [18]. Specifically, this included speaking too bluntly, fixating on things that a coworker says, and not being able to get clarification from their boss [18]. Thus, the study found that a lack of communication skills can interfere with an individual's employment status [18]. For several adults with ASD, the challenge with understanding social cues and initiating social communication can lead to fewer social interactions [75]. These challenges with social communication can lead to anxiety and further withdrawal from social situations [60]. 
Challenges with social communication can transfer over to employment as adults with ASD might have difficulties conveying their thoughts in an appropriate manner, ask questions too frequently, and have trouble managing coworkers' interactions [18]. One study that looked at factors associated with successful transition to work for adults with ASD (ages 19-29 years) found that social communication difficulties (e.g., speaking too bluntly) can be perceived as rude and offensive by coworkers at work [46]. Similarly, in a brief report that was set out to explore employment among adults with ASD, Burt, Fuller and Lewis noted that poor social skills such as interrupting conversations, inability to communicate needs to employers, and the incorrect usage of language phrases in the workplace contributed to the inability to retain employment [76].

In many cases, the blame is often placed on the individual with ASD for not acclimatizing to the workplace environment or possessing social skills that are atypical. The blame can be shifted away from individuals with ASD if employers are aware of disability inclusion in the workplace, which is likely to reinforce their hiring choices [77]. However, social deficits can limit opportunities for youth and young adults with ASD seeking employment. Addressing social deficits is necessary because social abilities directly affect employment success of adults with ASD $[28,73]$. Furthermore, it is apparent that social communication difficulties can severely impact the overall employment experience for individuals with ASD because a large proportion of workplaces require adherence to social norms and rules [78].

\subsubsection{ASD-related barriers to employment: Behavioural Challenges}

Another barrier to employment includes behavioural challenges [2]. For individuals with ASD, ritualized behaviours may also manifest in the workplace through strict adherence to routine, extreme resistance to change or with unprecedented events, inflexibility in thinking, and through continuous questioning or speaking on the same topic [79]. These aforementioned behaviours may pose as challenges in the employment environment for some youth and young adults with ASD. The literature indicates that $30 \%$ to $50 \%$ of youth and young adults with ASD show maladaptive behaviours $[7,80]$, which can negatively affect dayto-day functioning and may include behaviours such as aggression, self-injury, and uncooperative behaviours [7]. Any form of these aforementioned behaviours can potentially 
discourage employers from hiring or retaining employees with ASD [2,7]. Maladaptive behaviours are often not well tolerated in a job setting because they can prevent employment and lead to segregation in the workplace [81]. For instance, in a study that investigated participation in the workplace for adults (average 23.83 years of age) with ASD over the course of 12 years, findings suggested that behavioural challenges influence the type of employment activities over time [43]. To elaborate, those with fewer maladaptive behaviours were more likely to participate in employment [7,43]. In a similar manner, Smith and Coleman in their investigation of understanding the effects of maladaptive behaviour on employment for three young adults with ASD (ages 26-28 years) found that behavioural challenges (e.g., tantrums) negatively impacted employment retention [82]. Maladaptive behaviours related to ASD can be associated with stress, clinically diagnosed anxiety disorders, and other comorbid psychiatric conditions $[83,84]$. Therefore, behavioural challenges in the workplace can be a barrier to securing or maintaining employment [2].

\subsubsection{Employers/Societal Challenges: Disability Disclosure}

Disability disclosure, which refers to the process of disclosing a disability in the workplace, is often a barrier to employment for adults with ASD [44]. Although disclosure can be advantageous for some adults with ASD because it allows for accessing job accommodations [18], there is often stigma associated with disclosing a disability $[85,86]$. In one study, adults with ASD with varying employment experiences reported being hesitant in disclosing their disability because of fear of not being hired or employers using their diagnosis of ASD as an excuse to terminate employment [18]. Similarly, disclosing a disability can limit employment opportunities due to discriminatory practices on the job once an applicant is hired $[87,88]$. For instance, in a study that explored the stigma associated with ASD in the workforce, Johnson and Joshi found that some adults with ASD preferred nondisclosure in the workplace due to feelings of discrimination and stigma from employers [88]. Detailed exploration of employers' perspectives of including youth and young adults in the workplace by Lindsay et al. showed that some employers have unintentional discriminating attitudes towards employees with disabilities [45]. Hence, stigma may present itself in an employment setting, which can deter successful employment for individuals with ASD [88]. For these reasons, it is important to further explore the disability disclosure process to see whether experiences with this process can be reflected positively or negatively by youth and 
young adults with ASD. By doing so, it can help identify whether this process is an important factor for successful employment.

Some studies also reported positive experiences with disability disclosure. In a study conducted by Romualdez et al., they found that in a sample of 238 adults with ASD with employment experiences, over a third of participants felt that disclosing their disability was a positive experience [89]. Other studies found that disability disclosure provided benefits for employees with ASD such as greater acceptance in the workplace and increased awareness and advocacy of ASD $[22,33,90]$. The process of disability disclosure may be positive for some employees with ASD, but for others, it remains a barrier in the workplace. As such, the process of disability disclosure can be daunting for many youth and young adults with ASD, it can pose as a barrier to securing and retaining employment, and it requires further investigation on the varying experiences.

\subsubsection{Employers/Societal Challenges: Employers' Attitudes}

Another barrier to employment stems from potential employers and their perceptions about hiring and retaining employees with ASD [3]. In some situations, employers have limited experience with hiring people with disabilities, especially individuals with ASD, which can contribute to biases, misconceptions and potential discrimination [91]. For instance, Morgan and Alexander found that employers mentioned concerns related to increased cost, lack of productivity, and noticeable skill deficits when hiring individuals with ASD [92]. Additionally, another study found that potential employers stated concerns related to attendance, safety, and social skills when deciding whether to hire individuals with developmental disabilities [93]. Scott et al. reported that employers rated 1 in 5 job applicants with ASD as showing significant challenges in social skills in the workplace, which contributed to employers being unwilling to hire adults with ASD [23]. Whether these behaviours implicitly suggest to employers that job applicants with ASD may have unrelated deficits to areas such as intellect or cognition, or because employers believe that normative social behaviour is imperative to the job role itself, or even because employers sense that atypical social behaviours may bother colleagues, these point to potential reasons as to why employers hesitate to hire inclusively [3]. Although there may be challenges in employing adults with ASD (e.g., difficulties following instructions or inflexibility in work 
schedule/routine) [94], most of these barriers can be reduced by employers adapting work tasks, having direct communication with employees, and understanding individual support needs $[23,61,69,72]$. One systematic literature review on employer attitudes towards workers with disabilities found favourable employer attitudes with hiring inclusively when organizations were larger (greater than 100 employees) and if employers had previous experience with hiring inclusively in the workplace [91]. The reason for large organizations hiring more inclusively may be attributable to having more resources and less concern for costs related to supervision, training and accommodations [91]. In addition, previous experience with working with individuals with ASD can encourage employers to hire inclusively [23]. To help lessen or eliminate these biases and misconceptions, it may be beneficial to have employers be informed of the experiences of employment from the perspective of employees with ASD. By being informed of these perspectives, employers may understand the importance and impact of inclusive hiring and disability awareness in the workplace.

\subsubsection{Benefits of Hiring Inclusively}

Employers also note positive experiences with employing individuals with ASD [23,95]. For instance, employers indicate the positive impact of employing adults with ASD including the addition of new and innovative skills, increase in ASD awareness and a positive outlook on workplace inclusion [23]. Cimera and Burgess found that employees with ASD benefit employers by working consistent hours every week for a significant amount of time, which demonstrated reliability [95]. Other advantages that employers identify with hiring individuals with ASD include regular attendance, positive coworker relationships, long-term tenure, greater work efficiency, and awareness and education related to disabilities [92,96]. Being more informed and aware of ASD can help employers understand the importance of job fit and larger appreciation for the impact of inclusive employment including workplace morale and quality of work [97]. Employers also note that hiring individuals with ASD helps promote inclusive attitudes and greater intentionality in relation to inclusive hiring [97].

\subsubsection{Positive Experiences with Employment Among People with ASD}

Despite negative experiences with employment for youth and young adults with ASD, there are a number of positive employment experiences as well $[21,85,98]$. For example, one 
study reported the positive experiences of employment from a national survey of Australian adults with AS and High Functioning Autism, which divided the positive experiences into six different themes [21]. These themes included self-actualization, social and collegial factors, job roles and work content, and pay and benefits. Under the theme of self-actualization, participants reported a positive work experience due to having the opportunity to apply and develop skills and knowledge, having the freedom to be independent, and being accepted and valued. For social and collegial factors, participants mentioned positive relationships with colleagues and enjoyable interactions with customers. In regard to job roles and work content, participants often reported enjoying job roles and work-related tasks with favourable working conditions. The last theme is pay and benefits and positive experiences, which included financial benefits such as earning money and perks on the job (e.g., leave allowance) [21]. Other positive experiences with employment can relate to one's personal interest and ability on the job. For example, in an investigation into the experiences of daily life for younger and older adults with AS, Smith and Sharp found adults who were aware of their strengths and weaknesses interacted more with employers, which boosted their confidence and selfacceptance [98]. In Krieger et al.'s study of adults with AS (ages 30-45 years) in employment, participants described that having work experience helped them learn more about themselves and lead to finding satisfaction in work [85]. Having self-determination and belief in one's skills can lead to positive experiences in employment as well [85,98]. Taken together, these studies provide evidence that positive experiences with employment exist despite the barriers to secure and maintain employment for youth and young adults with ASD.

\subsubsection{Conclusion}

Employment for youth and young adults with ASD is a complex process. The literature reports several positive experiences with employment, however, there are quite a few barriers to maintain, let alone, secure employment. Together, these studies provide important perspectives into the barriers to secure employment, a common barrier being difficulties with social communication for youth and young adults with ASD [2,73]. However, there remain several aspects of employment about which relatively little is known. In view of all that has been mentioned so far, one may suppose that there is a critical need to focus on how youth and young adults maintain employment despite barriers in the workplace. For youth and young adults with ASD, maintaining employment can be difficult with no on-the-job support. 
In many cases, individuals with ASD who are employed must cope with work-related challenges, such as communication difficulties and asking for accommodations from their employers independently, which can be an intimidating process. Hence, it is important to focus on the personal struggles of stress and anxiety that individuals experience in competitive employment because individuals are securing and maintaining employment independently with no on the job support. Gaining insight on personal experiences with challenges and stressors in employment for youth and young adults with ASD can address this important gap in literature. Poor outcomes in employment can negatively influence an individual's socioeconomic status, standard of living, and most importantly mental health [99]. Thus, it is vital to recognize the perspectives of these employees because it leads to better understanding and further awareness of challenges faced in the workplace.

\subsection{Stress and Anxiety in the Workplace}

In the second section of my thesis, I discuss stress and anxiety (i.e., non-clinical anxiety) for youth and young adults with ASD. More specifically, I discuss the impact of stress, which includes a discussion on how youth and young adults with ASD experience and respond to stress. In the same vein, I discuss anxiety among youth and young adults with ASD and its associated impact. I then discuss how stress and anxiety is experienced in the workplace for youth and young adults with ASD. It is essential to discuss stress in relation to youth and young adults with ASD to determine how stress affects different parts of their lives, and equally important, how they respond to stress. Stress is of key interest because this discussion can open opportunities to uncover the impact of stress, specific to the workplace, for youth and young adults with ASD. Similarly, is also important to look at the relationship between anxiety and ASD, which can eventually lead to a discussion on anxiety and its influence in the workplace.

\subsubsection{Stress for Youth and Young Adults with ASD}

Compared to the general public, individuals with ASD experience higher levels of general stress and anxiety [39]. In the literature, stress and anxiety levels appear to be heightened for both children and young adults with ASD $[39,100]$. In an investigation of depression and anxiety among adults (ages 16-62) with ASD, Nah et al. reported that adults with ASD are characterized by higher levels of anxiety, depression, and stress in comparison 
to adults without ASD [100]. Stress is of key interest because it can vary from individual to individual and the corresponding response to stress can differ as well [101]. In a randomized control trial comparing stress in youth and adults with ASD (ages 16-45) with youth and adults without ASD, Bishop-Fitzpatrick et al. reported that youth and adults with ASD experience significantly higher levels of stress than adults without ASD, which contributed to the predictions of social functioning in everyday life [31]. More specifically, for adults with ASD, greater stress was associated with worse social functioning, which underscores the impact of stress on social outcomes[31]. In the same vein, Hirvikoski and Blomqvist in their study found adults with ASD reporting higher levels of subjective stress and a perception of lower stress management ability in relation to everyday life stressors [102]. In addition, Hirvikoski and Blomqvist found that core features of ASD (i.e., challenges with schedule changes) were associated with distress because symptoms could bring about stressful circumstances in everyday life for adults with ASD [102]. Despite high levels of perceived stress for ASD, Bishop-Fitzpatrick et al. found adults with ASD had poor stress management/coping strategies to combat these stressors in everyday life [31]. Among youth with ASD, in a study that explored the challenges of becoming an adult, Cheak-Zamora, Teti and Maurer-Batjer found that youth with ASD (ages 18-25) felt stressed when engaging in conversations with peers due to not understanding the rules of social etiquette [103]. Similarly, youth reported feeling stressed when learning new skills (e.g., developing social skills in conversation), taking on new responsibilities (e.g., finding employment), and developing and maintaining friendships [103]. Therefore, by having stress impact many aspects of everyday life, it can negatively affect individuals maintaining their employment status [30].

\subsubsection{Anxiety for Youth and Young Adults with ASD}

Studies indicate that youth and young adults with ASD are more likely to suffer from high levels of anxiety than those without ASD [10,32,104]. To elaborate, even in the earliest descriptions of ASD, Kanner found that characteristics of ASD including insistence on sameness, repeated/fixed behaviours, and routines seemed to have a relationship with anxiety [105]. Anxiety is highly common among youth with ASD and in many cases, there may be overlap between anxiety and ASD symptoms [106]. For instance, youth with ASD may experience higher levels of anxiety related to changes in their environment or routine 
$[107,108]$. In a similar manner, youth may be anxious when barriers or obstacles prevent them from engaging in compulsion or stereotyped behaviour [109]. Despite the frequent presentations of anxiety, anxiety is not generally considered a core characteristic of ASD since not all youth with ASD show this symptom [106].

Youth with ASD experience a variety of anxiety disorders, with the most common anxiety disorders being specific phobias, social anxiety disorders, generalized anxiety disorder, and obsessive compulsive disorder [110,111]. In the same vein, Gillot and Standens' study of anxiety in adults with ASD found that obsessive behaviours were associated with increased anxiety levels [112]. In the same manner, another study found that in a sample of 54 participants with ASD or AS, anxiety increased as children and youth with ASD or AS grew older in comparison to children and youth without disabilities [110]. For youth with ASD, typical fears may include fear of the dark, needles and injection, insects, and in many cases, the experiences of these fears are no different to typically developing youth [110].

In addition, with reported challenges in social communication, youth with ASD often experience higher levels of social anxiety as they may become overwhelmed by social interactions [106]. Thus, these findings suggest that youth and young adults with ASD may experience some type of anxiety, which may negatively impact mental health and overall quality of life [113,114].

\subsubsection{Stress and Anxiety in the Workplace}

The responsibilities of being employed can lead to negative emotional effects on individuals as studies point to stress being a major obstacle in the workplace $[30,115]$. In comparison to neurotypical adults, adults with ASD experience higher levels of stress in general [31]. In addition, what is perceived as a stressor can differ from individual to individual [102]. For some youth and young adults with ASD, interactions with others can be stress inducing [29], while for others, unanticipated schedule changes at work can induce stress [116]. Likewise, responses to stress can differ from individual to individual depending on the context and the problem at hand. For instance, Hayward, McVilly and Stokes reported that in a sample of 76 adults (44 with autism and 32 neurotypical adults), several adults with ASD felt stressed from interactions with other coworkers and instead preferred to work alone [29]. 
For many participants, stress stems from interactions with others, which is reflected through another study that explored the lived experiences of women with ASD (ages 18 years and over) from an online autism community web page [31]. Women from this study expressed their desire to secure and maintain employment, but then explained that symptoms related to ASD (e.g., social deficits) contributed to work-related stress [31]. This view was supported by Mcknight-Lizotte who found adults with AS reported higher levels of stress in the workplace because they found it difficult to work with neurotypical coworkers [73]. McKnight-Lizotte's study illustrated these difficulties as participants with AS reported on the stress of not understanding the social rules of the environment, not knowing what topics are appropriate to discuss, and having difficulties asking for help [73]. For other youth and young adults with ASD, the constant need to perform well and conquer barriers to employment can lead to maladaptive behaviours such as outbursts [62]. Likewise, adult employees with ASD often want to be productive and meet the employer's expectations, which can also lead to stress in the workplace [30].

The workplace environment can also constitute stress for youth and young adults with ASD and can have a significant impact on successful employment $[69,92]$. The physical workplace environment including sensory characteristics are a major barrier for adults with ASD [94]. This view was supported by Pfeiffer et al. who investigated the relationship between sensory aspects in a work environment and job satisfaction from the perspective of adults with ASD, found that individuals with sensory sensitivity in the workplace were significantly less satisfied with their workplace position [117]. To elaborate, an individual with high levels of sensitivity in a noisy and loud environment tend to be more easily distracted or may find the environment distressing or uncomfortable [117].

In comparison to people without disabilities, youth and young adults with ASD often experience higher levels of stress and anxiety and lower coping ability [102]. Therefore, given that youth and young adults are more prone to stress and anxiety in an employment setting, feelings of stress and anxiety can be heightened on the job [31,102]. These studies on stress in ASD suggests that impaired stress management mechanisms may be inherent in the symptomatology of ASD, which may explain why adults with ASD experience higher levels of stress [31]. 
Furthermore, vulnerability to stress can lower an individual's ability to manage the social demands in the workplace [29]. An individual's perception of their ability to cope with a stress-related event is essential for how they internalize that stress [118]. Youth and young adults with ASD may have fewer resources (e.g., social support) to cope with interpersonal relationships in the workforce, which inevitably results in stress [29]. More specifically, Hayward, McVilly and Stokes found that adults with ASD in the workplace were more prone to stress because of their challenges with communication, which inevitably made it difficult to ask for help on the job [29]. Therefore, resources to manage stress (e.g., asking for help), which are usually used to decrease stress on the job, can alternatively increase and induce stress for adults with ASD [29]. Consequently, this can ultimately lead to a net loss of resources and result in getting burnt out easily, not being able to handle stress very well, and having difficulties enduring stressful circumstances [29]. Thus, it is critical to understand the way youth and young adults with ASD cope with and respond to stress because it is an important adaptation in adulthood, which can impact one's mental wellbeing [31]. In addition, it is important to understand the unique individual characteristics in relation to the workplace environment to ensure successful employment for youth and young adults with ASD [117]. Hence, this highlights the importance of uncovering and understanding individual experiences to stress, especially in a changing work environment.

Similar to experiences with stress in the workplace, when adults with ASD encounter difficulties in the workplace and are without employment supports, their levels of anxiety increase tremendously [18,119]. Therefore, this indicates the impact of anxiety, especially when there are no on-the-job supports for youth and young adults with ASD. Reasons for increased levels of anxiety can vary from individual to individual. For some youth and young adults with ASD, the concept of securing employment is difficult as the nature of the interview process induces higher levels of anxiety [32]. For others, the nature of the workplace environment and working with neurotypical coworkers may induce feelings of anxiety. For instance, Hurlbutt and Chalmer found that people with AS reported challenges with working with people without disabilities, which caused them to experience higher levels of anxiety on the job [18]. Hurlbutt and Chalmer's study also found participants becoming overwhelmed by topics deemed appropriate to discuss in the workplace and often reported not 
knowing when to ask for help [18]. Therefore, overwhelming situations can often lead to stress and anxiety, which are major concerns in the workplace.

Given that anxiety is very common among people with ASD, some symptoms related to ASD (e.g., inability to cope with changes to schedules) prevent youth and young adults from applying coping skills to manage stress and anxiety-provoking circumstances [120]. In employment, stress and anxiety-inducing situations can occur on an everyday basis and youth and young adults with ASD have fewer resources to cope with these employment demands [29]. Therefore, this highlights the importance of developing and employing coping skills in the workplace.

\subsubsection{Conclusion}

Stress and anxiety are major challenges in the workplace for people with ASD. Given the various challenges to employment (e.g., difficulties with social interactions in the workplace), if youth and young adults with ASD utilize coping mechanisms, they may be more inclined to work towards goals, regardless of anxiety-provoking situations on the job [39]. Furthermore, unless coping mechanisms are utilized to help individuals cope with anxiety provoking events, there is a higher chance that these stressors will negatively impact their work performance [120]. Therefore, there is a critical need to target coping mechanisms and coping skills for youth and young adults with ASD because it can promote adult functioning, lead to better mental well-being, and can essentially help with successful job retention.

\subsection{Coping in the Workplace}

In this section of my thesis, I first discuss coping in relation to ASD. More specifically, I discuss the literature on coping with ASD in general, then lead to a discussion on the types of coping strategies used by youth and young adults with ASD. Then, I discuss coping in relation to employment with youth and young adults with ASD. This gives us a broad idea of how coping relates to ASD and allows us to understand the different types of coping strategies reported in the literature. By understanding coping strategies for youth and young adults with ASD in general, we can further uncover the types of coping used in employment. Due to the global pandemic of the Coronavirus (COVID-19), I conclude this section by briefly discussing its recent impact on employment. 


\subsubsection{Coping and ASD}

There is a growing body of literature showing that what a person does to manage and cope with challenges can describe how stress can be magnified or reduced, which ultimately affects one's psychological and physical well-being [120,121]. Erica and Ramon discussed the importance of understanding coping during the adolescent phase of youth and young adults with ASD because all young people go through social and developmental changes during that period of life [122]. For youth and young adults with ASD, this period is reported as specifically challenging due to difficulties with social relationships and constricted patterns of interest [123].

Emotion regulation is another term used in the literature that can characterize coping. Emotion regulation involves analyzing emotions, reacting to that emotion and then finding strategies to regulate that reaction [124]. Common strategies include suppression and ignoring emotional responses and appraisal, where individuals reframe their thoughts in regard to the emotional stimulus [124]. In an analysis of emotion regulation in ASD, Mazefsky et al. found that emotional regulation challenges may be related to the symptomology of ASD because of co-occurring psychiatric conditions associated with ASD, which can potentially impact emotion regulation in youth with ASD [125]. Difficulties with emotion regulation are associated with strong or weak reactivity and can be seen as anger outbursts $[126,127]$. Similarly, studies have suggested that individuals with ASD are more reliant on maladaptive emotion regulation strategies which lead to poorer outcomes such as worse social functioning, higher symptoms of anxiety, and more maladaptive behaviour [127-129]. In an investigation into emotion regulation as a mediator between depressive symptoms in adults with ASD, Sáez-Suanes et al. reported that emotional regulation strategies played an important role in the development of depressive symptoms in adults with ASD (e.g., can be included in depression reduction therapy) [130]. Although there are several studies that look at emotion regulation in individuals with ASD, there is limited literature that focuses on emotion regulation in the context of employment for youth and young adults with ASD.

In the coping literature, research on coping predominantly focuses on parents and caregivers of people with ASD [40,41], with few studies that focus on strategies used by people with ASD [131]. In relation to coping strategies used by people with ASD, Pouw et 
al.'s study reported fewer symptoms of depression when a sample of young boys with ASD used appropriate coping strategies such as seeking social support or finding a solution to an issue at hand [132].

Another study that explored the general experiences with ASD among young women (ages 12-18 years) found coping as a way to mask ASD and to fit in with the neurotypical world [133]. Although masking is reported to be used by many women with ASD, some women express challenges with using masking, which range from constant exhaustion to one individual describing a loss of her own sense of identity [134]. For other youth with ASD, coping can be linked to behavioural and higher emotional problems [135]. These studies illustrate that coping can be used to regulate emotions, fit in with neurotypical peers, and relates to behavioural functioning $[132,133,135,136]$. Collectively, these studies outline a critical role for coping in the workplace. However, there seems to be some evidence to indicate that the burden of adaptation is often placed solely on the individual with ASD, rather than external influences (e.g., workplace rules) adapting or modifying expectations of employees with ASD [137].

\subsubsection{Types of Coping Strategies}

To cope with everyday life, youth with ASD express the advantages of listening to music, reading books, and participating in physical exercises to manage and alleviate feelings of stress [11]. In relation to solving issues at hand, several lines of evidence suggest a robust relationship between stress management strategies and well-being outcomes [10,138,139]. In an investigation into coping with stressful circumstances, Robertson and Frydenberg explored coping strategies used by male adolescents with ASD between the ages of 13-17 years to characterize the type of strategy used and to see its effectiveness [10]. Robertson and Frydenberg found that some male adolescents in their sample reported ignoring, avoiding or accepting the problem as a form of coping [10]. For adults with ASD who frequently ignore, avoid, or accept the problem as a form of coping, several studies found this to be concerning as these strategies were not associated with positive wellbeing outcomes [138-140]. By ignoring, avoiding, or accepting the problem as a form of coping, it can cause a risk of reduced well-being and increased distress if overused or inappropriately used [135,140]. 
Thus, these strategies may be used due to the underlying communication difficulties and social difficulties in ASD, which can make complex social situations unmanageable [10].

There are also positive coping strategies that people with ASD utilize such as social support, seeking support from friends, and looking at the positive in stressful situations $[10,141,142]$. For example, in an exploration into the predictors of successful coping in men with ASD and their wives, Renty and Roeyers reported social support being a source of coping in marital adaptation [141]. In reference to youth with ASD, Robertson and Frydenberg found male adolescents (ages 13-17 years) spending more time doing special interests, looking for relaxing diversions, working hard, and focusing on solving the problem as positive coping strategies [10]. Special interests can be classified as interests that may be atypical by nature or by their intensity used by the individual, which often vary from individual to individual [131]. Another qualitative study analyzed the types of coping styles used by thirty adults with AS or ASD and the findings showed eight different styles of coping [131]. The study reported styles of coping including special interests, viewing disability positively, diagnosis (e.g., allows self-discovery), seeking social support from friends, seeking support from animals, normalization (e.g., normalizing stressful situations), intellectualization, and humour [131]. Together these studies provide evidence that coping strategies can vary by individual, can be used in various contexts, and be multi-faceted.

\subsubsection{Coping in Employment}

Coping can also be utilized in the context of employment as youth and young adults with ASD face stressors (e.g., difficulties communicating with coworkers) in the workplace on a constant basis [26]. For instance, one study reported that in a sample of 66 employed adults (ages 22-55) with ASD, direct communication with supervisors was an important strategy to overcome barriers at work because it allowed participants to clarify issues at hand and resolve the situation immediately [26]. Despite communication difficulties being a barrier to employment for many youth and young adults with ASD [2,73], some adults with ASD report that having direct conversation essentially showcases resilience to adversity [26].

Another study reported that in a sample of 92 adults with ASD, social camouflaging was often used in the context of employment [143]. Social camouflaging refers to strategies used to hide behaviours associated with ASD through techniques to seem socially competent and 
looks for ways to prevent others from observing social difficulties [143]. In this specific study, social camouflaging combined masking and compensation techniques by suppressing, concealing, or controlling behaviours related to ASD that may be seen as inappropriate [143]. In relation to the compensation aspect of social camouflaging, it centred around developing strategies to meet social communication gaps (e.g., making sure to maintain appropriate eye contact during a conversation) [143].

Furthermore, Mcknight-Lizotte found that in a study that discussed work-related barriers for youth with ASD, one participant noted the importance of scripting (e.g., memorizing interview prompts beforehand) during the interview process as a form of coping [73]. Likewise, another participant discussed the advantage of job-training and role-playing as it improved social skills and helped with understanding employer's expectations, which aided with coping in employment [73]. In a study that explored occupational demands for employees with ASD, Hayward, McVilly and Stokes discussed the importance of asking employees with ASD about their job role and the environment they experience to understand circumstances in the workplace that can be problematic [29]. Collectively, these studies outline a critical role of coping in employment. What is less clear is the nature of coping and the types of coping employed for varying challenging circumstances in the workplace. This indicates a need to understand the various perceptions and experiences of coping in employment among youth and young adults with ASD. Although studies discuss factors that can lead to successful employment [22], there is still less emphasis on coping mechanisms in the workplace and how coping impacts employees with ASD. Thus, with this limited literature in personal experiences with coping in employment, it is important to further expand this area of research because coping strategies can be utilized to overcome stress and anxiety in the workplace.

\subsubsection{Importance of Coping}

Understanding how one copes with stress is vital because the utilization of certain coping mechanisms can be associated with outcomes related to positive mental wellbeing, depression, and anxiety-related symptoms. In addition, some youth and young adults with ASD may lack coping strategies or skills to manage stress and anxiety-ridden situations $[10,144]$, especially in a competitive work environment. As individuals with ASD are 
working independently (e.g., no on the job support) in competitive work environments, stressinducing situations (e.g., communication difficulties with managers) can occur on a daily basis, which may magnify feelings of stress and anxiety on the job [29]. One study reported that when adults with ASD experience stress or conflict at work, they tend to quit or skip work without giving early notice to their employers [145]. Thus, it is important to uncover potential coping mechanisms because these can help youth and young adults with ASD relieve stress and anxiety and potentially point to reasons for employment retention. However, there has been no detailed investigation of the experiences of coping with employment-related challenges among youth and young adults with ASD. By uncovering coping mechanisms through individual work-related experiences, it is possible to determine the stress triggers in the workplace, which is also underreported in the literature.

\subsubsection{Impact of COVID-19}

The impact of the COVID-19 pandemic shifted rapidly across the world, infecting millions along the way [146]. Some countries fared better than others in the combat to stifle the virus, but globally, the effect that the virus has on human life and life expectancy is inexplicable [147]. One study reported that for adults with ASD, the COVID-19 pandemic affected important areas of life including employment [148]. Specifically, the impact of the COVID-19 pandemic on employment was lower for adults with ASD who worked full-time in comparison to those who worked part-time or were self-employed [148]. Around the world, many individuals are faced with disruptions in routine, however, adapting to changes may be particularly challenging for adults with ASD during the COVID-19 pandemic [149]. Especially during this period of uncertainty, adults with ASD may be at higher risk for distress [148]. In addition to the impact on employment for adults with ASD, coping in response to the pandemic worsen over a period of time due to disruptions to personal aspects of life (i.e., social and home life) [148]. Therefore, the pandemic can be a reason as to why experiences with finding and sustaining employment are limited now more than ever.

\subsubsection{Potential Implications and Conclusion}

If youth and young adults with ASD develop coping mechanisms, they are more likely to work toward employment goals [39]. This study can help provide coping strategies for people with ASD, which can be used to reduce stress and anxiety in employment. By being informed 
on these different types of coping strategies, they can serve as instruments to assist employees with ASD to better cope with stress and anxiety-related circumstances in employment [120]. Furthermore, this study also helps provide employers with the knowledge of differences in ASD and how to make necessary accommodations in the workplace. For instance, if employers know that individuals become stressed and/or anxious with changes in routine, they can inform employees in advance of schedule changes so that the change is not abrupt [120]. Thus, this demonstrates the importance of uncovering coping mechanisms as employers can better facilitate the mode of coping and help employees with ASD relieve stress and anxiety at their place of employment. In addition, due to the fact that individuals with ASD experience challenges with social interactions, they may have fewer resources to cope with interpersonal relations in an employment setting, which leads to stress [29]. Unless practices are in place to help cope with anxiety-provoking circumstances, it is highly likely that these situations will negatively impact work and performance [120].

\section{In conclusion, the objectives of this study are:}

1. To understand the subjective feelings of stress and anxiety that youth and young adults with ASD experience within competitive employment.

2. To understand subjective coping strategies used by youth and young adults with ASD when faced with work-related stress or anxiety. 


\section{Chapter 3}

\section{$3 \quad$ Methods}

\subsection{Design:}

This study followed an interpretivist paradigm [150] using interpretative phenomenological analysis [151] as a research methodology to conduct qualitative interviews and gain insight on how youth and young adults with ASD cope with stress and anxiety in competitive employment.

\subsection{Research Paradigm: Interpretivism}

Paradigms are beliefs and practices that can help control inquiry in various disciplines [152]. Paradigms can be classified by the ontological, epistemological, and methodological variations which ultimately guide the conduction of research [152]. The reason for choosing an interpretivist paradigm was due to the nature of the research question of this study. Since the goal of this study was to explore and understand the experiences of coping in relation to competitive employment among youth and young adults with ASD, interpretivism was deemed a suitable approach to capture the subjective realities of participants. The interpretivist paradigm assumes reality to be subjective, rather than objective, as individuals are sharing their experiences [153]. Thus, there is no fixed truth or objective reality when the experiences of each participant are discussed; instead, there are multiple realities [153]. The ontology of the interpretivist paradigm focuses on the concept of relativism, which emphasizes that multiple realities of a particular phenomenon are constructed experientially and socially [154]. Furthermore, with this research study, I answered the epistemological question of "how do I know what I know" by having the realities of each individual in my sample observed and understood in a subjective manner. That is, there is essentially no ultimate way of knowing the phenomenon; rather, the subjective realities of my participants showed that there are diverse interpretations of reality [155]. 


\subsection{Theoretical Framework}

Following an interpretivist paradigm, the Transactional Model of Stress and Coping (TMSC) [9] is an essential framework for understanding the barriers and stressful events (e.g., work-related challenges) that youth and young adults with ASD face and how individuals cope with these challenges. In this model, stress is neither solely based in the environment or in the individual but instead, a product of their relationship [9]. The process of coping depends on two encounters. First, the extent to which a person has the capability to manage a stressful environment or situation is influenced by its context, and in this, coping changes from one encounter to another [9]. Second, this psychological stress and coping alters as the encounter unfolds [9]. This emphasizes the fact that coping is very context and situation based, which can impact the coping practice that is utilized. Coping mechanisms can be characterized as emotional (individual tries to alter their emotions), behavioural (individual tries to alter the issue), or cognitive (individual tries to alter their beliefs) [9]. Hence, if a certain situation is deemed difficult by an individual now or at a later time, the individual evaluates their available coping strategies and/or resources and utilizes them situationally $[9,11]$. Coping resources can be characterized by positive beliefs, social support, problemsolving ability, physical health, or material resources $[9,11]$. Thus, reflecting an interpretivist paradigm, reality is based from an individuals' subjective experiences of the world [156]. Furthermore, TMSC falls within the paradigm of interpretivism because it focuses on the individual and how they cope based on their available coping strategy, the context, and the situation in which they encounter stress.

Folkman and Lazarus characterize coping as problem-focused coping or emotion-focused coping [9]. Problem-focused coping essentially attempts to manage or alter the issue that is causing an individual to experience stress [9], hence problem-focused coping strategies are typically used in daily problem-solving. For problem-solving coping strategies, they usually involve figuring out the problem, considering potential solutions, weighing pros and cons of the situation and then selecting a way to approach the situation [9]. Emotion-focused coping can be categorized as ways to reduce or change the negative emotions that become associated with stress [9]. With emotion-focused coping, efforts are made to avoid or minimize a stress and/or anxiety inducing situation [9]. 
This theory was chosen in particular because it focuses on an individualistic perspective on coping as coping is unique and context and situation dependent. It highlights the effects of various stressors which can result in many different types of coping responses. This theory posits that the style and nature of coping is determined by the individual and their established coping strategies for a particular stressful encounter [9]. Furthermore, an advantage of this model is that it gives the individual the control on how much power they have to change a response [157]. Put differently, it gives individuals a chance to evaluate a situation and respond in a way that is beneficial and helpful to them. People are not limited to a core set of strategies because stress is ever changing and individually experienced, thus individuals can appraise a situation to their liking and own capability. Moreover, since the focus of my research is to understand the coping strategies of youth and young adults with ASD, this framework will help conceptualize the influence of barriers to employment [11]. This theory fits within the context of employment and ASD because it demonstrates how coping in an employment setting depends on the context of stress and what strategies are available at the individual's disposal. Furthermore, this framework has been used to analyze parental coping skills on stress in parents of children with ASD $[158,159]$. In relation to youth and young adults, this framework is also used in a study to analyze coping in youth with ASD transitioning to adulthood [11].

This theory was used to frame the research and analysis. In theory framed research, the theoretical framework clarifies what questions will be addressed and how the data will be collected [160]. Similarly, this theory provided a guide for the study and a lens in interpreting the data so that we could analyze information in a significant yet meaningful way [160]. The Transactional Model of Stress and Coping was used in our interview guide to frame questions related to stress and coping. Specifically, questions related to emotion and problem-based coping were prompted during interviews with participants. Furthermore, this framework helped conceptualize the different types of coping, which were important in the interview process as participants could reflect and compartmentalize the types of coping styles employed. In the same manner, this framework was used in the analysis of the data as themes related to stress, anxiety and coping were examined. 


\subsection{Research Methodology: Interpretative Phenomenological Analysis}

Following an interpretivist paradigm, an interpretative phenomenological analysis (IPA) was used to explore and understand how participants coped with stress and anxiety in a competitive employment setting. IPA was first proposed by Jonathan Smith in a paper that argued for an experiential perspective in the field of psychology [161]. The IPA approach is rooted in phenomenology; that is to say, it involves extensive examination of the participant's life in order to explore and understand personal experiences [151]. However, IPA goes beyond phenomenology because it employs a double hermeneutic approach, which includes a process of discovery and interpretation while mainly staying focused on the participant and their experiences $[161,162]$. This methodology aligns with the paradigm of interpretivism because this study explored the subjective realities of participants, which allowed for individual examination of qualitative experiences; thus, no single reality was objectively assessed. This approach fits within the context of employment and ASD because the objective of this study was to ultimately understand individual experiences with work-related stress, anxiety and coping. Hence, IPA aims to understand how participants are making sense of their personal and social world [151]. This approach highlights the role of the researcher as one who tries to get into the participant's world through an interpretive process with the data [151].

IPA was chosen as the research methodology for various reasons. First, this approach focuses on the lived experiences of the participants with the researcher aiming to make sense of each participant's world through a process of reflective inquiry [151]. Second, this approach was utilized because of the important role the researcher plays in the data collection and analysis process [151]. I acknowledged that the participants were experts in their own subjective truths; however, through an IPA approach, I also recognized my role in the data analysis process. In addition, this approach was chosen due to its methodological suitability, specifically the acceptance of the researcher's influence on the data collection and analysis process [151]. The role of the researcher was an essential element of analysis, as I attempted to obtain an insider's perspective of my participants, which eventually led me to be merged into the research process. To ensure that the analysis process was rooted in data, I was 
reflexive and accepted the fact that my thoughts and beliefs were essential to make sense of the personal world of my participants through a process of interpretive activity. Finally, this approach was chosen specifically because it offered direction and guidance on how to approach the phenomena of interest (i.e., how youth and young adults cope with stress and anxiety in competitive employment) for sampling, data collection and analysis [151].

\subsection{Sampling and Recruitment:}

\subsubsection{Sampling Strategy:}

IPA studies are often conducted on smaller samples because the goal of IPA is to understand the detailed experiences of participants as opposed to making general claims about a phenomenon in question [163]. Similarly, since IPA is used to understand a phenomenon through personal experiences, participants are often recruited through purposive sampling to find a distinct group for whom the research question will be important [163]. The power of purposive sampling essentially lies in the selection of information-rich cases to study a phenomenon in an in-depth manner [164]. Utilizing information-rich cases allows researchers to learn extensive detail about the issues of fundamental importance to the question of interest [164]. Typically, we can learn a lot from information-rich cases because we gain insight into the personal experiences of participants, including challenges with maintaining employment for youth and young adults with ASD, which through these interviews, touched on topics such as stress, anxiety, and coping. Since I selected a particular group of individuals (e.g., youth and young adults with ASD), I uncovered this phenomenon of coping in employment through the various lived experiences of my participants and this was possible because I was able to conduct an in-depth study with a small sample size. The sampling strategy remained consistent throughout the study, was not refined based on participation, and saturation was used to help inform this approach. Data saturation can be characterized by a point where no new information can be obtained during the interview process [165]. Specifically, code saturation was used for this study and this approach can be referred to as a point where no new codes are identified in the data set [166]. Essentially, this type of saturation is discussed in relation to codes developed both inductively and through a priori codes [166]. Even though participants had unique and individualized experiences pertaining to stress, anxiety and coping in the workplace due to their differing roles and environments, these experiences often 
fell under the identified subthemes in code saturation. As such, this study reached code saturation after nine interviews, which was evident as interviews ten, eleven and twelve replicated themes identified in earlier interviews and no new themes were found through the remainder of the analysis.

\subsubsection{Recruitment:}

Participants were recruited using the following methods: posting flyers on recruitment websites and on social media, from the following locations: Holland Bloorview Kids Rehabilitation Hospital, ASD-related agencies (e.g., Kerry's Place, Autism Ontario, Community Living Ontario, etc.), and social media platforms (e.g., Facebook, Instagram, Twitter, and LinkedIn). A flyer was distributed electronically and posted at these recruitment sites and those who responded to the recruitment information were emailed and asked distinct questions to assess their eligibility for the study. If participants wanted more clarification about the study, emails were exchanged. Once participants answered the screening questions and were deemed eligible to participate in this study, an information package was sent to read over. This information package discussed the study in greater detail and contained information related to consent to participate (see appendix A). Written consent was obtained directly from the youth or young adult during secure email exchanges.

\subsubsection{Inclusion Criteria for Study Sample:}

I aimed to interview 10-15 youth and young adults with ASD (with equitable gender representation (i.e., equal number of males and females); ages 15-30 years). This sample size was chosen because qualitative studies usually reach data saturation between 12-15 participants [167]. Criteria for study inclusion were as follows: (1) having an ASD diagnosis (i.e., self-reported a professional diagnosis), (2) ages 15-30 years, (3) employed or have competitive work experience, (4) able to read and speak in English, and (5) able to participate in individual interviews (e.g., without additional support). These inclusion criteria were selected for a number of reasons.

First, the purpose of this study was to focus on the personal experiences of youth and young adults with ASD in relation to coping with stress and anxiety in the workplace. Studies in the past have focused on stress and anxiety from the perspectives of caregivers and parents 
of individuals with ASD without highlighting the perspectives of youth and young adults with ASD [40,41], especially in an employment setting. Hence, this study addressed this gap in the literature by focusing on youth and young adults with ASD and understanding their personal experiences with stress and anxiety in the workplace. Second, I selected this age range because it encompasses the transition age from adolescence to early adulthood [13]. Third, I focused on individuals who were currently working or had work experience. The reason for this was because I wanted to capture the experiences of youth and young adults who may have had some type of work experience with varying lengths of employment (e.g., summer employment or recently laid off). In addition, whilst some research has been carried out on employment experiences including studies that focus on pre-employment programs and transition to work, there remains a paucity of evidence on the individual employment experiences, especially among youth and young adults with ASD [33,168]. Thus, this research can help to address the experiences of youth and young adults with stress, anxiety, and coping in competitive employment. Understanding the link between stress, anxiety and coping in employment will help provide new insight on employment experiences among youth and young adults with ASD. Likewise, it was important to address stress and anxiety-related experiences in competitive employment specifically because unlike other forms of employment such as supported employment, employees in competitive employment have no on the job support [7]. On the job support can be characterized by one-on-one support by job coaches or employment specialists [169]. Therefore, it was important to understand the personal and individual experiences with stress, anxiety, and coping for youth and young adults with ASD in competitive employment because they are facing challenges independently in the workplace. Fourth, I focused on English speaking participants because I was the only research member that conducted the interviews for this study, and I along with a few members of my research team (i.e., supervisor and research assistant) analyzed the collected data. Therefore, the project budget did not allow for interpreters. Finally, I wanted participants to be able to partake in interviews independently without additional supports (e.g., parent or caregiver). The reason for this was because I wanted to focus on the direct perspectives and experiences of youth and young adults with ASD with no outside influence from other individuals. 


\subsection{Online Data Collection Procedures}

Data for this study were collected from May 2020 to February 2021. Initially, data were meant to be collected primarily in-person (e.g., in-person interviews) or over the phone. However, due to COVID-19, the in-person data collection process was altered (e.g., primarily using Zoom communications) to reduce the spread of the virus and maintain social distancing. As a result, I was permitted by our ethics board to conduct interviews over Zoom communications, which still allowed face-to-face interactions through video conferencing.

Therefore, I conducted interviews remotely (e.g., over the phone or over Zoom). In total, 11 interviews took place over Zoom and 1 interview took place over the phone (participant preference). For the interview that took place over the phone, I used a recorder to record the audio. I conducted all the interviews for this study and for six of those interviews, a research staff was present to help record the interviews (i.e., over Zoom) and store them securely on the Holland Bloorview Kids Rehabilitation Hospital's secured drive. Of the eleven interviews that took place over Zoom, nine participants turned their camera on for the interview and two participants did not. Specific considerations were made when conducting remote interviews such as securing data to ensure participant confidentiality and anonymity [170]. Once interviews were recorded, they were stored immediately on the secured drive and deleted from the local hard drive to ensure security and participant confidentiality.

\subsubsection{Utilizing Zoom to Conduct Interviews:}

There are advantages to conducting interviews remotely in an online format such as Zoom, including greater disclosure often due to perceived anonymity [171-173]. In addition, by having the option to participate in an interview over the phone or over Zoom, it allowed participants to choose a method with which they felt more comfortable. For example, if an individual chose to participate in an interview over the phone instead of Zoom, it eliminated the chance of internet lag, which in itself may have impaired the interview process. On the other hand, for those that used Zoom, participants may have felt more comfortable with an online visual interaction, especially if sensitive topics were discussed [170]. Zoom allows rapport between researchers and participants, especially when compared to nonvisual media such as telephone or email [174]. Therefore, by having a visual option such as Zoom, 
participants can see the researcher and respond to nonverbal cues (i.e., head nods), which facilitates engagement, builds trust, and promotes a natural, comfortable conversation [174]. Since Zoom provides a video option (i.e., face-to-face), it can provide a more intimate setting than on the phone where there is no face-to-face interaction.

\subsubsection{Interviews:}

Participants took part in individual semi-structured interviews where they were asked open-ended questions related to stress, anxiety, and coping in competitive employment. An initial interview guide adapted from a larger project, of which this study is a part [175], was utilized for this study. The purpose of the broader study was to explore how and when young people with disabilities disclosed their condition and requested workplace accommodations [175]. Since this larger project did not specifically focus on youth and young adults with ASD, the interview guide was modified to make it more specific and relatable to this particular study. The interview guide (see appendix B) was modified after a vast literature search on employment for youth and young adults with ASD, coping for youth and young adults with ASD, and stress and anxiety in youth and young adults with ASD.

The design of the semi-structured interviews was also influenced by the IPA approach to methodology. IPA researchers often wish to collect and analyze data in detail to better understand how participants perceive and make sense of their subjective realities [151]. As such, IPA studies typically rely on semi-structured interviews as a method of data collection. With semi-structured interviews, it allows the researcher and participant to engage in a conversational dialogue whereby initial questions are altered in light of participant's responses and the researcher is given room and flexibility to probe areas of interest which arise during the conversation [151]. Overall, the questions on the initial interview guide remained the same, however, I added question prompts to make the interview guide more specific to this study. Question prompts included questions related to challenges faced in the workplace, examples of coping in the workplace, coworker and supervisor relationships, overcoming barriers in the workplace, and challenges with asking for accommodations at work. Since this interview guide was formatted in a way to allow open-ended conversation, I added probing questions to further investigate and capture work-related experiences for youth and young adults with ASD. Probes were utilized to encourage discussion related to coping 
strategies employed in the workplace, experiences with stress and anxiety in the workplace, and personal experiences with accommodations and disability disclosure in a workplace setting. The TMSC was used to frame question prompts related to stress and coping in the workplace. In particular, question prompts characterizing problem-focused coping and emotion-focused coping were added to the interview guide.

Before interviews were conducted with participants for this study, the interview guide was pilot tested with a youth mentor (i.e., a person who speaks about their own life experience and answers questions related to living with a disability [176]) with ASD. This pilot-testing was done to ensure comprehensiveness and clarity of questions in the interview guide. Pilottesting the interview with the youth mentor allowed me to: (1) gain perspective on whether the questions were clear and understandable, (2) decide the relevancy of each question, (3) determine whether question probes added depth to the conversation and information gathered, and finally (4) to gage how youth and young adults would react to ASD and employment related questions (e.g., asking for accommodations). After pilot-testing, I modified the questions accordingly (e.g., rewording the questions to ensure clarity), however, no questions were added or removed before applying the interview guide to participants.

First, the interviews opened with demographic questions related to gender and type of disability. The interviews then extended to questions related to employment status, challenges in finding employment, disability disclosure and accommodations, gender in the workplace, and advice for those who are having trouble finding employment. These questions were designed to obtain information related to participants' work experiences, stress management at work, and potential coping mechanisms utilized to manage stressful situations in the workplace. By engaging in conversation with the research participants, I was able to explore the experiences of stress and anxiety in the workplace and how youth and young adults with ASD cope with the demanding work life. Interviews were conducted in an interactive, conversational style to help set a comfortable setting for the research participant. Research participants are deemed the experts on their own personal experiences regarding stress and anxiety in competitive employment. Therefore, interviews were conducted in a manner that allowed research participants to recount their experiences in their own way [177]. 
The one-on-one interviews ranged from 45 to 104 minutes in length (mean 66 minutes). All interviews were audio recorded and later transcribed verbatim, which allowed for the capturing of participants' nuances (e.g., pauses and non-verbal cues) [177]. For IPA studies, the level of transcription is usually at the semantic level; that is, transcripts include all spoken words including false starts, significant pauses, and laughs [163].

In addition, I kept a field note journal during the interview process [73,178] (see appendix $\mathrm{C}$ for example journal template). Field notes were utilized during interviews because if too much time was devoted to writing notes, the deeper meanings and experiences of my research participants may have been missed [178]. Similarly, if I attempted to write everything down, it would potentially interfere with establishing rapport with the participant [163]. Therefore, field notes comprised of concise jotted notes, phrases, and non-verbal cues, which served as reminders of key dialogue in every interview [178]. Every participant received a $\$ 10$ honorarium to recognize their contribution to my research study, as expected by the research ethics committee.

\subsection{Ethical Considerations:}

Ethics approval was obtained from the Research Ethics Boards at Holland Bloorview Kids Rehabilitation Hospital and the University of Toronto (see appendix D). In addition, I recognized that in relation to procedural ethics (formal approval needed for a study to commence [179]), I also considered the principles of beneficence (analyze risks and benefits), justice (equity), and respect for persons (privacy and confidentiality) throughout the research process [180]. Furthermore, all consent forms, verbal, and written interaction with and to research participants, e-mail records, phone communication, and any form of communication were conducted under these considerations.

Likewise, I ensured the privacy and confidentiality of all participants throughout the research and after the research process was concluded. Prior to my interviews, I asked participants if they consent to their information being used (e.g., quotations) from the transcripts in the context of my study. As a result, I ensured that confidentiality and privacy were retained throughout the research process as pseudonyms were used in place of names of the participants. In regard to written consent forms, I utilized forms with plain language to 
ensure transparency and clarity to my participants. Participants signed the consent forms and sent back an electronic copy. Signed consent forms simply provide evidence, thus as researchers, we explained the premise of our study, what participation would include, and gave participants the ultimate decision on whether they wanted to participate [179].

To elaborate, we recognized the complex dynamics between the researcher and research participants in this study. This dynamic related to situational ethics, which can be characterized by unpredictable, yet ethically essential moments that occurred during the study [181]. These important moments can occur between researcher and research participants which may be difficult to confront [179]. For example, there were situation(s) where I needed to probe a participant about a difficult experience regarding coping in the workplace. These circumstances can potentially lead to individuals feeling overwhelmed and/or cautious to openly discuss personal information. However, in these cases, I made sure that trust and privacy were maintained to allow research participants to openly talk about their experiences. In addition, due to the nature of these conversations, I provided resources for individuals if the need arose (e.g., counselors, psychologists, and debriefing sessions). There may be other instances where participants were unwilling to provide information. For example, if a participant felt uncomfortable discussing certain topics (e.g., death in relation to coping), I moved on from that topic and returned to it later in the interview.

In regard to ethical issues that may occur beyond the study, I ensured that the information given by our research participants was used to enhance the research. We did not take information out of context or mislead/trick our participants with our findings [181]. We remained transparent with our participants and if questions or concerns arose throughout the interview process, participants had the right to contact us and withdraw from the process at any time.

\subsection{Analysis}

\subsubsection{Approach to data analysis:}

I applied an IPA approach to the data analysis process to obtain an in-depth understanding of participant experiences [163]. Specifically, I used IPA to explore how youth 
and young adults with ASD managed stress and anxiety in competitive employment and how they utilized coping mechanisms in response to these stressful situations.

Analysis began with a close examination of the first transcript, which I read multiple times with the left-hand margin employed as a place of annotation (e.g., electronically) to indicate anything significant a respondent said [163]. This first stage of analysis was fundamental because reading and rereading the transcripts multiple times allowed me to become more familiar with the text [163]. In addition, during this stage, I made comments to summarize/paraphrase the participants' response, which indicated the use of language in context with its associated content. The aim of this first step of analysis was to familiarize and immerse myself with the data [163] and by doing so, I managed to create preliminary notes. Each reading and rereading of the text provided new insight for my analysis $[163,182]$. I then moved onto the second stage of analysis which involved the transformation of notes into themes $[163,182]$. To clarify, I recognize that themes are not emerging from the data set, rather I play an integral role in uncovering and constructing the themes through an interpretative and reflexive approach to analysis. A hybrid approach (i.e., utilizing both inductive and deductive coding approaches) was also used in this analysis. A deductive approach can be characterized by a top-down approach to coding by utilizing a priori template codes whereas an inductive approach uses data to derive codes [183]. A hybrid approach was used for this analysis because this approach complemented the question of interest (e.g., how do youth and young adults with ASD cope with stress and anxiety in competitive employment?) by allowing elements of the TMSC to be integral to the process of deductive coding while still allowing for themes to "emerge" directly from the data using inductive coding $[183,184]$. This approach to analysis is often well-suited for exploring emerging patterns when there is not a substantial body of literature regarding a phenomena of interest (i.e., coping in competitive employment) [185]. Furthermore, a hybrid approach for a data set can help with identifying basic elements of the raw data through deductive a priori template codes but also allows for flexibility in uncovering meaning through the data [186]. This analytic process goes outside of identifying initial themes on a semantic level because it involves a process to understand and explore latent meanings [186]. Therefore, to understand 
how youth and young adults with ASD cope with stress and anxiety, a hybrid approach was deemed appropriate.

For this study, the TMSC was used in our interview guide to frame question prompts specifically related to emotion and problem-focused coping. Since this framework helped with conceptualizing the emotion and problem-focused coping in the interviews, themes and subthemes related to coping followed a deductive approach as there were subthemes categorized as emotion- or problem-focused coping. By following a deductive approach in regard to coping mechanisms, it can help with characterizing the types of coping styles used, the context in which they are used, and allows for comparisons between problem and emotion-focused coping. During the interviews, I prompted the concepts of problem- and emotion-focused coping especially when participants talked about their stress management strategies. Hence, since I used this theory in my interview guide, it was expected that coping was going to be oriented as problem- and emotion-focused. However, I was able to safeguard the risk of potential overreaching of the orienting theory in data analysis by allowing participants to reflect on their experiences of coping before prompting problem- and emotion-focused coping.

Similarly, subthemes related to disability disclosure and accommodations also followed deductive coding as these concepts were discussed in the interviews. Once again, by utilizing a deductive approach, these a priori template codes (i.e., disability disclosure and accommodations) contributed to my understanding of participant experiences with each respective process. That is, by using a deductive approach, it allowed for a deeper understanding of the processes of disability disclosure and asking for accommodations and demonstrated how each participant can have varying experiences to each process. Once I created these preliminary themes, I made short/concise phrases grounded in the participant's account from the preliminary notes that I created in the first stage of analysis $[163,182]$. This transformation of initial notes was continued throughout the whole transcript.

The third stage of analysis involved seeking relationships between these themes, which eventually led to clustering these themes together $[163,182]$. Essentially at this stage of analysis, I looked for connections between these themes and grouped them together based on similarities and created their respective descriptive labels. These main themes gave insight 
into answering the research question and helped discuss the similar and unique ways in which youth and young adults with ASD cope with challenges related to stress and anxiety in competitive employment. Therefore, at the end of this stage, I created a final list of themes and subthemes for the initial transcript. This stage of analysis is iterative as it involved a close interaction between the reader and text to ensure that the generated themes reflected and related to the actual words of the participants $[163,182]$. Once these themes and subthemes were manually created, I used a coding software (i.e., NVivo) to tag and name certain portions of the text within the transcripts. This ensured that the actual data extracted from the text were coded correctly, leaving in the context of conversation if necessary. In addition, by using NVivo, it allowed transparency in my coding and provided an audit trail of all the decisions that were made.

Once I created themes and subthemes for the first transcript, I then moved on to the next transcript and repeated the IPA steps from the beginning. Although I had themes and subthemes from the initial transcript, I wanted to acknowledge new issues emerging as I went from transcript to transcript. Therefore, as I moved from transcript to transcript, I made notes of new themes and issues and recognized the similarities and differences in the data. The benefit of approaching the new data from the beginning while being mindful of the preexisting themes and subthemes was that it was easier to identify what was new and different in the following transcripts.

At the fourth stage of analysis, I constructed a table of themes (see appendix E for sample theme table) by continuous pattern-seeking among the first few transcripts $[163,182]$. In this stage of analysis, I looked for themes and qualities that were identifiable across the participants. However, at the same time, I also highlighted and made note of differences between participants. In addition, I identified each theme and explained its narrative by relating it back to how youth and young adults with ASD cope with stress and anxiety in competitive employment. Specifically, I identified representative quotations to depict the themes.

My research team, which included the supervisor and lab research assistant with extensive experience in qualitative research methods also helped with the analysis for this 
thesis. My research team assisted with the first four stages of analysis for over $50 \%$ of the transcripts. Once each coder (i.e., supervisor, research assistant and I) established preliminary themes independently, we then discussed the themes and arrived at consensus to continue at our regular meetings. The themes that the research team agreed upon were used to code the remaining transcripts as it helped orient the subsequent analysis.

In the final stage of analysis, with the additional help and consensus of my research team, I identified the final themes and wrote them up one by one $[163,182]$. I described and exemplified each theme using extracts from the interviews, followed by analytical comments $[163,182]$. The rationale for richly describing each theme was to explain to the reader the important experiential items found in the process of analysis $[163,182]$. Therefore, the final paper included the participant's account of their experience and our interpretive commentary.

\subsection{Reflexivity and Positionality:}

In the IPA approach, the researcher's prior conceptions are not disregarded and omitted, but instead are revealed throughout the research process [187]. In addition, in this research methodology, the researcher is known to be an integral part of the analysis and essentially becomes part of the research process $[163,182]$. As a result, we acknowledged and were mindful of our own perceptions, experiences, and beliefs and practiced reflexivity throughout this process. Reflexivity essentially describes the process of recognizing and acknowledging our biases as researchers to the research process [188]. The role of the researcher was vital in inferring the participant's sense making in IPA. To ensure that interpretations were grounded in participant's discourse, I utilized quotations from the transcriptions. Throughout this research process, we recognized that our experiences and knowledge influenced our analysis of the data.

We played an active role as researchers as we continuously created links between our themes and the experiences recollected from the interviews. Our objective as researchers was to explore and understand the experiences and challenges regarding stress and anxiety that employees with ASD face in competitive employment. Thus, we played an important part to gather and analyze data and we recognized our role in the research process [187]. 
It was for this reason that IPA was exceptionally suitable for this study because as a young female without ASD, I would never experience the same world as my participants and yet my own experiences and beliefs may have affected the interpretation of the data set. The ways in which I see the world and ASD specifically impacted the ways in which I viewed and analyzed this data. I am a 25-year-old Tamil-Canadian female in a graduate program studying rehabilitation sciences. I have always been interested in this population growing up because I have first-hand experience with a brother who has ASD. In a time and era where programs and services were limited for families with ASD, this essentially impacted my view and understanding of rehabilitation services growing up. Throughout my life, I wanted to engage and participate in initiatives that helped and enriched lives of individuals with intellectual disabilities such as ASD. In my undergraduate degree, I volunteered for a program that helped individuals with developmental disabilities participate in social activities at the University of Toronto Mississauga Campus. This program is designed to help provide individuals with intellectual disabilities a university-like experience. Specifically, program participants and university students both participate in a variety of social activities held on campus (e.g., playing basketball at the gym). After volunteering for this program for three years, I realized that I was making a large impact on many participants enrolled in the program. From helping improve verbal skills to building close friendships, this program truly opened my eyes by wanting to further enrich the lives of individuals with intellectual disabilities.

After I graduated from the University of Toronto, I worked as a job coach (i.e., provided on the job support one-to-one support) for individuals with intellectual disabilities. I truly believe that working as a job coach further piqued my interest in this field of research because I had direct experience with obtaining employment opportunities for individuals with intellectual disabilities. Since I was a job coach, individuals that I supported rarely encountered challenges with accommodations in the workplace as employers were usually well aware of intellectual disabilities and inclusive hiring. So, through that experience, I started thinking of how asking for accommodations and disability disclosure would change for an individual working independently with no on the job supports. In addition, this also made me reflect on how vulnerable and intimidating both processes can be in an employment setting, especially when employers are not well-informed on intellectual disabilities. 
In reflecting on my personal and professional experiences with employment and ASD, it has become apparent that my own personal biases and thoughts play a pivotal role in this research study. In being so closely connected with individuals with ASD, both in my personal life and my professional life, it made me more aware and connected to the issues and challenges brought up during these interviews in this study. Being reflexive is extremely vital because it allowed for more personal and contextual details, which is essential in providing voices to participant experiences. Furthermore, I was able to recognize the importance of providing a safe and welcoming space for participants as they often spoke on very emotional and personal experiences with employment.

After reflecting on my role as a researcher and highlighting my personal and professional experiences which could have affected my interpretations of the findings, I understand how important and necessary reflexivity is for data analysis because this data may have been interpreted differently by another researcher with a different background. In addition, I recognize that my own judgements, practices, and beliefs may have influenced the way I interpreted and analyzed the data set. I understand that my experiences may have shaped the way I view the data set, especially since I have first-hand experience working with individuals with intellectual disabilities. Thus, my analysis of the data may have been influenced by my academic, professional and personal experiences. This is precisely why I have chosen to use IPA and include a reflexive piece in this master's dissertation.

\subsection{Trustworthiness and Rigour:}

Strategies such as credibility, transferability, dependability, and confirmability were used in this study to ensure trustworthiness and rigour [189]. Credibility is essential as it seeks to address truth value through lived human experiences [189]. Therefore, the researcher's responsibility is to portray the multiple realities of participants as effectively as possible and be submersed into the data set to identify and verify patterns [189]. For instance, I was the sole interviewer for this study and in the same manner, I transcribed all twelve of the audiotaped interviews. This process allowed me to be fully aware of the data set and understand the data's context and make connections through this immersion. Transferability refers to the extent to which results of qualitative research can be transferred to other settings 
and situations [190]. In other words, transferability seeks to provide rich, descriptive data to determine whether findings can be generalized [189]. In this study, I kept a record of the participants' rich, descriptive to help inform discussions of whether the results can be transferred to other settings and populations. Dependability refers to the stability and consistency of findings over a period of time [190]. For this study, I used peer examination (i.e., having multiple people review transcripts) to help increase dependability [189]. Specifically, I met with my research team to discuss themes and subthemes identified in the data set. By doing so, it allowed for other researchers to discuss and provide input on the themes identified. Finally, confirmability refers to the extent to which the results of the study can be confirmed by other researchers [189]. In the confirmability process, it seeks to address neutrality by an external auditor attempting to follow the research process and to understand how decisions were made [189]. For my study in particular, this was achieved by having a second coder (principal investigator) who read over $50 \%$ of the transcripts and developed their own preliminary themes and subthemes. Afterwards, themes and codes were compared and contrasted among the research team (principal investigator and research assistant) to ensure the finalized themes reflected the participant's experience and that the interpretations of the findings were derived from the data [189]. 


\section{Chapter 4}

\section{Results:}

\subsection{Overview:}

This study included a purposive sample of twelve youth and young adults with ASD.

Specifically, there were seven males and five females with ages ranging from eighteen to 30 years who live in Canada (see Table 1). A detailed analysis of the data (see sample coding in Appendix F) revealed three themes relating to the experiences of youth and young adults with ASD as they cope with stress and anxiety in competitive employment: (1) salient workplace factors; (2) stress and/or anxiety inducers; and (3) coping mechanisms (see Table 2). To conceptualize the themes in a visual format, a thematic map was created (see Figure 1). In addition, it is important to note that data was analyzed for gender differences, however there were no differences between genders in the reported experiences. 
Table 1: Participant Characteristics

\begin{tabular}{|c|c|c|c|c|c|c|c|}
\hline $\begin{array}{c}\text { Participant } \\
\#\end{array}$ & Gender & Diagnosis & $\begin{array}{l}\text { Work Status } \\
\text { (Current) }\end{array}$ & $\begin{array}{c}\text { Work Status } \\
\text { (Previous) }\end{array}$ & Type of Employment & $\begin{array}{l}\text { Disclosed } \\
\text { Disability }\end{array}$ & $\begin{array}{l}\text { Asked for } \\
\text { Workplace } \\
\text { Adjustments }\end{array}$ \\
\hline 1 & Male & $\begin{array}{l}\text { High Functioning } \\
\text { ASD and ADHD }\end{array}$ & Unemployed & $\begin{array}{l}\text { Full-time } \\
\text { (summer) }\end{array}$ & Investment company & No* & Yes \\
\hline 2 & Male & $\begin{array}{l}\text { ASD and General } \\
\text { Anxiety Disorder }\end{array}$ & Laid off & Full-time & Auto body shop & No & No \\
\hline 3 & Male & $\begin{array}{l}\text { ASD and muscular } \\
\text { dystrophy }\end{array}$ & Part-time & - & Software company & Yes & Yes \\
\hline 4 & Male & $\begin{array}{l}\text { Asperger's syndrome } \\
\text { and High Anxiety }\end{array}$ & Part-time & - & Grocery store & Yes & Yes \\
\hline 5 & Female & ASD & Part-time & - & Fast food restaurant & Yes & Yes \\
\hline 6 & Female & ASD & Part-time & - & Research & Yes & No \\
\hline 7 & Male & Asperger's syndrome & Part-time & - & $\begin{array}{l}\text { Non-profit } \\
\text { organization }\end{array}$ & Yes & Yes \\
\hline 8 & Female & $\begin{array}{c}\text { Asperger's syndrome } \\
\text { with cognitive and } \\
\text { language processing } \\
\text { delay }\end{array}$ & Full-time & - & $\begin{array}{l}\text { Non-profit } \\
\text { organization }\end{array}$ & No* & No \\
\hline 9 & Female & $\begin{array}{l}\text { ASD, ADHD, } \\
\text { learning disability in } \\
\text { reading and spelling, } \\
\text { social anxiety, } \\
\text { ulcerative colitis }\end{array}$ & Full-time & - & Teacher & Yes & Yes \\
\hline 10 & Female & ASD & Unemployed & Part-time & Grocery store & Yes & Yes \\
\hline 11 & Male & ASD & Part-time & - & Cleaning business & No** & No \\
\hline 12 & Male & $\begin{array}{l}\text { ASD and mild to } \\
\text { moderate hearing loss }\end{array}$ & Unemployed & Part-time & Retail store & Yes & Yes \\
\hline
\end{tabular}

\footnotetext{
*= participants did not explicitly disclose; however, employer may had been informed by employment program that matched participant to work role
}

$* *=$ participant's father disclosed disability to participant's employer 
Figure 1: Relationships Between the Identified Themes and Subthemes

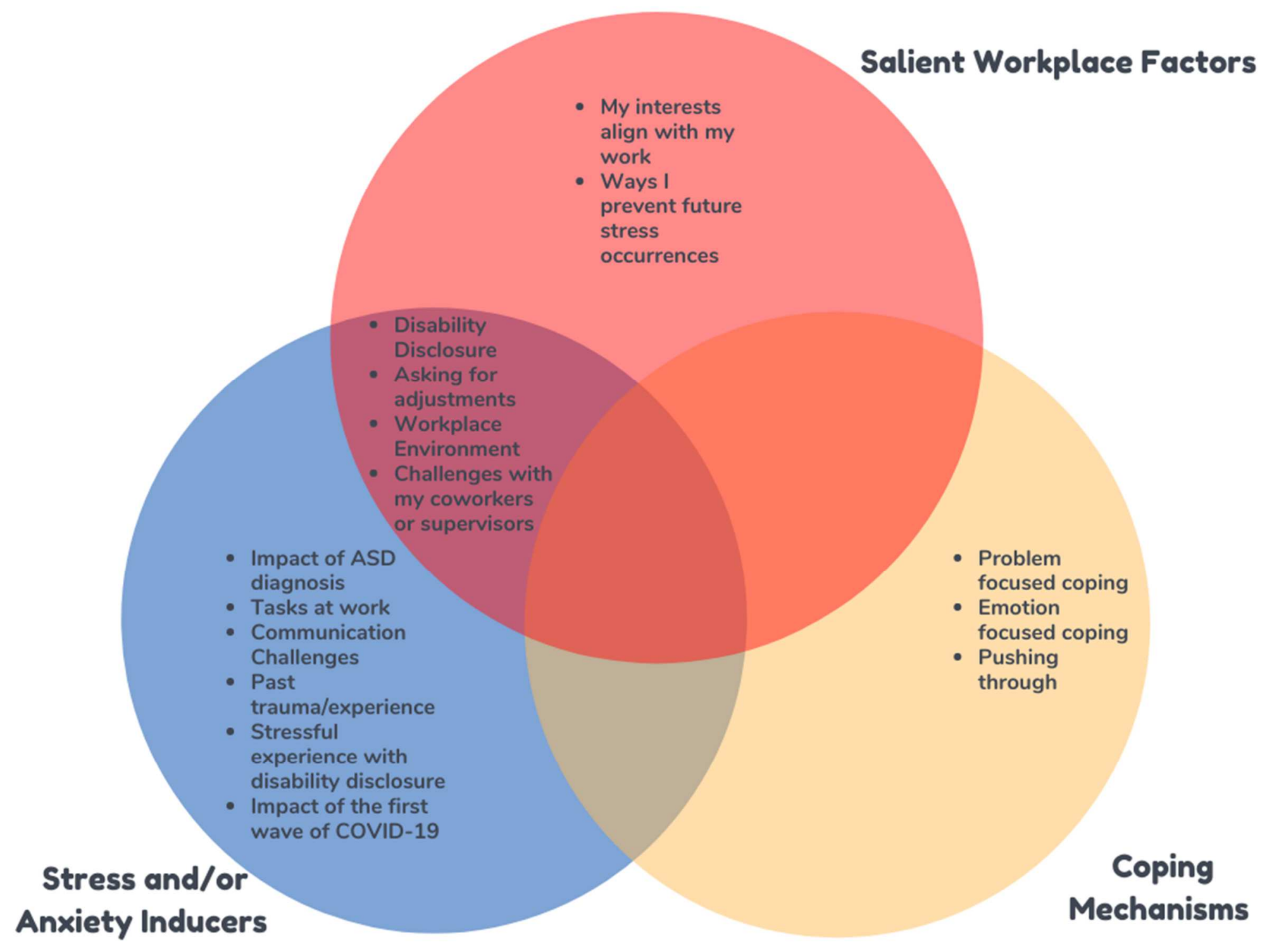


Table 2: Overview of Themes and Subthemes

\begin{tabular}{|c|c|c|}
\hline Theme 1: Salient Workplace Factors & Theme 2: Stress and/or anxiety inducers & Theme 3: Coping Mechanisms \\
\hline
\end{tabular}




\subsection{Theme 1: Salient Workplace Factors}

Salient workplace factors can be characterized by various aspects in the workplace environment that can contribute to the overall positive or negative experiences that participants have on the job. This theme is comprised of six subthemes, each illustrating various participants' experiences with positive and negative aspects in a workplace setting. The theme of salient workplace factors begins with the subtheme of "disclosing a disability in the workplace", where participants described why disclosing a disability can be difficult and overwhelming. In addition, in this subsection, several participants recounted their positive experiences with disclosing a disability on the job. Following disability disclosure, participants usually discussed their needs and adjustments in the workplace to their employer. Hence, the next subtheme is "my experience with asking for workplace adjustments". In this subtheme, participants described their experiences with asking for workplace requests/modifications from their employer. The third subtheme is titled "my workplace environment" where participants gave reasons why they did or did not enjoy working at their respective place of employment. The next subtheme is titled "challenges with my coworkers or supervisor", where participants described their negative experiences with interacting with their coworkers or supervisors on the job. The fifth subtheme is titled "my interests and preferences align with my work", where participants commented on their interests and preferences and how those interests and preferences aligned with their employment role. The final subtheme of salient workplace factors is called "ways I prevent future stress occurrences" and in this section, participants described their personal methods of preventing stress at their respective place of employment. Collectively, these subthemes help to conceptualize and convey the types of positive and negative elements in the workplace environment. In addition, these subthemes help exemplify the personal experiences and workplace environments that participants learn to cope with and adapt to. Below, each subtheme is discussed in further detail, using supportive, representative quotations from participants. 


\subsubsection{Disclosing a Disability in the Workplace}

\subsubsection{Potential Risks to Disability Disclosure}

When the topic of disability disclosure came up in the interviews, nine participants described the potential risks to disclosing a disability in the workplace. Commenting on disability disclosure, one of the participants mentioned that disability disclosure can be daunting due to being unable to manage how the other person responds. For example, "the inability to control how the other person responds... Uhhh ya, I guess brave enough and being self-assured, self-confident enough to actively disclose it, then be prepared to admit that...the struggle" (\#1, male). This emphasizes the caution around disability disclosure because participants may also be weary of discussing the process of disclosing a disability. When asked if the process of disability disclosure can constitute to feelings of stress and/or anxiety, the participant responded with: "For sure yes, definitely. It's definitely a source of... selfconscious insecurity" (\#1, male).

Similarly, for some participants, the process of disability disclosure can be intimidating due to the fear of being punished by superiors in the workplace. As one participant put it:

"I think the best way is ...just to have that feeling that...you can bring something up to your boss without fear of retribution like say hey...we should ...you should be able to solve this in a different way or you should talk to this coworker about such and such you know or whatever you know without fear of being fired or punished for saying...for speaking out.” (\#2, male)

This participant highlighted the fear that may follow with disability disclosure, especially when speaking out and being vocal about issues in the workplace. Participants may be scared to bring forward complaints to supervisors due to fear of retribution from other coworkers.

In a similar manner, participants were fearful of discrimination in the workplace due to disclosing their disability. Talking about this issue, one participant explained that she preferred not to disclose her disability frequently as a result of people being ableist in society:

"I don't disclose it a lot because people are ableist as hell and every response I get is basically like "no you're not, you're nothing like my sister's friend's four year old child" and 
I'm like "is that because they are four? Is it because they are four and I'm 30..." (\#6 female)

Soon after, this participant further commented on the difficulty with disclosing because of common misconceptions that individuals in her environment had about ASD and how the disclosure process is also heavily dependent on the employer:

"If you can disclose that you have a disability, you're too smart to have a disability. Umm, which is very frustrating, there are so many risks to disclosure because... disclosure being safe and being beneficial is dependent on your boss not being ableist and in an ableist society..." (\#6, female)

The comment above illustrates the important role employers play in the disability disclosure process. Participants mentioned how the behaviours and attitudes of employers can impact the process of disability disclosure as some participants described risks to disclosure including misconceptions and biases of employers that can affect one's employability. One participant shared this sentiment when he described his thoughts on disability disclosure and how employers would view him as a job applicant if he disclosed his disability: "they would probably see me differently...they would treat me differently” (\#11, male).

Overall, several participants commented on the risks of disability disclosure in the workplace and how these risks can affect one's employability, their experiences in the workplace, and how these risks can eventually lead to stress and/or anxiety on the job.

\subsubsection{My Positive Experience with Disclosing a Disability in the Workplace}

Although there may be potential risks with disclosing a disability on the job, several participants in this study spoke on their positive experience with disclosing a disability at work. Out of ten participants that disclosed their disability to their employer, five participants recalled that they had a positive experience with this process. For those that had a positive experience, they described feeling relieved once they disclosed their disability to their employer. Another participant expressed feeling more confident once he disclosed his disability to his employer. 
Some participants explained that it was important to have a positive relationship with their employer before they disclosed their disability. One participant echoed this sentiment when she described feeling confident and comfortable enough to disclose her disability once she built a trusting relationship with her boss:

"Umm, I felt like I can trust her and I wanted to complain about ODSP [laughs] and explain to her why my painted structure week to week can be a little weird...that was lovely! She was like fucking...like it is such a relief...the fact that I have a trustworthy boss who knows that I'm autistic means like... I can now tell her how autism affects my performance without having her judge me for it." (\#6, female)

In this case, the participant needed to feel secure and wanted to ensure that she could trust her boss with such vulnerable information. Once that trust was built, this participant disclosed her disability, which made her feel more at ease and happy about her positive relationship with her supervisor.

For some participants, they revealed that they felt more comfortable to disclose once they knew that their employers hired inclusively. One participant described this very notion as he recalled his experience with disability disclosure:

"Ya, so the people I work with like most of them have disabilities...Ya like I said before, like a large amount of people working there like know the different challenges we have so...so I wasn't too worried about... about sharing that...so it felt good that I wouldn't...that I wouldn't have to worry about it [interviewer agrees] ... that I wouldn't have to worry about it later on." (\#3, male)

This participant emphasized the importance of having employers be aware of disabilities and explained ways to make an environment inclusive. In this particular situation, this participant was not worried to disclose his disability as it was a common practice at his place of employment, thus there was trust and a sense of comfortability between this participant and his employer.

Overall, several participants described their disability disclosure process as positive and a relieving experience. These participants described how important it was to have a trusting 
relationship with their respective employer to better facilitate the disability disclosure process. In summary, the results from this study show how the environment and people in the workplace (e.g., supervisors and customers) play a vital role in the varying experiences of disclosing a disability in the workplace.

\subsubsection{My Experience with Asking for Workplace Adjustments}

\subsubsection{My Negative Experience with Asking for Workplace Adjustments}

For youth and young adults with ASD in competitive employment, asking for workplace adjustments can be a challenging process. Out of ten participants that asked for workplace adjustments, five reported having a negative experience (one of whom also had a positive experience with asking for adjustments on the job). Workplace adjustments included the following: requesting time off, scheduling different shifts, taking extra breaks, and trying out different roles within the workplace. Some participants described being scheduled for work irregularly despite asking for a consistent work schedule. One participant recounted their experience with getting scheduled for work despite saying they were unavailable to work:

"but I did find that it was really difficult to get accommodations for scheduling... but the odd thing I noticed was I get scheduled for Tuesday on a regular basis anyways and I had to keep going...I said I can't work on Tuesdays...it's in the system." (\#12, male)

Similarly, another participant commented on their experience with being denied for requesting more days off of work:

"I asked for it frequently throughout my... my work process, just saying you know if I can take the day off...I guess it would be more days off and uhh...it wasn't super scheduled, but I guess it was asking for [inaudible] [interviewer says okay] and uhh there was some push back on that initially..."(\#7, male)

This participant also narrated their negative experience when asking for a work-from-home option from their supervisor in a non-profit sector during the peak of the first wave of the coronavirus (COVID-19) pandemic (March-June 2020). As this participant put it: "umm, well with COVID, I thought it was a shoo-in to say you know not everyone needs to be at the office... but I'm pretty sure that they had a mentality of butts in seats" (\#7, male). Even though 
this participant wanted to work from home during this unprecedented time, his employer refused his requests.

Another participant mentioned that asking for workplace adjustments was not possible for his particular job role because adjustments on the job would essentially equate to a completely different work role. To illustrate:

"With my experience so far, finding accommodations for experiencing tons of workload have not been feasible... there was no way I could ask for my duties to be altered because it would mean asking for a new position altogether, and the company made it clear that they did not see me working in any other position." (\#2, male)

These results indicate that several participants who asked for adjustments on the job described negative experiences with asking for workplace adjustments from their employers. This subtheme suggests that if adjustments are refused or are not taken seriously by employers, it can eventually lead to stress on the job.

\subsubsection{My Positive Experience with Asking for Workplace Adjustments}

Despite several accounts of negative experiences with asking for workplace adjustments on the job, five participants reported that their personal experiences with asking for adjustments in the workplace contributed to their positive attitude towards employment and overall productivity at work. Some participants asked for more flexibility with their tasks (e.g., tentative completion date for tasks) while other participants asked for flexibility in their work schedule (e.g., work for a set number of hours during the week, which can be allocated according to participant's preference). One participant described his experience with asking for accommodations by drawing up a summary sheet of how he worked best in an employment setting:

"Umm for the very first year I worked there, the very first summer, I worked with [program coordinator] to make some sort of handout that summarizes like what helps me do my best work so like physical stuff...umm instruction-based learning... all that stuff ...how I learn best and all that. So, the things I did were asked to use a stand-up desk which I later found that they have that there for all employees. It's just a normal thing there, there's that. Umm I asked for more clear instructions because sometimes I have 
trouble telling...I have trouble understanding ambiguous instructions...things like that so as a summary overall, basically I made a sheet that outlines basic learning strategies and skills that I use." (\#1, male)

Having a summary sheet gave this participant an opportunity to address modifications needed from his employer in order to succeed at work. With this summary sheet, it allowed for this participant's employer to understand what adjustments the participant needed and the associated significance of each adjustment.

Another participant who described having a negative experience with asking for workplace adjustments from his employer (e.g., adjusting schedules for work) also reported having a positive experience with asking for workplace adjustments. Specifically, he described having a positive experience with asking for adjustments on the job when he told his employer that he would not be able to wear handheld transceivers due to wearing hearing aids:

"They have these walkies (handheld transceivers) the staff used to talk to each other. I can't wear those because I have hearing aids, there wasn't any way for me to get the headset on the same time...that was actually part of the reason why I was put on the register.” (\#12, male)

This participant was unable to wear handheld transceivers at work due to his hearing aids and as a result, his employer accommodated his requests by placing him in another role in the store where he did not require handheld receivers.

Adjustments also extended to asking for leaves of absence from work. For example, one participant described their experience with asking for a leave of absence during the peaks of COVID-19 when certain rules and regulations were not enforced:

"Well, I actually took a leave of absence and I'm just returning back to work in the next week. [interviewer says okay] I kind of have some bad history with physical health so I was worried and I had an incident in the last year in which I went blind in one eye temporarily [interviewer says okay] and as it turns out, my immune system was attacking my body [interviewer says oh no]. So, to try to lower the risk until mandates...certain 
mandates were passed, umm I talked to my work and requested a leave of absence and they were like, “cool go ahead.", (\#4, male)

This participant's employer understood why this participant needed a leave of absence and was very accommodating to his request. Together these results indicate that nearly half of the participants described their experiences with asking for workplace adjustments and accommodations from their employer as positive and very helpful, which contributed to their positive experiences at work.

\subsubsection{My Workplace Environment}

\subsubsection{My Negative Workplace Environment}

Four out of twelve participants reported that they had a negative work environment at their respective workplaces. Loud noises, having less support on the job from coworkers and supervisors, getting disrespected by coworkers, and not feeling included in the workplace all contributed to a negative workplace environment. Commenting on work environments, one participant described her experience of feeling excluded at work especially when other coworkers engaged in conversations without her:

"Not really included like, they all sit around and talk to each other, and they don't talk to me, they don't include me in the conversation...I sit with them...I'm just sitting there quiet if I'm there for a few years, yes...it takes a really long time for me to feel included." (\#9 female)

For this participant, it took several years to feel included in a work environment. For participants that are beginning to work or have worked at their place of employment for a relatively short period, it may take time to feel included at work. Feelings of exclusion at work can contribute to a negative work environment, especially if there are no social support systems (e.g., friendly coworker interactions).

Social support systems in the workplace are important, particularly when the work environment is constantly busy and very demanding. For instance, one participant working in a fast-food restaurant was asked if she felt included and respected on the job, she responded with: "Not particularly...it's not a hostile environment, but I don't feel particularly supported 
either" (\#5, female). Later, this participant disclosed that the lack of support stems from exclusion from coworkers and minimal interactions with the manager: "Just like... a lot of them talk amongst each other and I'm not included in that umm...umm it's also like a busy work environment, but also like...my manager never takes the effort to know me..." (\#5, female). Based on these participants' accounts, it seemed common to feel a lack of support on the job from coworkers and managers. Taken together, these experiences suggest that continued exclusion or lack of support on the job can lead to feelings of stress and/or anxiety, especially if participants work in an environment for a prolonged period of time.

\subsubsection{My Positive Workplace Environment}

When participants described why they liked their place of employment, nine participants attributed it to their work environment (e.g., friendly coworkers and employers). Some participants described having a positive relationship with their coworkers and supervisor, which contributed to their positive work environment. Participants also reported feeling fairly welcomed at their place of employment and enjoyed having an environment where coworkers and supervisors facilitated teamwork and collaboration. One participant described how he felt at his place of employment and why he thought it was a great place to work:

"I really like the workplace... the people who work there are really nice... they're really kind to me...they are very knowledgeable about their craft...about their...about the products... umm it's just a really good place to work. It's really the people there that make it a good experience...like is the freedom to ask questions and the environment which is really sort of laid back in that sense." (\#1, male)

This participant commented on the various positive aspects of his workplace, which included his relationship with his coworkers who he described as helpful and knowledgeable and also mentioned how the workplace environment encouraged learning and personal development.

Participants also attributed kind interactions, from coworkers, supervisors and customers, as a reason why they liked their workplace environment. One participant who previously reported a negative experience with her workplace environment (e.g., not feeling supported by coworkers and supervisors on the job) also mentioned that she enjoyed working at a fast-food restaurant because of the kind interactions she had with her customers: 
“Umm...I don't know I just feel like people are generally like pretty friendly and they come up and like...there's definitely been times where like I...made a mistake and gave too much change, especially when I was starting, and they'd be like "oh no you gave me too much change" and I was really happy about that, so it just makes you feel good. Umm or sometimes people would be like "thank you and have a nice day" like I always say to them "thank you and here's your order and have a nice day" and they'd be like “have a nice day”, things that [make me] feel kind of appreciated.” (\#5, female)

This participant commented on her positive work environment and attributed the positivity to the kind interactions she had with her customers on her shift. For this participant, having kind interactions and positive affirmations made her workplace environment more positive in comparison to her perception of a negative work environment due to her coworkers and supervisors. Overall, many participants attributed their positive experience with their employment role to their workplace environment that consisted of friendly and inclusive coworkers and supervisors and kind customers who show their appreciation to employees.

\subsubsection{Challenges with my Coworkers or Supervisor}

Seven out of twelve participants reported having challenges with their coworkers or supervisor. These challenges often related to communication difficulties on the job, which made participants feel unappreciated and undervalued at their place of employment. For example, one participant shared her experience with a certain coworker who continuously ordered her around despite having the same work title at a fast-food restaurant:

"I have a different coworker who always orders me around...I don't know it doesn't say

that she's a supervisor but maybe she's like somehow in charge and she'll be like "[name] do this and do that and do the other thing." (\#5, female)

The participant later described how she felt at that particular moment of being ordered around by her coworker:

"It's kind of frustrating because, like...sometimes it makes me feel kind of insignificant and it just like...can make me feel bad about myself it makes me feel low on the totem pole and like...don't really have the right to be respected..." (\#5, female) 
The comment above illustrates how feeling pushed around by coworkers can make one feel disrespected and unimportant in an employment environment. Negative interactions in an employment setting can also extend to supervisors.

Some participants felt there was a lack of communication with their supervisor, which often resulted in unrealistic expectations of completing an overwhelming amount of work tasks. For other participants, negative interactions occurred when supervisors did not acknowledge employee requests at work. One participant, working in a non-profit organization, recounted a negative experience with his supervisor when he asked to take some time off work due to feeling anxious the day prior:

"Well, the one time that had happened I did, umm, try and tell my employer that I wouldn't be coming in... She was like, "well... if you do feel...we shouldn't try to discount going in to work" and she was trying to get me to go to work because she wanted to keep things regular... and I feel like it was more out of a concern for that than any concern for my well-being...” (\#7, male)

This participant ended up going to work that particular day even though he felt anxious. After discussing the incident, the participant commented on how he felt about the situation itself: “I...didn't feel anxious... that day but I guess, I felt kind of like a doormat for agreeing to go to work when I could have been anxious." (\#7, male)

In relation to negative interactions with supervisors, another participant recounted a time where her supervisor spoke to her in a condescending manner. In this particular incident, the supervisor had plans to terminate the participant's contract through a covert and subtle explanation to the participant. However, when the participant did not understand the explanation, the supervisor pulled out action figures to further explain the situation:

"I feel like you're telling me my contract has to be dissolved and restarted...I don't know what you're saying" and then she pulls out [inaudible] and action figures to explain it to me as if I was a child. So, that was awesome..." (\#9, female)

Participants reported that dealing with negative interactions with coworkers and supervisors can be challenging because it can lead to feelings of frustration, insecurity, and an overall low 
self-worth. When coworkers or supervisors in the work environment create or contribute to a toxic work environment through negative interactions, it can lead to participants feeling stressed and anxious on the job. These results suggest that challenges with coworkers and supervisors can eventually act as stress and/or anxiety inducers in the workplace.

\subsubsection{My Interests and Preferences Align with My Work}

Given the wide range of employment experiences and variation in the types of employment, participants often enjoyed their work because their personal interests and preferences aligned with their employment roles. When participants described the reasons why they enjoyed their work, many reported that they had the opportunity to work in a field that fit with their preference of work. Eight participants reported enjoying their place of employment due to their interests aligning with their work. For participants that preferred not working with customers or people in general, they particularly liked jobs where they worked alone and in solitude. One participant shared this sentiment as he described why he enjoyed his most recent place of employment in comparison to his former job where he dealt with customers on a daily basis: "Enjoyable, since it allowed me to work in an industry that I felt, at the time, suited to my preferences, while allowing me to work mostly behind the scenes and not deal with customer service on a regular basis" (\#2 male). For participants that do not enjoy customer interactions, working in more independent roles allowed participants to enjoy work due to their personal preferences of working alone.

Another participant expressed that he enjoyed his work because he managed to work in a position that aligned with his educational goals:

"I wanted to work something financial something in the business sector because that's sort of the cluster that I'm interested in going into post-secondary...I sort of learn the importance of investing early and using different investment strategies and how some strategies work...I think it's a really interesting field to work in because there's a lot of things about the economy and how to invest and things like that." (\#1, male)

Some participants explained that work was enjoyable because they felt like they personally made a difference at their job. One participant shared her experience: 
"I feel like I'm very fortunate to be with a company that I am at just because I feel like I'm actually making a difference [interviewer says ya]. So, when I feel like I'm actually a part of something and I'm actually not just an ant in a big world [interviewer says ya] ...like I can be replaced like that [snaps fingers] ...I'm able to do stuff like that where it's like actually being a big difference and actually giving my opinion and they're actually going with it and they think they're good ideas ...then it actually pans out." (\#8, female)

This participant described how she felt important, valued, and respected by her employer, which made her enjoy her position even more.

Similarly, one participant working in a software company that tests apps for accessibility expressed a similar opinion when he explained why he enjoyed working at his place of employment: "I feel like I'm doing something good for the disabled community...so that's...so that's something that I look forward to in a job..[interviewer says that's awesome] and ya it can benefit me as well so" (\#3, male). This participant enjoyed working at a company where he knew he was making a difference for those who have accessibility challenges. Being able to work in positions that align with one's interests and preferences and having the ability to make an individual impact at work contributed to this participant's positive work experience. Feeling respected, valued, and included helped participants feel inspired and motivated to continue working and contributed to their positive experiences at their respective places of employment.

\subsubsection{Ways I Prevent Future Stress Occurrences}

After participants described the various stress inducers in the workplace, they often proceeded with discussing ways to prevent future stress occurrences on the job. Participants described their personal methods to prevent certain stress from occurring in their workplace environment. Employees that worked in a workplace environment that required constant communication with customers found ways to prevent stress when dealing with customers at work. One participant described her method of preventing stress on the job by minimizing interactions with customers to the best of her ability: 
"Basically, I just get there, I just stand there, they just come in and bring their stuff and I just check it out...like I noticed usually cashiers are like "hello, how are you and stuff", I just took their stuff, scanned it, asked how would you like to pay and that's the only thing I ever said. So, I figured out how to make it easier for me...minimize what I say...like I did consciously try to come up with ways to minimize my interaction to minimize what I say to people." (\#10, female)

Participants that had challenges with customers on a daily basis resorted to methods such as minimizing interactions as approaches to prevent stress on the job. By minimizing interactions, these participants were able to work without affecting their productivity and also remain stress-free on the job.

For some participants, having an environment where they had full control was extremely vital to prevent stress in the workplace because a few participants in the study reported sensory difficulties in their respective workplace environment. To mitigate and avoid future stress occurrences, one participant described how important it was to have an environment over which she had complete control:

"I completely control the environment. I'm in a portable always so I can always control the temperature. I'm in a portable so I can control the lights, I can control the sound level, I can control everything so that's great...I control the environment right so... and when you're teaching, you have an office, you have the whole room, I control the environment, I don't have any issues there." (\#9, female)

This participant found that it was extremely critical to have an environment where she had complete environmental control because this made her workplace more enjoyable and less stressful.

Other participants mentioned continuously being mindful of taking frequent breaks and knowing their limits with tasks as ways to prevent stress and/or anxiety from the constant work demands. One participant described "chunking" activities together so that each day would be dedicated to a certain activity. Another participant mentioned that they prevented future stress occurrences by figuring out the workload demand before deciding to take a job: 
"When looking at a job opening, I look to see in the job description how much I was expected to do-phrases such as "fast paced environment" and "opportunities for overtime" often let me know that there was an unreasonable workload set out for their employees.” (\#2 male)

This participant described a way to prevent stress in the future by carefully checking the work demands before agreeing to take on a work position. In addition, this participant knew his personal limits and capabilities in a work setting and made sure not to exceed his limitations. In general, participants used personal strategies to prevent a variety of stress circumstances in a workplace setting and by doing so, participants were able to manage and minimize future stress occurrences in the workplace. By understanding stress and/or anxiety triggers in the workplace, these stress preventative measures can be utilized to foster positive workplace experiences. 


\subsection{Theme 2: Stress and/or Anxiety Inducers}

Throughout the interviews, participants shared their personal stress and/or anxiety inducers in the workplace. Despite some differences in the reported situations, participants recounted common experiences with stress and/or anxiety throughout the interviews. This theme comprised six subthemes, each demonstrating various stress and anxiety inducing situations that participants reported. In this specific theme, the first three subthemes were further classified into ASD-related challenges. The theme of stress and/or anxiety inducers begins with the subtheme of "impact of ASD diagnosis on my employment experiences" and in this section, participants voiced their opinions on how their ASD diagnosis negatively affected their work experiences, which often led to feelings of stress and/or anxiety on the job. The second subtheme is "challenges when I communicate or interact at work", where participants commented on their personal experiences with interacting and communicating with people at work (e.g., coworkers, supervisors, or customers) that eventually led to stress and/or anxiety on the job. The third subtheme is "tasks at work", where participants explained how an overwhelming number of tasks can lead to feelings of stress and/or anxiety. The fourth subtheme is "past trauma/experience impacts my ability to work". In this subtheme, participants commented on how their past experiences/trauma affected their employment experiences and ability to work and as a result, led to feelings of stress and/or anxiety on the job. The fifth subtheme of this section is "my stressful experience with disability disclosure", where participants described how disclosing their disability to their respective employers led to feelings of stress and/or anxiety on the job. The final subtheme is the "impact of COVID19", and this is where participants discussed how the COVID-19 pandemic affected their employment status and/or experiences. Together, these subthemes help to conceptualize and convey the personal experiences that participants had with stress and/or anxiety in the workplace. Below, each subtheme is discussed in further detail, using supportive, representative quotations from participants.

\subsubsection{ASD-related Challenges: Impact of ASD Diagnosis on Employment Experiences}

Throughout the interviews, participants often reflected on their ASD diagnosis and how it impacted their professional day-to-day life. Three participants reported that their ASD diagnosis impacted their ability to work. For instance, one participant explained the difficulty 
with having ASD due to the constant meltdowns she had during her stressful work shifts at a grocery store. She commented: "well, basically I was having a meltdown, so the manager took me to his office and he's like, "are you alright, like why are you acting like this?" I was like, "oh it's because I have autism"” (\#10, female). Later on, the participant explained why she had meltdowns at work: "I just could not handle that type of mental work...I was falling apart every few days ... and I was like I can't do this" (\#10, female). This participant described how being employed at the grocery store had a negative impact on her mental health on a daily basis. When asked how she felt about having meltdowns at work, the participant responded with: "it's kind of funny like ...oh, what's wrong with you? Why are you crying like what's wrong with you....? Autism [laughs] so it's like..." (\#10 female). For this participant, meltdowns affected her ability to work as she later mentioned that customers and her manager were concerned with her emotional state at work.

Another participant commented on the impact of ASD on her life as she shared thoughts on why it is impossible for her to work full-time in the future:

"I can't work full-time, like, I physically would not be able to do it, it would just break me down into dust and ultimately that's kind of where I'm at for the rest of my life which is very frustrating because everybody looks at me and goes "oh you're so well spoken, oh you have a degree, oh you must be so high functioning" and I'm like...I'm really not, I'm not that thing and also fuck that term like..." (\#6, female)

This participant described her inability to work full-time due to her personal limitations that resulted from her diagnosis and shared her frustration and stress when people label her as high functioning.

Due to the wide range of characteristics and behaviours with ASD, one participant discussed the challenges she faced when attempting to "appear normal" (i.e., masking) in the workplace. For instance, this participant shared her experience with masking at work and the reason behind why she developed that behaviour:

"I mean, I guess I'm better at masking now and I also further developed the very toxic and unhealthy uhh need to constantly push myself to achieve neurotypical standards even though I logically know that that's not...that's not healthy...” (\#6, female) 
This participant expressed the need to mask to appear "neurotypical" as the diagnosis and disclosure of ASD in the workplace can potentially paint a negative narrative of one's ability and work ethic. For this participant, achieving neurotypical standards by masking can lead to feelings of stress and mental exhaustion.

\subsubsection{ASD-related Challenges: Challenges When I Communicate or Interact at Work}

When participants mentioned stress in the workplace, conversations often led to the topic of communication challenges. These challenges included difficulties with interacting or communicating with customers, coworkers, or supervisors in the workplace, which was the case for eight participants. Concerns regarding challenges with communication were more widespread as participants reported difficulties with thinking quickly on their feet, knowing how to answer questions on the spot, and speaking up more at work. Communicating with people on a daily basis posed a challenge for some participants, especially those that tried to avoid or limit the amount of social interaction as much as possible. For some participants, social interactions were the biggest stress inducers in the workplace. One participant who worked at a grocery store explained how much more she would have enjoyed her job if there were no customers involved:

"Like, not because its human interactions like if my job cuz I like...to take the thing, scan it and put it in the bag, I like that. So, if you could just remove the customer from the equation...like there's nothing wrong with the customers, you're working with the customers, you're going to be fine, but I couldn't handle them." (\#10, female)

The comment above illustrates how customer interactions made the job very difficult and stressful for this participant. Although this participant described nothing wrong with the customers themselves, she found the process of working and interacting with customers on a regular basis very challenging. When asked if these meltdowns were partly attributable to her constant work demands, she said: "absolutely, like I told you, I was so exhausted with the social interactions, my brain was like we have to do something and then I started crying" (\#10, female). 
Similarly, another participant reported feeling stressed when interacting with customers on the job despite enjoying the tasks at work:

"like I could do the job...but it was...it kind of hit a lot of the stress buttons I have, you know, I'm not great with new people, there's like a...ton of new people you have to interact with everyone needs something slightly different, a lot of people had really strange complaints...there was just...I didn't know what to do with this ... you know...it was extremely stressful" (\#12, male).

A common view amongst participants who had difficulties with customer interactions was that they felt stressed due to the constant uncertainty of customer behaviours. In the same vein, participants stated that they personally could not handle interacting with customers on a daily basis for a variety of reasons, one of them being a result of their ASD diagnosis which made it difficult to engage in social interactions. Second, participants mentioned that it was challenging to forcibly interact with a variety of individuals every single day, especially in job roles that consisted of working with customers on a daily basis. In a similar manner, participants expressed feeling stressed and anxious when customers got aggressive, angry, or unreasonable at work. For some participants, they found customer interactions manageable and quite enjoyable, but for many others, these particular interactions on the job led to high stress and anxiety. Stressful interactions were not limited to only customers. For some participants, they mentioned feeling stressed and/or anxious when interacting with coworkers due to the struggles of making friends at work. For instance, one participant, working as a teacher in a school, conveyed her thoughts on how the process of creating friendships at work was always difficult:

"Relationships with other adults are very hard for me because they always look at me weird eventually... Like even when I go into the staffroom whether it's at a school or another company. We're all adults and they'll be talking, and I can never figure out who I'm supposed to talk to and about what and how do you pick who's going to be your friend? Right? Because when you get into that room, other people seem to just pick somebody to be friends with and I don't.... like who do you pick? Then, I end up picking a person that seems nice to me and then I get rude to them and then they don't like [that] 
because I follow them like a lost puppy, but otherwise, how do you get to know somebody?" (\#9, female)

For several participants, building relationships with coworkers was difficult due to the deterring social and facial cues given by coworkers at work. Participants that did not particularly favour social interactions preferred to work independently for long periods of time rather than interacting with customers. Overall, for a majority of participants in this study, they found social interactions and communicating with coworkers, supervisors, and/or customers in the workplace stressful.

\subsubsection{ASD-related Challenges: Tasks at Work}

For many participants, the tasks given at work were reported as stress and/or anxiety inducing, which was the case for nine participants in this study. Concerns were expressed about getting too many tasks at one period of time and being expected to complete these tasks in a short amount of time. Similarly, participants described the stress they felt when given spontaneous tasks in addition to their normal work tasks that needed to be completed in a timely basis. Many participants explained that the tasks themselves were not necessarily difficult, rather the number of tasks (i.e., both general and spontaneous) and the expectation of completing these tasks were stressful. For instance, one participant shared this sentiment when he shared his thoughts on his overwhelming number of tasks at work: "Biggest challenges when working was dealing with an insurmountable workload that I was expected to clear on a daily basis" (\#2, male).

For other participants, being unfamiliar with tasks also initiated stress as participants found it challenging to learn and understand workplace lingo. For example, one participant described how not understanding terminology for one aspect of his task led to a long continuous search for meaning on the internet:

"Umm, I think I can be really thorough in my research which often ends up being a negative because I'll be reading something on the internet, and it'll use a term that I'm unfamiliar with. I'd look up that term and then I'd just go in an endless rabbit hole searching up different terms that I don't understand or different financial instruments that I don't understand." (\#1, male) 
In this case, not knowing the terminology associated with the task eventually made one task into multiple tasks because this participant was not familiar with certain terms. Feelings of unfamiliarity in the workplace made several participants feel overwhelmed and stressed on the job.

For other participants, especially those working with customers on a daily basis, they expressed feeling stressed due to the unpredictability of tasks given each day. For instance, one participant mentioned how difficult it was to work due to the unpredictable nature of their tasks given on a day-to-day basis:

"It's just kind of like the unpredictability of how each day would go...ummm, and just be having to pay attention to so many different people you know... and have to go through so quickly... having the 8 things we had to ask every customer....you know, it was...it was just kind of overwhelming." (\#12, male)

Participants described the stressed, anxious, and overwhelming emotions they felt when tasks were ever changing and too many in number. This was the case for another participant who described the overwhelming number of tasks needed to be completed in a given day:

"At the grocery store, you would get a whole bunch of shipments in, like, you'd get a whole bunch of groceries in, on a single truck. The goal would be to get the stuff done... and get...everything sorted ... and also clean up the shelves at the end of the day as well so...so there are some days where'd you get a whole bunch of stuff at once." (\#4, male)

For participants that had an unmanageable workload, continuous work and playing catchup often led to feelings of stress and anxiety. Although for many participants, the tasks themselves were relatively manageable, the amount of time expected to complete tasks was unmanageable as some participants explained that it took them longer to finish tasks in comparison to other coworkers. For example, one participant commented on the challenges she faced in her place of employment when she needed to complete tasks in a certain period of time: 
"but also like, I'm just not as fast as the others, it just takes me longer to compute like what I'm doing... when it comes to serving the customer it's supposed to be like, take the order and serve it in 30 seconds which I think is crazy, I don't understand how you're supposed to do that" (\#5, female).

For other participants, completing work tasks in a timely manner was difficult due to the outdated instruments and tools provided at their respective places of employment.

"well, the registers were poorly designed...like they would have glitches and breakdowns all the time... so you know it would be like someone's trying to buy $\$ 300$ worth of items and the register freezes" (\#12, male).

In summary, this subtheme of "tasks at work" suggests that when youth and young adults with ASD face challenges related to work tasks, they often feel stressed and/or anxious on the job.

\subsubsection{Past Trauma/Experience Impacts My Ability to Work}

Participants also reported that their individual past trauma and/or experience had an impact on their ability to work, which led to feelings of stress and/or anxiety on the job. This was the case for four participants in this study. For some participants, their past experiences affected their ability to ask for help from coworkers or supervisors on the job, which made it difficult to voice concerns or issues to their current superiors. Other participants described being mistreated by superiors in the past, which made it difficult to have conversations with their current superiors due to social anxiety. One participant reported that he was mistreated by his superiors at school in the past, which affected his self-esteem at his current work position:

"The problems I faced were my own anxiety because asking for things like that can sometimes be really unnerving [interviewer agrees] because you can never know how people react and unfortunately in the past more so with schooling, I had some areas in which superiors, teachers and all that were un-understanding, so that's made me a bit... a bit shy about that at times. " (\#4, male)

For this participant, the past experience of being mistreated by a superior affected his confidence and ability to bring up concerns to his current supervisor. This participant later 
disclosed the challenges he faced when interacting with superiors at work: "I knew my superiors were nice to me... and that a lot of them treated me really well, but because of past experiences, I was scared to ask...you know I was scared I was going to get yelled at or fired” (\#4, male). Even though this participant commented on how kind his current superiors were to him, his past experience/trauma affected his ability to bring up challenges/concerns in the workplace. This participant wanted to reveal his concerns to his supervisor, but due to his past experience/trauma, it made him question his job security.

For another participant, past trauma affected her ability to focus and stay calm during a shift where she thought she saw someone toxic from her past. She explained:

"Other thing I find hard is like umm...uhhh...police officers are bit of a trigger for me [laughs] [interviewer says oh] and so I've had to serve officers in uniform before and that wasn't great. Umm ... and so like there's also someone that I have to avoid contact with and I've definitely thought that I have seen him before and I saw him in line, but it wasn't actually him, it was someone who looked like him [laughs] and I started freaking out [laughs].” (\#5, female)

For participants that worked in the service industry, they were not able to control the environment in which they worked in due to the constant change of customers. This was the case for this particular participant as she needed to serve police officers despite her past trauma, which led to feelings of anxiety and stress on the job.

Some participants also expressed that it took a long time to gain confidence and selfesteem due to their past trauma/experience. This was the case for one participant as she described her experiences with dealing with ASD from a young age and how those experiences impacted her self-esteem as a grown adult:

“My self-esteem was just everything [voice cracks] about being an autistic...you don't make enough eye contact or you make too much eye contact, you don't talk enough or you talk too much they way that you glaze...umm, and every conversation that they have about you, when you're not even in the room with them is about "oh my God, my child won't succeed, my child won't succeed.", (\#6, female) 
Due to her past trauma, this participant had a difficult time describing her current self-esteem in the workplace. This participant's confidence was low from the constant battles she faced in her mind due to her negative past experiences. Negative attributes, labels, and preconceptions of ASD in this participant's mind affected her self-confidence and self-esteem.

\subsubsection{My Stressful Experience with Disability Disclosure}

Participants expressed that their experience with disability disclosure in the workplace incited feelings of stress and/or anxiety in the workplace. For some participants, disability disclosure was an intimidating process because it led to feeling vulnerable. In this study, five participants reported feeling stressed and/or anxious when disclosing their disability at work. Some participants disclosed their disability on the spot due to being put in a stressful situation where they had no choice but to disclose their disability. For instance, one participant revealed that she had no choice but to disclose her disability when her supervisor yelled at her for doing a task incorrectly:

"I did disclose on the spot when my manager got mad at me [laughs].... he was like talking to me...I was trying to explain myself and umm ... because I guess I made a mistake and he tried telling me that I made a mistake, but then I made the same mistake again and he was like "you didn't listen to me!” blah blah blah....I was like "no, I listened I just didn't understand, and I thought I understood! I'm on the autism spectrum, I just didn't know what you meant." ”' (\#5, female)

This participant felt the need to disclose her disability immediately in an attempt to have her supervisor understand why she had challenges completing tasks.

This view was echoed by another participant who described a similar experience when she felt she had no choice but to disclose on the spot to her manager due to her constant meltdowns at her place of employment: "he pulled me over he's like why are you having these meltdowns and I was like I'm autistic, I can't handle social interactions like that..." (\#10, female). This participant also described having a positive experience with disclosing her disability to her employer (e.g., employer was very understanding and accommodating), but particularly the stressful environment led to her disclosing on the spot. Similar to participant 
\#5, this participant did not necessarily choose how she wanted to disclose her disability to her manager.

Another participant described a parallel experience when she disclosed her disability on the spot due to her superior using analogies in attempts to terminate her employment contract:

"I started rocking back and forth... so she came and sat down and explained it and she [principal]goes back to this buffalo analogy and I'm like...so I finally said to her "you know... I have autism, I need you to be really specific and clear and concise because these multiple analogies that you're using...I'm not, I don't know what you're talking about. I don't know if you mean it's my contract you're reducing...", (\#9, female)

This participant felt she was in an uncomfortable position when her principal attempted to terminate her contract in front of her coworkers. Even though this participant did not plan to disclose her disability, she was put in a difficult and stressful situation where she felt she had no choice but to disclose.

Together, these results provide important insights into the process of disability disclosure. For several participants in this study, due to stressful circumstances in the workplace, they felt they had no option but to disclose their disability to their superiors, which led to feelings of stress and anxiety in the workplace.

\subsubsection{Impact of the First Wave of COVID-19}

The global pandemic of COVID-19 affected various aspects of employment for several participants in this study. Specifically, four participants described how COVID-19 impacted their employment experiences. For some participants, the pandemic affected their employment status which either led them to be laid off from work or hindered their job searching process. For one participant who was laid off, he described his plans for advancement in his job role, but due to the pandemic, that was not possible:

"Before the pandemic started, I was working at [place of employment] full-time as a (role), located near [location]. I worked with the company for five months ... but there were plans for me to work much longer than that, with plans for potential advancement, 
pay raises and even promotions, when the pandemic brought down business for the shop.” (\#2, male)

For this participant, there was hope and desire for advancement in his career; however, due to COVID-19 being unexpected, this affected the participant negatively because he was laid off from work.

Due to the sudden nature of COVID-19, several participants were unaware of its potential impact on their employment status. For some participants that were still employed during the pandemic, they reported feeling stressed and overwhelmed due to the health and safety rules and regulations being broken by customers at their place of employment (e.g., wearing a mask). One participant expressed his feelings of stress and anxiety when customers refused to wear masks at a retail store:

"During the middle of the COVID thing it was getting really overwhelming because we were getting a lot of bad customers, like, people who were upset that they had to wear a mask, that sort of thing... and the store owner was like you wear a mask or you don't get to come in...period... We have accommodations if you can't wear it and nobody wanted to take them." (\#12 male)

For participants that worked with customers throughout the pandemic, they mentioned feeling stressed and anxious when customers did not follow government-issued rules and regulations (e.g., physical distancing, wearing face-masks) or acted aggressively or in an unfriendly manner towards employees.

Participants also reported feeling overwhelmed due to the vast changes made at work as a result of the pandemic. For instance, one participant described the changes made to their employment role due to the loss of funding:

“We.... lost funding again ... like every other province funded more for COVID-19, but ours took away funding. We're still (x) millions less last year so when I got to the school, because we have online kids like I should've had a classroom of 24 but a bunch of them went online so when I first got here, it's just (primary) grade and there were 15 kids in my class [interviewer wow] and there were 14 kids in my teaching partner's class and so 
obviously after 2 weeks, we can't have classes this small...like we just can't afford, like..." (\#9, female)

For this participant, COVID-19 affected her employment due to the introduction of online school, which changed the in-person classroom environment. Even though this participant was employed during the pandemic, the environment and stability of her job changed drastically.

\subsubsection{How Stress Affects Me}

When participants spoke about their experiences with stress in the workplace, they often reflected on their emotions and how stress affected their mental health. Eleven participants discussed the negative impact that stress and/or anxiety had on their mental health and employment. For some participants, stress affected their productivity at work, essentially slowing down their pace or being unmotivated to continue with their tasks. For other participants, when confronted with a stressor, they reported getting overwhelmed, sad, and emotional. Similarly, in relation to mental health, participants expressed that stress impacted their self-esteem and self-confidence in their abilities in the workplace. This was the case for one participant as she reflected on her feelings when confronted with a stressor at work:

"aww man...ahh ...like so worthless...this is where the crying starts...its totally okay [tears up] sorry umm...the... it's just a very uhh...intense reminder of all of the crap I went through as a kid for not looking like and sounding like and acting like and being like the other normal kids. Umm...every way that I functioned, was apparently not appropriate and it was always punished whether by bullying or umm..." (\#6, female)

For this participant, challenges and stressors at work negatively affected her mental health to a point where negative memories and emotions surrounded her thought processes.

Several other participants also mentioned the impact that stress had on their mindset and how stress made them question their abilities. One participant described feeling worthless when confronted with stressors in the workplace, which led her to question her ability to work: 
“You know, like I just couldn't do it...it's an I can't do it attitude and I can do it attitude and it's just switching that thought of being like discouraged and you know just feeling like you're not worth it...right? Like you're not important, you're not anything its legit feeling like you're worthless, it's the complete opposite right so... it was hard to get out of that mindset..." (\#8, female)

When confronted with stress and/or anxiety in the workplace, negative and toxic thoughts surrounded this participant's mindset. For other participants, encountering stress on the job on a constant basis led to thoughts about finding ways to avoid work in the future. One participant described a time where he thought of ways to avoid work the following day because of a stressful encounter with a customer:

"When it's really bad, I just kind of shut down...like I can get through it but its slowed way down and it's just kind of like...I don't think I exactly know how to describe it but it's like taking the emotional response and sort of banking it so I won't get in trouble like....so like one person made me want to crash my car coming home so that I'd have an excuse to not come in the next day..." (\#12 male)

Despite shutting down and finding ways to push through the stressors at work, stress negatively impacted this participant's mindset even after his shift. For many participants in this study, stress in the workplace negatively affected their mental health, ability to work, and overall confidence and self-esteem. 


\subsection{Theme 3: Coping Mechanisms}

To overcome feelings of stress and/or anxiety in the workplace, participants shared their personal coping strategies and experiences with coping at work. Most participants reported developing coping mechanisms after experiencing stress and/or anxiety in the workplace. Once participants experienced various stress and anxiety inducers in the workplace, they started to create and employ coping strategies. One participant noted that he needed to experience stress in the workplace to improve his skills at work:

"Ya, and some of that too is also from experience. Like, when I first started out, I was...I would make more careless errors but as time went on, even with the short time I was there at [place A], I felt like I was improving on each day so...I think its lessons that you do learn so you wouldn't make the same dumb mistake again right? So, I was kind of learning by experience if you will in that way." (\#2, male)

This participant described the importance of improvement in his daily work life to overcome struggles and not continue making the same mistakes. By understanding stress and/or anxiety inducers in the workplace on a daily basis, participants knew exactly what to expect and how to respond accordingly.

Several participants highlighted the importance of experiencing high-stress and/or anxiety in the workplace as it helped prepare them for similar experiences in the future. One participant echoed this sentiment when he described his thoughts about bringing in a fidget spinner on stressful days:

"I bring it in like black Friday or boxing day or the days I knew were going to be bad, umm, just so that I would have that there...umm... with most of them, most of the strategies I had was kind of like this is what I'm doing right now...I didn't really...I usually never really planned with a lot of them, I just started using it. ”(\#12, male)

Having experience with stressful days helped this participant prepare and respond accordingly. As a result, participants commented on the importance of dealing with stress and/or anxiety in the workplace to develop coping mechanisms. This is not to say that stress always leads to a positive outcome as demonstrated in the findings from the previous section. 
However, the participants here commented on their experiences with stress and anxiety in the workplace through an alternative perspective.

Throughout the interviews, participants described various coping mechanisms utilized in the workplace. Although there were some differences in the reported coping styles, common techniques used to combat stress and/or anxiety were consistently raised throughout the interview process. Theme three is comprised of three subthemes, each exhibiting the different styles of coping utilized in the workplace. The first subtheme is "problem-focused coping" where participants described their problem-focused coping approaches to overcome challenges in the workplace. The second subtheme is "emotion-focused coping" where participants commented on their various emotion-focused coping styles employed at work. The final subtheme is "pushing through" where participants shared their personal experiences with getting through shifts at work to manage stress and/or anxiety in the workplace. Collectively, these subthemes help to describe and convey the different coping styles used to overcome or manage feelings of stress and/or anxiety in the workplace. Below, each subtheme is exemplified in further detail, using supportive, representative quotations from participants.

\subsubsection{Problem-focused Coping}

When participants spoke on their experience with coping in the workplace, most participants described certain coping styles that would characterize a problem-focused coping approach. For problem-focused coping strategies, they usually involve figuring out the problem, considering potential solutions, weighing pros and cons of the situation, and then selecting a way to approach the situation. In this study, a majority of participants (ten participants) reported using problem-focusing coping approaches to overcome stress and/or anxiety inducers in the workplace. For some participants, when they encountered information or terminology that was unfamiliar to them in the workplace, they used a problem-focused coping approach characterized by researching an unfamiliar topic to get a better understanding of the problem at hand. Doing so often led to a deeper investigation of information through various means of communication (e.g., internet, coworkers, or handbooks), in attempts to learn more about the unfamiliar issue at hand. For other participants, quitting their job was a form of problem-focused coping because they identified 
their workplace as the threat/problem and the only way to overcome that stress inducer was to quit their job.

Although there were some differences in the reported coping styles, participants mentioned common techniques used to combat stress and/or anxiety throughout the interview process. There were 3 common problem-focused coping approaches reported in this study: (1) analyze and solve, (2) asking for help, and (3) writing things down.

Analyze and solve. Six participants reported utilizing problem-focused coping characterized by analyzing the issue and solving an issue at hand. One participant described the process by which he followed to solve an issue at work:

"well, I ask myself, what is the problem, try to boil it down to simpler terms then analyze how the problem occurred. From there, I can then uhh...look at either way to solve it in the immediate or ways to prevent it in the future." (\#4, male)

This participant described his method of solving issues in the workplace by breaking the issue into smaller terms to better understand a problem. By boiling the issue down into simpler terms, this gave the participant the ability to combat an issue and prevent similar barriers in the future.

Asking for help. Four out of ten participants reported using problem-focused coping when they asked for help from a coworker, friend, or family member in attempts to solve an issue at hand. By asking for help, participants reported being able to solve challenges with the assistance from coworkers, family members or friends. One participant reflected on her coping strategies in the workplace with one primary strategy being problem-focused coping because she often sought help from her coworker in times of challenges in order to solve an issue:

"She's not autistic. She'll flat out tell me when it's my fault, sometimes it is my fault and I just don't realize [AV says ya] and sometimes it's not my fault and she'll listen ... at this school, my like my partnering teacher is really super motherly too. So sometimes I'll just ask her "[coworker name], what happened? Can you explain?"," (\#9 female) 
By having coworkers give their perspectives and opinions on issues, participants felt they gained an outside perspective and assistance on how to combat the challenge.

Writing things down. Other participants $(2 / 10)$ expressed that writing things down was another coping style utilized to solve challenges faced in the workplace. By writing things down, participants described being able to tackle down challenges they faced at work into more manageable tasks. One participant described the process and importance of using to-do lists to break down tasks in order to solve a challenge at hand:

“To-do lists [interviewer says oh] ...that...like if I don't have umm...it's really hard to conceptualize a number of tasks to just like as abstract and nebulas and just floating in my head, umm so my brain would just go "HUGE, too many tasks" umm but if I write them down, then they become this concrete thing I can tackle and I also get a much more realistic perception of...how many and how big the tasks are, it always feels bigger before I write it down. Umm like there's a lot of times where people be like "how many things you have to do?" and I haven't written it down and be like "oh my God, probably like 25 " and then if I write them down, I'm like "oh no...this is ...this is 10." ", (\#6 female)

This participant reflected on the value of writing challenges and tasks down on paper because it allowed her to refocus and better manage the stress and anxiety at work. In addition, this participant described the importance of writing things down into smaller tasks as it encouraged her to remain stress-free and solve the issue at hand. In sum, the different types of problem-focused coping approaches utilized by participants in this study allowed participants to help them manage the stress/anxiety faced in the workplace while solving issues at hand.

\subsubsection{Emotion-focused Coping}

Participants reported using emotion-focused coping (i.e., ways to reduce or change negative emotions) when faced with challenges and stress and/or anxiety inducers in the workplace. In this study, all twelve participants reported using a variation of emotion-focused coping approaches in the workplace. Participants reported three common coping styles used to overcome stress and/or anxiety: (1) distractibility, (2) breathing exercises, and (3) social support. 
Distractibility. Eight participants described distractibility as a way to avoid and minimize stress and/or anxiety in the workplace. One participant explained how he tends to be very distractible and loses focus quickly at work and explained why he used distractibility:
"Ya...to stop my mind from working so hard to understand something...to get my mind off of that...the thing that's causing me stress...umm sometimes I will take a detour that sends me off to somewhere else in my mind...other times I'll go to a different activity. " (\#1, male)

This participant used distractibility to deflect negative feelings caused by stress at work. For other participants that used distractibility, they explained that when faced with stressful situations in the workplace, they tend to distract their feelings by focusing on other tasks.

Breathing exercises. Six participants described breathing exercises as ways to minimize feelings of negativity, feel more relaxed, and also focus on something other than the stress at hand. One participant explained how he used a simple breathing technique when confronted with stress and/or anxiety in the workplace:

“Well, I've learned uhh when I'm stressed and anxious, a simple breathing...practices can help me relax a bit...the main one I like to use is belly breathing and its one of those things where you don't exactly have to run off to go do. You can just do it in the moment and help you relax...it's a controlled breathing exercise in which you breathe in through your nose for a set amount of time, hold your breath for a set amount of time, then release for a set amount of time. You then repeat the cycle, and it's a way to both regulate your breathing and you know try to stabilize yourself emotionally and relax yourself enough so you can start to analyze why you're feeling the way you are and you know how you can help work through it because it's really hard to analyze how you're feeling when you're...ragged breath and still caught up in the emotion.” (\#4, male)

For this participant, the purpose of using this specific breathing exercise was to focus on his emotions and breathing in attempts to lower and minimize the feelings of stress in the workplace. Several participants echoed this opinion as they described breathing exercises as ways to prevent anxiety and manage their emotions. 
Social support. Six participants described using social support as a form of coping in the workplace. Social support often included support from coworkers, friends, and family that participants spoke to in times of distress in the workplace. In stress inducing situations, participants often spoke to various members of their support system as a means to release their emotions (e.g., vent or cry) about stressors in the workplace. One participant commented on the impact of social support, especially when faced with stressful circumstances in the workplace:

"hmm...sometimes talking to someone about... how I'm feeling makes it a little easier to bear [interviewer agrees] ...It would usually be [name] sometimes it's been my boss, I've talked to her about how I feel sometimes." (\#7, male)

This participant explained how his social support network consisted of coworkers and mentors in the workplace that he talked to in times of distress. For some participants, having another person to vent or release their emotions can help manage and alleviate feelings of stress and anxiety in the workplace. For example, one participant commented on the impact of having social support in times of stress on the job: “ya ...ya it's good to...ya it's good that they can take some...some weight off" (\#3, male). Talking about negative issues or feelings to a member of a support system minimized feelings of anxiety and stress for participants.

Participants also mentioned other emotion-focused coping approaches. For some participants, they expressed using positive self-talk as an emotion-focused coping approach in the workplace. A few participants reported using journaling as another emotion-focused coping approach in the work environment. Participants used journaling specifically to release feelings and emotions, rather than solving the problem at hand. One participant explained using avoidance as a way to escape feelings of stress and/or anxiety when confronted with challenges that cannot be solved: "I just really avoid it when I can't figure out how to fix something, I just tend to avoid it at all costs" (\#9, female). For this participant, avoiding an issue essentially helped distract feelings of stress, especially when the issue was difficult to solve. In sum, the different types of emotion-focused coping styles utilized by participants in this study allowed them to manage and alleviate the stress/anxiety faced in the workplace. 


\subsubsection{Pushing Through}

Another coping style reported by participants in this study was defined as pushing through. Pushing through can be characterized by participants persevering to move forward at work despite feeling stressed and/or anxious. In this study, nine participants reported pushing through as a coping mechanism when confronted with a stress and/or anxiety inducer in the workplace. For some participants, sticking to a routine and not taking a break were forms of pushing through stress at work. Other participants pushed through their shift because they needed to complete their tasks regardless of how they were feeling. One participant echoed this thought when she described pushing through despite having a meltdown at work: "adapt to be a cashier...I just couldn't so I started having meltdowns...like I tried to power through it ... by the time I realized really something was wrong, I just had to push past and have a new way to look at it" (\#10, female). This participant had a meltdown at work due to the stress of her tasks, and once she realized what was going on, she felt she had no option but to push through. This sentiment was echoed by several participants in this study as they described having no time to cope due to their demanding work roles. Other participants used pushing through as a coping mechanism for getting through their shift at work. For instance, one participant described pushing through as a way to complete his tasks in a two-hour window of work: "I just manage as fast as I can in getting everything done" (\#11, male). Given a time limit of finishing tasks, this participant expressed pushing through tasks as fast as possible in attempts to get through his shift at work.

Participants described not having time to reflect and relax as their work environment was constantly busy, thus they had no choice but to push through their shifts. For instance, one participant working in a grocery store mentioned pushing through as a way to cope during his busy work shifts: "I would just try to power through as fast as I could because well ...it was the only thing I could do at the time right” (\#2, male). For participants working in busy environments, there may be no time to relax or cope on the job and as a result, participants have no choice but to push through the stress and/or anxiety. Another participant mentioned watching the clock as a way to get through a busy shift at work:

"Keeping eye on the time, just counting down which I know it actually makes it feel like way longer... but it was just the ...3 hours left like there's only...like I would really focus 
on that...I'd start kind of ignoring the customers like if people just you know was kind of like shapeless blobs coming up to...like I knew what I had to do.” (\#12, male)

For this participant, pushing through tasks and watching the clock were ways to get through a busy shift at work. Overall, participants often worked in busier work environments where there was no space to relax, unwind, or take breaks from a situation.

\subsubsection{Impact of Coping}

When participants spoke on their experiences with using coping styles, many participants reported the positive benefits with using coping in response to stress and/or anxiety in the workplace. Seven participants discussed the positive impact of utilizing coping mechanisms on the job. For instance, several participants expressed that coping in the workplace helped them refocus their mindset during times of distress and anxiety. One participant explained that coping mechanisms were helpful once they found the right one that worked. Other participants mentioned that coping was beneficial especially when certain stressors occurred on a regular basis, which made the recurring stress and/or anxiety easier to overcome. Participants also described how coping helped improve their mental well-being in the workplace, especially when confronted with stress and/or anxiety. One participant spoke on his experience with using coping mechanisms in the workplace and how employing coping strategies made him feel: "it helps a lot...it puts me in a positive mood regardless" (\#11, male). This participant described coping as a way to get into a positive state of mind in the workplace. Another participant described the positive impact of coping mechanisms at work, especially when dealing with stressful situations with customers:

"Ya, because like you have all the rules of the company and the expectations of the customers and stuff like that ... and then when you talk to your coworkers it's like no this person was actually way out of line, like this customer was acting in a way that was weird or over the top or whatever...like it's kind of the reaffirming that you aren't crazy..." (\#12, male)

For this participant, using social support (e.g., venting to coworkers) as a coping mechanism helped reaffirm his initial emotions and feelings about an unreasonable customer. In this case, 
coping was beneficial to this participant's mental health because he was able to discuss and share his negative experience with his coworkers.

Another participant described the positive impact of coping in the workplace by explaining how coping made him more adaptable to his environment:

“Well, I feel it's helped make it a bit smoother, if I didn't have these coping mechanisms, I wouldn't be able to do my job because one thing could potentially set me off and make me shut down. A person that doesn't know how to cope is a person who doesn't know how to adapt and unfortunately, you know Darwin, that things that can't adapt unfortunately die out, you know survival of the fittest is the survival of those who can adapt and coping mechanisms are you know... mental evolutions way of adapting. " (\#4, male)

This participant described the importance of coping as coping in the workplace helped him adapt to his environment. In addition, this participant highlighted the overall importance of being adaptable, especially in a competitive workplace environment.

Despite most participants describing positive impacts to coping, there may be negative consequences to coping in the workplace, especially when employing coping strategies such as pushing through. For one participant, pushing through the situation eventually led to a meltdown in the workplace. Therefore, pushing through may not necessarily be a positive impact of coping. On the other hand, most participants described problem and/or emotionfocused coping in the workplace as a way to manage stress and/or anxiety in the workplace because it helped their mental health, made their workplace tasks more manageable, and boosted their overall mood. 


\section{Chapter 5}

\section{Discussion}

Gaining employment is often associated with a sense of purpose, quality of life and financial independence, and functions as an indicator of adult success for both the general population and for people with ASD [18,23]. Despite youth and young adults with ASD having the motivation and skillset to work, they continue to experience many challenges in maintaining employment [62,119]. For many people with ASD, stress often poses as a threat in the workplace due to challenges such as interpersonal relationships and sensory sensitivity, $[29,115]$. There is a paucity of literature exploring the experiences of youth and young adults with ASD and how they cope with work-related stress and anxiety on the job [29]. Examining coping mechanisms in the workplace is a novel area of research because it focuses on individual experiences of addressing work-related stress and/or anxiety, which can potentially point to reasons for maintaining employment. Up until now, the majority of studies on employment outcomes for youth and young adults with ASD focus on securing employment with less emphasis on how they maintain their employment. This study is one of few qualitative studies that explores individual, direct experiences of youth and young adults with ASD as they manage stress and/or anxiety in the workplace by using their personal coping mechanisms. It is critical to explore this topic because it provides insight into potential strategies that can be used to manage feelings of stress and anxiety in the workplace. This insight can also be useful for employers who are looking to hire inclusively and wanting to know how they can better support employees on the job.

This current study builds upon previous research on employment experiences for people with ASD [26,30]. The focus here, however, was on the individual experiences of youth and young adults with ASD with coping in the workplace in response to work-related stress and anxiety. It is also, to my knowledge, one of few studies to explore both the types of stress and/or anxiety inducers in the workplace and coping styles in response to work-related stress and anxiety among youth and young adults with ASD. Exploring this area of research is important given that coping mechanisms can be utilized to manage and alleviate feelings of stress and anxiety in the workplace. In the following section, I present a discussion of the 
findings followed by implications for employers, coworkers, people with ASD, and clinicians, limitations, and conclude with future directions.

\subsection{Discussion of the Findings}

The overall objectives of this study were to explore the stress and/or anxiety inducers in the workplace and to explore the coping mechanisms utilized in the workplace among youth and young adults with ASD in competitive employment. Through IPA, I found several themes that shaped participant's employment experiences related to stress and/or anxiety in the workplace. These themes were characterized by categories including salient workplace factors that can contribute to the overall positive or negative work-related experiences, specific stress and/or anxiety inducing situations in the workplace, and individual coping mechanisms reported in the interviews. Although these themes emerged and participants had similar experiences, it is important to acknowledge that each participant's experiences were unique, which highlights the overall nature of phenomenology and also underscores the individual and varied nature of ASD as it is a spectrum of experiences. Therefore, some coping strategies or positive work experiences/supports described by one individual may have had the opposite effect for others.

In addition, although gendered experiences were identified in the interview scripts, I found that there were no gender differences among the identified themes and subthemes. For instance, when I was analyzing the data set, I did not find that males encountered a certain stress or employed a certain coping mechanism; rather, both males and females had similar experiences in the workplace and responses in the interviews.

For many of the subthemes, experiences were further classified as either positive or negative. When participants talked about disclosing a disability, asking for adjustments and their workplace environment, participants had either positive and/or negative experiences regarding each respective subtheme. Hence, I wanted to highlight the fact that although elements in the workplace may be similar (e.g., workplace environment), the experiences themselves truly vary between situation, context and the individual. Upon deeper reflection, I believe that my experiences as a job coach impacted the way I viewed and characterized the dataset (e.g., positive and negative experiences with disability disclosure). Having job coached many individuals with intellectual disabilities, I know my experiences and my own 
personal thoughts played a vital role in this research study as I was made more aware and connected to the work related challenges and stressors brought up during the interviews.

The following sections contain a discussion of how my study findings align with and extend previous research on employment experiences among youth and young adults with ASD, followed by a discussion of the results in relation to the theoretical framework.

\subsection{Salient Workplace Factors}

\subsubsection{Disclosing a Disability in the Workplace}

Participants in this study highlighted the potential risks to disability disclosure in the workplace. Disclosing a disability at work can be difficult because it puts individuals in a vulnerable position. These findings are supported by disability disclosure literature among young adults with ASD who discuss the risks to disclose their disability at work [33,44]. For example, in this study, participants described risks to disclosure such as fear of discrimination and/or judgement and self-conscious insecurity. Fear of discrimination often poses a threat in disability disclosure, specifically if youth and young adults with ASD have been discriminated against in the past for disclosure [191]. Youth and young adults with ASD may be scared to disclose their disability at work due to negative attitudes and misconceptions about ASD, which can potentially contribute to ASD being invalidated by an employer [191]. Disclosing a disability can be difficult for people with ASD because they may be weary of the stigma and lack of understanding of ASD in the workplace, which has been identified in the employment literature as having a significant impact on an individual's ability to retain employment $[74,145,192,193]$. This study suggests that stigma associated with having a disability may prevent individuals from disclosing their disability out of fear of losing their job or being treated unfavourably at work [194]. The results are also consistent with those of Lindsay et al. who found that youth with various disability types (e.g., cerebral palsy and muscular dystrophy) expressed a variety of barriers to disclosing a disability in the workplace [175]. Barriers to disclosing a disability included fear of stigma and discrimination from employers, negative past disclosure experiences, and lack of employer's knowledge of disabilities and adjustments in the workplace [175,195-198]. 
Barriers to disclosing a disability in the workplace can be linked to the visibility of the disability. For instance, this study show that many participants were hesitant in disclosing their disability because there are risks to disclosing a disability, especially when the disability is not visible. Studies show that those with visible disabilities (e.g., physical disability) often feel as though there is no option but to disclose their disability to their employer $[197,199]$. People with invisible disabilities such as ASD may be more hesitant to disclose their disability due to fear of stigma and discrimination [200], which was the case in this study. For employers, it may be easier to identify and accommodate individuals with a physical disability in comparison to those with a less visible/invisible disability such as ASD. However, it must be noted that every individual deserves adjustments and accommodations in the workplace if they are needed.

On the other hand, several participants recalled a more positive experience with disclosing a disability at work. When participants disclosed their disability, many reported feeling relieved and more confident in the workplace. Participants also highlighted the importance of building a trusting relationship with their employer in order to feel more comfortable with disclosing a disability at work. Employers play an important role in cultivating an environment that make individuals with ASD feel more included and valued in the workplace [69]. Studies indicate that positive relationships with employers can promote individuals with disabilities to disclose their disability and ask for accommodations in the workplace [196,201-203]. Similarly, positive attitudes towards people with disabilities in the workplace can facilitate positive employment experiences for youth and young adults with disabilities. Studies have indicated the importance of employers creating a workplace culture that is healthy and safe for employees [204,205]. These findings are also evident in this study where participants described the value and positive experiences with disclosing a disability when the workplace culture was safe and supportive. Whether the relationship is with a coworkers or supervisor, fostering a healthy and positive workplace environment entailing positive relationships helped participants feel more included, comfortable, and overall happy in the workplace.

In accordance with the present results, previous studies found that it is vital for employers and coworkers to create a workplace environment that is inclusive and supportive of diversity through workplace practices and initiatives [205,206]. Having an environment that empowers 
people with disabilities in the workplace allows participants to be themselves and thrive [207]. For example, one study that explored a work experience program for autistic adults at a bank in the UK found key challenges that stemmed from environmental or situational factors that contributed to participants' social and communication difficulties on the job (e.g., knowing how to ask for help) [205,208]. In the case of the participants in this study, their experiences demonstrate the critical need for employers to foster an environment that can allow participants to ask for help or raise concerns. These experiences underscore the importance of environmental elements (i.e., employers and coworkers), which can foster or hinder an individual's success in the workplace.

The results from this study demonstrate that the process of disability disclosure is personal and voluntary, and much thought often goes into the decision on whether to disclose or not. Upon deeper reflection, the results from this study show how disclosing a disability can be challenging and daunting for some individuals and/or liberating for others. From discussions related to potential risks, participants described a variety of reasons as to why disclosing a disability in the workplace can pose a risk to their employment. Many of these reasons surrounded issues related to the environment and how others in the workplace (e.g., coworkers and supervisors) would react and act towards employees with ASD. While seemingly in opposition, participants who described a more positive experience with disclosing a disability in the workplace often attributed these positive experiences to their environment as well. Specifically, these participants described having a positive relationship with their employers and explained how employers who have inclusively hired in the past, essentially made the process of disclosing a lot easier. These findings help us understand the various experiences of disclosing a disability in the workplace and how there are certain aspects of the workplace environment that can contribute to this overall positive or negative experience. Moreover, these results demonstrate how aspects of the workplace environment that can be a barrier or facilitator in the workplace.

\subsubsection{Asking for Adjustments in the Workplace}

Participants described their negative experiences with asking for workplace adjustments at their respective place of employment. Similar to other ASD-related studies in the literature, participants in this study asked for consistent scheduling, an option to work from home, and 
adjustments to the job tasks $[33,175,209]$. However, for some participants in this study, employers refused or did not take these requests made by employees in this study very seriously. Similar to disclosing a disability in the workplace, asking for adjustments and accommodations can be a challenge that people with disabilities including ASD encounter in the workplace [200,210]. Consistent with the findings of this study, other studies have reported that people with disabilities (i.e., physical and mental) face barriers to employment (i.e., maintaining employment) as a result of unmet adjustments and accommodation needs or refusal $[211,212]$. Employers may unintentionally implement a broad approach to disability in the workplace, with limited knowledge of ASD and the unique adjustments and needs required by people with ASD $[99,145]$. Hence, individual needs and adjustments may go unnoticed by employers. For youth and young adults with ASD in this study, this may be frustrating and confusing, especially when adjustments are not addressed by employers who may not know how intimidating it is to ask for adjustments on the job. Another important finding was that for one youth in this study, bringing up concerns related to adjustments on the job meant asking for a different job role because adjustments on the job were not possible. Although youth and young adults with ASD are capable of working, adjustments on the job can potentially make the tasks and role itself less stressful and more manageable [213]. Hence, it is important for employers to both know and understand the needs of people with ASD in the workplace and how workplace adjustments may vary by individual $[99,214]$. These results suggest that if adjustments to the job are made possible for youth and young adults with ASD, it may open up more avenues for paid employment and prevent workrelated stress and/or anxiety in the future.

Nonetheless, when participants spoke on their experiences with being employed, many described their positive experiences with asking for workplace adjustments. The results from this study show that positive experiences with asking for workplace adjustments essentially reflect the importance of employer knowledge, empathy, and workplace flexibility for employees with ASD. Studies highlight the critical need for employers to be more inclusive, empathetic, and flexible to adapt to the overall work environment and work demands to better accommodate people with disabilities [205,215]. This study agreed with those findings as employers that were more informed of ASD in the workplace worked with the participant's needs and strengths in the workplace (e.g., participant that used a summary sheet to show 
strengths to employer). This finding is valuable because it demonstrates that by giving individuals with ASD space to grow and succeed in their place of employment, they are able to showcase their strengths and abilities on the job. Throughout the interviews, participants also noted the importance of having employers set clear expectations and rules. When participants described negative experiences in the workplace, they often attributed challenges to unclear expectations. If workplace tasks and expectations are more blurred and undefined, it may discourage and demotivate participants to work to their full potential [216]. A supportive employer may be more inclined to modify work tasks or the environment, adjust communication, and help with social interactions, which can help with employee retention [194]. These are clear ways to encourage employment among youth and young adults with ASD. The role of the employer plays a large role in an employment setting and if employers build better communication and rapport with their employees, it may facilitate open conversation about accommodations on the job for people with disabilities [175].

The results from this study show both positive and negative experiences associated with asking for adjustments on the job. Some of the issues emerging from these varying experiences relate specifically to employer awareness and understanding of ASD in the workplace. Due to the heterogeneous nature of ASD, individuals cannot be placed in rigid categories, and this highlights that accommodations and adjustments are not a "one-size-fits all'. Thus, it is vital for employers to understand the working capacity and limitations of their employees with ASD to better facilitate a comfortable working environment

\subsubsection{My Workplace Environment}

For youth and young adults with ASD in an employment setting, it is important to have a work environment that is supportive and welcoming by both supervisors and coworkers because it can lead to better employment outcomes [194]. Several participants in this study described working in a negative work environment, which made them feel excluded and not supported on the job by supervisors and/or coworkers. For adults with ASD to be successful in the workplace, it is important for employers and superiors in the workplace to provide a supportive and understanding work environment [116,217]. Continuous negative feelings on the job can potentially lead to youth and young adults with ASD feeling stressed and anxious 
on the job. The findings of this study underscore the need to promote a supportive workplace environment where individuals with ASD feel included, respected, and valued.

On the other hand, the work environment could act as a facilitator in the workplace that can make participants' experiences in the workplace more positive. The majority of participants attributed their positive experiences in the workplace to their overall work environment. The positive work environment often entailed encouraging and optimistic coworkers and supervisors who helped foster diversity and inclusion in the workplace. The results, which underline the value of the work environment that can either hinder or facilitate the inclusion of people with ASD in the employment market, further supports previous studies [26,218,219]. The work environment plays a large role in success among youth and young adults with ASD because the environment itself can induce feelings of stress and anxiety as shown through the results of this study. For some participants in this study, the ability to control their work environment (e.g., temperature and sound) contributed to their positive experiences and lower stress levels. Studies highlight the fact that struggling to manage and cope with sensory stimuli can lead to anxiety along with challenges with social interactions and being able to withstand unpredictable changes in situations [18,76,220].

Employers also play an integral role in helping youth and young adults with ASD succeed in a work environment [116]. For employees with ASD to be successful in an employment setting, it is important to have a supportive and welcoming environment expressed by coworkers and supervisors because it is associated with better employment outcomes for employees with ASD [194]. The results highlight the importance of employer knowledge and understanding of disabilities in the workplace to help facilitate a positive workplace experience for youth and young adults with ASD. Youth with disabilities are often more comfortable working in an environment where they are supported by employers who are understanding, non-judgmental, and overall respectful in the workplace $[175,195]$.

In many circumstances, workplace social challenges are often attributed to employees with ASD rather than a reflection on workplace or societal elements (e.g., lack of support on the job or existing workplace policies) that could be problematic and of need of modification [137]. This study also highlighted the importance of supervisors adjusting to make the workplace environment less challenging for employees with ASD including educational 
strategies for coworkers and employers (e.g., giving clear instructions to employees with ASD) and developing protocols and resources for employees with ASD on how to handle workplace challenges (e.g., self-care strategies) [137]. It can be suggested that it is not wholly the responsibility of an employee with ASD to acclimatize to the work environment, rather, the work environment (e.g., coworkers and supervisors) can change to be more inclusive and supportive of the needs of employees with ASD [77].

Overall, the results from this study show both positive and negative elements of a workplace environment that can impact an individual's experience in the workplace. Taken together, the findings indicate a critical role the workplace culture and environment play on the overall employment experiences of employees with ASD. For participants that described a more negative workplace environment, they attributed these negative experiences to feeling unsupported or as one participant mentioned, feeling excluded from conversations in the workplace. On the other hand, for participants that described a positive workplace environment, they often attributed these positive experiences to the workplace culture (e.g., supportive coworkers and supervisors). The results underscore the role of the workplace environment which can facilitate an individual's integration into the employment setting. Thus, the findings show that supervisors and coworkers can play a tremendous role in creating a friendly and welcoming space for individuals with ASD.

\subsubsection{Challenges with my coworkers and supervisors}

When participants spoke about their workplace experiences, many reported on the challenges they encountered in their respective place of employment. A majority of participants described having challenges with their coworkers and supervisors at work. Challenges ranged from communication difficulties in the workplace to negative interactions with coworkers and supervisors. These results are consistent with previous studies on the barriers to employment success for youth with ASD $[11,26,28]$. In this study, participants also expressed the difficulty with interpreting unclear and implicit language in the workplace from their superiors, which made their respective roles in the workplace challenging. In accordance with the results, previous studies demonstrate that the inability to interpret other people's intentions and emotions are common challenges in social communication among people with ASD [221,222]. Supervisors and colleagues often describe social challenges for adults with 
ASD including being social at an inappropriate time, not being able to follow implicit rules in a conversation or having problems interpreting non-verbal cues $[46,61,72,137]$.

Communication difficulties in the workplace can negatively impact one's employment status as shown through the case of participant \#9 when their supervisor spoke to them in a condescending tone in attempts to terminate their contract on the spot. In this particular situation, the supervisor used action figures to further explain the termination of the participant's contract because the participant was not understanding the implicit language being used. There is a lot to unpack with this situation. For instance, this circumstance highlights the importance of workplace culture and having supervisors treat employees with respect and dignity. Even if a contract needs to be dissolved or terminated, the supervisor has the responsibility to deliver this information in a respectful and understanding manner. In the same vein, this situation underscores the value of explicit language in the workplace, especially when sensitive topics such as contract terminations are discussed. Hence, these findings suggest that if implicit and unclear language continue to be used in the workplace, it can pose as a potential stress and/or anxiety inducer in the workplace for youth and young adults with ASD, which can impact one's ability to remain employed.

\subsubsection{My Interests and Preferences Align with My Work and Personal Strategies to Prevent Future Stress Occurrences}

Participants also attributed a positive experience in their respective workplace when they worked in a field that aligned with their interests. Throughout the interviews, a majority of participants described the joy and happiness they felt when working in a role that fit with their interests and personal preferences. In regard to personal limitations, several participants expressed the value of working in a role that minimized social interactions. Hence, for individuals that prefer working alone with minimal interactions with customers and/or coworkers, finding a role that limits interactions can prevent future occurrences of stress and/or anxiety on the job. This point can be further explained by the results of this study as participants that did not enjoy social communication or interaction on the job often recalled positive experiences when they were able to work in a more independent role. Studies have also highlighted the importance of focusing on strengths and matching skills, interests, and abilities to the job role in order to maintain employment for adults with ASD [30,74]. By focusing on individual strengths and skills, employers can allow youth and young adults with 
ASD to engage in the workplace. Participants also expressed the importance of working in a field where they could personally make a difference to the work environment. These findings suggest that employment can provide an opportunity for adults with ASD to flourish in their specific interests and can benefit both themselves and society [21].

Throughout the interviews, participants described several stress preventative measures to facilitate better workplace experiences and limit stress occurrences on the job. These stress preventive measures were unique because they ranged from techniques utilized before gaining employment (e.g., looking at the job description before taking on the role) to techniques used on the job (e.g., minimizing social interactions). In one study, employees with ASD reported approaches to prevent social challenges in the workplace including self-directed strategies (e.g., getting help from a professional to manage feelings of anxiety) and making personal adjustments (e.g., determining personal strategies to prevent reoccurring social challenges in the future) [137]. These results parallel the findings in this study where some participants avoided/minimized social interactions in the workplace to the best of their ability to prevent stress and/or anxiety in the workplace. These findings suggest that avoiding workplace environments known to incite feelings of stress and tailoring to individual needs can improve employment outcomes for people with ASD.

In this study, participants highlighted the significance of looking at the job description/work environment before committing to a job role. One suggestion for employers includes making job postings detailed and explicit so people with and without ASD know what to expect in the job role. It is possible, therefore, that knowing the work environment before taking on the job role can prevent stress and/or anxiety on the job. Unlike coping mechanisms that are usually employed during a stressful situation, stress preventative measures can be used to avoid stressful encounters in the workplace.

Overall, these subthemes highlight the fact that these workplace factors can facilitate positive workplace experiences for youth and young adults with ASD. However, the results from this study also demonstrate the connections between themes as several negative aspects in the workplace (e.g., disability disclosure, asking for accommodations, and workplace environment) can eventually lead to stress and/or anxiety on the job. The interrelation between these themes revealed that context, situation and the environment truly play a role in 
the workplace and how the combination of these factors can affect an individual's experience at work. Despite some aspects of the workplace environment being similar (e.g., process of disclosing a disability), the context and situation in which they occur can lead to these experiences being stress and/or anxiety inducing for youth and young adults with ASD.

\subsection{Stress and/or Anxiety Inducing Situations in the Workplace \\ 5.3.1 ASD-related Stress and/or Anxiety Inducers: The Impact of My ASD Diagnosis at Work}

Employment for youth and young adults with ASD can be challenging due to inherent characteristics of ASD that may affect employment experiences. Participants recalled having meltdowns, knowing their personal limitations for taking on a larger workload, and masking in the workplace to appear more neurotypical. For people with ASD, interactions and social communication may induce feelings of stress and/or anxiety on the job. If a job role involves high levels of social interaction (e.g., service sector roles), there may not be an option to alter the work tasks. In accordance with the present findings, previous studies have demonstrated that adults with ASD who struggle with social communication may experience negative feelings such as meltdowns, which may affect job performance [137,223]. Other research shows that meltdowns could also be a result of a more sensitive reaction to an environmental stimuli that an individual cannot tune out [224]. A possible explanation for meltdowns might also be that youth and young adults with ASD who struggle with social communication challenges may also struggle with asking for help from their employers to readjust or modify tasks, which can ultimately cause meltdowns in the workplace [29]. However, it is also important to consider the environmental and situational elements (i.e., workplace environment, coworkers, or customers), which can potentially result in circumstances that could influence stress and frustration in an employment setting. Thus, there are a myriad of factors that can impact an individual's response to stress on the job.

The concept of masking also came up during the data collection process of this study. In the context of this study, masking was used to appear more "neurotypical" in the workplace as the diagnosis of ASD can paint a negative view on one's ability to work. People with ASD who resort to masking often do so in an attempt to appear more neurotypical and to avoid awkward social communication (e.g., talking too much) in the workplace [143]. However, 
masking can put an emotional strain on people with ASD as it can lead to feelings of stress on the job [191]. In the literature, masking is typically utilized by females with ASD in the workplace as it is this group that has the ability to identify and understand how to disguise their differences and conceal their inherent challenges with social communication through learned behaviour $[53,225,226]$. One female participant in this study used masking to appear more neurotypical in the workplace; this finding therefore needs to be interpreted with caution because it cannot be generalizable to all females with ASD.

\subsubsection{ASD-related Stress and/or Anxiety Inducers: Challenges When I Communicate in the Workplace}

For most youth and young adults with ASD in this study, they described feeling stressed and/or anxious at work, especially when it came to communicating with customers, coworkers, and/or supervisors. In the area of employment, the unique characteristics of ASD often manifest in difficulties with successfully communicating and integrating with coworkers in the workplace [28]. In a similar manner, autism is often characterized by difficulties in navigating social scenarios [28]. For adults with ASD who are employed, communication difficulties are often found to be the most challenging barrier to maintaining employment $[62,74]$. Dealing with customers and the unpredictability of their moods and attitudes contributed to feelings of stress and/or anxiety for some participants in this study. These findings are in accordance with other studies which found social challenges (i.e., communication difficulties in the workplace) being frequently associated with emotional responses (e.g., stress and anxiety) for people with ASD [18,137,191]. For adults with ASD in an employment setting, difficulties with understanding informal workplace conversations, reading facial expressions, and understanding tone of voice are among the common challenges to navigating social interactions in the workplace [28].

In a work environment, the bonds created between coworkers can be strong enough to lead to long-term friendship, and by having these emotional and social supports on the job, it may help alleviate isolation and loneliness experienced by individuals with ASD [194]. In this study, participants often attributed challenges with communication to an internal issue (i.e., place blame on themselves), rather than the environment (i.e., neurotypical social norms). There are similarities between the attitudes expressed by participants in this study and those described by Bury et al. in a study that uncovered social challenges in the workplace among 
adults with ASD [137]. Bury et al. found that adults with ASD typically attributed social challenges to factors related to the person with ASD rather than a criticism on the broader work environment or general societal norms [137]. The blame is often placed on individuals with ASD rather than critiquing the potentially problematic ways in which coworkers and supervisors interact and communicate with people with ASD and the broader environment. On the other hand, studies found that this challenge goes both ways as neurotypical people also have difficulties understanding how people with ASD think and feel [227,228]. This problem can be characterized as the double empathy problem which suggests that when people with varying experiences of the world interact with one another, they will have challenges empathizing with one another [228]. As a result, these varying differences may lead to a breakdown in communication that can be stressful for both people with and without ASD [227]. These communication barriers on both ends can make it challenging for people with and without ASD to effectively share experiences and connect with one another [227].

\subsubsection{ASD-related Stress and/or Anxiety Inducers: Tasks at Work}

When participants spoke about their experiences with feeling stressed and/or anxious at work, they often blamed the tasks in the workplace for inciting these feelings. For several participants, the tasks themselves were not difficult to complete, rather the unpredictability and unclear expectations of tasks in a given day contributed to stress and/or anxiety on the job. Consistent with the literature, this research found that participants had difficulties multitasking in the workplace $[26,229,230]$. Many individuals with ASD need to have a clear understanding of what is expected of them in regard to job productivity and types of tasks given $[18,218]$. Upon deeper reflection, it appeared that the volume of tasks may have heightened the stress and anxiety that participants felt on the job. In addition, due to the busy nature of the workplace environment, there was a lack of structure or organization aligned with the types of tasks that were given to these employees with ASD, which also often induced stress and anxiety on the job. The findings from this study highlight the need to educate and inform employers and coworkers of the capabilities and limitations of individuals with ASD in the workplace. If youth and young adults with ASD continue working without a clear set of tasks and expectations, they may continue to feel stressed and/or anxious on the job [231]. 
In the intellectual disabilities (ID) literature focusing on employment, workers with ID who perceive work tasks more intensely also experience high levels of job stress, which negatively impact productivity and quality of life for employees [232,233]. The literature shows that employees with ASD succeed in employment when managers and superiors adopt clear and explicit communication styles in the workplace [116]. Employers need to be attuned to the needs of explicit communication to help create a manageable and successful work environment for employees with ASD [234]. Supervisors and other leaders in the workplace should give encouragement, set goals for tasks, and help coordinate work activities because employees with ASD are more likely to respond favorably to superiors who provide them with support [235]. More importantly, when employees with ASD are faced with a challenging task, it is important for leaders in the workplace (e.g., supervisors) to recognize the issue and provide necessary supports to help ease any feelings of anxiety that may arise [235].

\subsubsection{My Past Traumas/Experiences Impact My Ability to Work}

Participants also discussed their past traumas/experiences that led to feelings of stress and/or anxiety on the job. Participants described being mistreated in the past, dealing with triggers in the workplace, and their personal experiences with being autistic. In general, stressful and traumatic events are known to increase risk for mental health outcomes [236238]. This outcome is no different for people with ASD $[113,239]$. The literature shows that people with ASD are at higher risk for experiencing stressful and traumatic life events, which can negatively impact mental health $[239,240]$. Participants that dealt with their past trauma on the job often experienced ongoing stress and anxiety. These feelings of stress and anxiety seemed to have affected participants' self-esteem and confidence on the job. For other participants, they reported that their past trauma occupied their mental space for the duration of their shift (i.e., making them constantly focus on the stressor), which led them to feel stressed and anxious. Thus, in the context of employment, past trauma and experiences seem to induce feelings of stress and anxiety in the workplace. Although there seems to be a paucity of literature regarding the relationship between trauma and past experiences and employment for youth and young adults with ASD, results from this study indicate the negative relationship that trauma and negative experiences have on one's employment. 


\subsubsection{My Stressful Experience with Disclosing a Disability at Work}

Participants in this study also spoke on their own personal experiences with disclosing a disability in the workplace. For several participants in this study, disclosing a disability at work induced feelings of stress and/or anxiety on the job. Most of the participants that described a negative experience actually had similar experiences with disclosing their disability on the spot due to feeling stressed on the job and being placed in an unpredictable circumstance. Studies show that youth with less-visible conditions (e.g., mental illness, arthritis, and other various conditions) are more likely concerned about the stigma and reactions from others, which can hinder them from disclosing a disability at work [201,211]. Employees with a disability may decide to disclose a disability when they feel that they no longer have the capacity to mask their disability (e.g., as a consequence of stressful life events) or if they experience a negative disability-associated event in the workplace that needs to be further explained to avoid negative repercussions from employers [214,241,242]. For participants in this study, stressful situations (e.g., meltdowns, getting yelled at by a supervisor, and employer attempting to terminate employee contract) forced them to disclose their disability on the spot. For these participants, disclosing their disability was a reaction to their stressful situation, rather it being a premeditated decision. Results from this study provide personal experiences in disclosing a disability on the spot, especially in a worksetting, which is currently lacking in the literature.

\subsubsection{How COVID-19 Affected My Employment Experiences}

The COVID-19 pandemic also impacted employment experiences for participants in this study. Due to the pandemic, participants faced unemployment and overall drastic changes in their respective workplace environment. Around the world, people encountered challenges maintaining employment due to the drastic changes in the work environment and overall public health concerns [243,244]. In general, individuals faced drastic changes in work practices (e.g., working from home, virtual communication) and behaviours (e.g., social distancing) during the pandemic [244]. Similarly, people with ASD often dealt with many challenges during the pandemic including managing uncertainty, adapting to changes in schedules, and dealing with long periods of social isolation [149]. One study also suggested that the global economic crisis caused by COVID-19 negatively affected employment stability for adults with ASD [147]. These findings reflect the results from this study as participants 
described difficulties with job security due to the pandemic. In accordance with the present results, previous studies have demonstrated that participants who were employed during the peak of the first wave of the COVID-19 pandemic faced challenges including increased work demand and extreme alterations of working conditions and environments [243,245]. Regardless of COVID-19, employment outcomes for individuals with ASD in general are relatively poor with high rates of unemployment [97]. Thus, with the added stressor of a global pandemic, this can make individuals with ASD particularly vulnerable to stress [246].

In relation to the pandemic, many participants in this study did not have an option to work from home. For those that described COVID-19 as a stress and/or anxiety inducer, these participants all worked in-person. Hence, feelings of stress and/or anxiety on the job could be explained, in part, by the inflexibility to work from home, which could help to eliminate sensory needs, lower risk of COVID-19 infections, and reduce commute times [246]. Participants also recalled feeling stressed and anxious when customers were reluctant to follow rules and government-issued health mandates in the workplace. Therefore, with the constant pressure and stress with social interactions with customers in the workplace, there was often an added stressor of customers being aggressive and hostile towards participants that worked in the service sector industry.

Overall, these subthemes help to demonstrate the types of stress and/or anxiety inducing situations that participants experienced on the job. Similar to the previous theme, the findings from this theme reveal the connections between subthemes. In particular, several subthemes from the previous section (i.e., disclosing a disability and communication challenges in the workplace) were indeed stress and/or anxiety inducing for participants in this study, which emphasizes the intersectionality and transactional nature of these themes.

\subsection{Coping mechanisms}

\subsubsection{Problem-Focused Coping and Emotion-Focused Coping Strategies}

Most participants described using problem-focused coping in response to feelings of stress and/or anxiety in the workplace. The most common problem-focused strategies reported in this study included analyzing the situation and solving the issue at hand, asking for help to solve a situation, and writing things down. All participants in this study also described using a 
form of emotion-focused coping in the workplace, which included common strategies such as distractibility, breathing techniques, and social support.

There is limited literature that looks at coping strategies in the context of employment for youth, young adults, and adults with ASD in the literature. However, there are studies that discuss coping in relation to everyday activities (e.g., everyday life and school-related experiences). For instance, one study looking at university experiences of students with ASD found that students coped by knowing their limitations (e.g., part-time enrolment in school) [247]. Similar to knowing one's limitations as a form of coping, in this study, quitting a job related to a form of problem-focused coping. Specifically, in this case, the participant in this study knew their personal limitations and in order to avoid feeling stressed and anxious on the job, they acknowledged their personal limitations and decided to quit their job. Particularly, youth and young adults also used similar approaches such as analyze and solve to prevent future stress occurrences on the job. By analyzing and attempting to solve a stressor at hand, participants can then understand how to approach and combat a stress-related challenge issue if it occurs again. For other participants in this study, breaking tasks into more manageable tasks helped alleviate feelings of stress and/or anxiety on the job. This finding is consistent with that of Hurlbutt and Chalmers who found people with ASD tend to break tasks into more controllable steps to help alleviate feelings of being overwhelmed [18].

In regard to emotion-focused coping approaches, one study found that university students with ASD coped by taking frequent extended breaks and changing their school disciplines towards their interests [247]. These results parallel the results in this study as some participants used emotion-focused coping (e.g., distractibility) to reduce negative emotions and feelings related to stress. Mindfulness and calming techniques have been reported in the literature among people with ASD as helping to improve emotion regulation in adolescents with ASD and can be customized to individual demands, symptoms and cognitive capability $[98,248]$. In this study, many participants reported using a variety of breathing techniques to reduce feelings of negativity or negative emotions on the job. Participants also described the significance of having social support in the workplace, both to ask for help during stressful situations and also to lean on during times of trouble (e.g., venting). These findings reflect the results in the literature as studies have shown that mentoring can be an effective type of support for people with ASD in an employment setting [22,249]. What is surprising is that 
social support was reported in both emotional and problem- focused coping strategies despite the various social challenges that participants face in the workplace. That is to say, even though social challenges occur in the workplace, people with ASD also rely on social support to relieve feelings of stress and/or anxiety on the job.

Similar to the findings of this study, adults without disabilities use both problem-focused coping and emotion-focused coping approaches in an employment setting in attempts to resolve a stressful situation in the workplace. For instance, Mantler et al. found that adults without disabilities who worked in the technology industry often resorted to problem-focused coping approaches to reduce stress on the job [250]. In addition, similar to the findings of this study related to social support, another study found that a majority of employees without disabilities that worked in a university resorted to coping strategies associated with seeking social support [251]. Other studies have also found using social support to be the most commonly used coping strategy among employees without disabilities [252-254]. These study findings suggest that coping strategies are utilized by people with and without disabilities and can be employed to combat feelings of stress and anxiety on the job. Although coping strategies are used by both people with and without disabilities, these findings help us to understand individualized coping mechanisms and how these strategies are not always universal. Instead, these coping strategies are context and situation dependent and can vary across time. These findings raise intriguing questions regarding the nature and extent of coping mechanisms and how they can differ based on the situation and thus warrant further research.

\subsubsection{Coping Strategies in Relation to the Transactional Model of Stress and Coping:}

The Transactional Model of Stress and Coping (TMSC) was selected for this study because of its characterizations of "problem-focused coping" and "emotion-focused coping" and the way this theory positions the coping process.

Overview of TMSC: TMSC explains coping as a phenomenon that entails cognitive and behavioural responses that people use in attempts to manage stressors [255]. This theory posits that coping strategies are vital in order to appraise a stressor. According to TMSC, people monitor and appraise stressful events differently in the workplace [9]. In addition, this 
model conceptualizes stress and managing stress as an individualistic process and not at all the same for everyone [157]. This theory is extremely important because this model favours looking into the characteristics of individuals and their own unique ways of coping given a stressful circumstance [256].

Many of the coping strategies aligned with the categories of emotion-focused coping and problem-focused coping as characterized with TMSC. In line with the theory, the findings from this study support the fact that coping is individual and context dependent. In addition, the findings from this study demonstrate the fact that coping does not necessarily have to be singular (i.e., only one style of coping). In fact, many of the participants reported using more than one style of coping in times of stress and/or anxiety in the workplace. This model helps individuals compartmentalize different strategies for various situations, allowing individuals to manage a stressful situation in a manner unique and beneficial to them. In addition, this theory gives insight on the context of the work environment and how influential the environment can be for an appraisal process, thereby stressing how different factors (e.g., type of job, environment, etc.) affect an individual's stress management process [257]. This theory relates to the findings from this study because participants described coping strategies that were tailored to their work environment. For instance, participants who used breathing exercises described that it was a convenient form of coping because it did not require much time or space. This finding parallels the TMSC framework because individuals are able to cope in a manner that works for them, while not interfering with their work task or environment. Similarly, the TMSC highlights flexibility in coping. In other words, it can show how stressors and the situation in which they occur can alter across time, which ultimately shows how the appraisal process can change. This model is not stagnant, in that it is everchanging, giving individuals opportunities to refine, develop and employ strategies that work for them in a certain context and time.

\subsubsection{Pushing Through in the Workplace}

Besides problem and emotion-focused coping approaches, many participants described pushing through as another coping style utilized in the workplace. For people with ASD, sticking to routine and insistence on sameness can be seen as a moderating factor for stress and in itself, can be seen as a coping strategy used to reduce stress [98]. However, pushing 
through is not necessarily a positive form of coping. Problem-focused coping incites a positive outlook towards a problem, which is a crucial intermediary between traits related to ASD and anxiety symptoms [258,259]. Unlike problem-focused coping, people with ASD may use pushing through to overwork themselves and as a result may ignore indicators that they need to take a break [247]. In the literature, other strategies define pushing through as suppression (e.g., suppressing emotional expression), which has been shown to increase negative emotions $[124,260,261]$. Therefore, pushing through can be seen as a maladaptive form of coping, which can negatively impact one's mental health. For many participants in this study, they reported to pushing through because they had no choice but to finish their tasks at work despite feeling stressed and/or anxious on the job. There is limited literature regarding pushing through and the negative effects it may have on people with ASD, especially in the context of employment. In addition, the results from this study demonstrate that the burden of coping and assimilating into the environment is often placed on the individual with ASD in the workplace. However, in the same vein, the findings demonstrate that retaining employment and successfully maintaining employment depends on a myriad of factors such as the environment and the policies around employment for people with ASD. Therefore, it is vital for employers and coworkers to be aware and understanding of the spectrum of ASD. It is important to further understand the impact of coping strategies, such as pushing through, because it may contribute to burnout and negative emotions on the job, which may impact job performance as seen through the results of this study.

\subsubsection{How Coping Helped Me}

Overall, participants often felt positive emotions after using emotion or problem-focused coping approaches in the workplace. Coping is important because an individual's perception of their ability to cope with a stressor is vital for the perception of distress [118]. Participants in this study echoed this sentiment especially when they faced reoccurring stress and/or anxiety inducers in the workplace. By already having established effective coping strategies, participants are able to combat stress and/or anxiety on the job. This study also emphasized the fact that coping is essential in the workplace because it helps improve mood, make tasks more manageable, and improve mental health. Further work is required to investigate and explore other styles of coping and their associated impact among youth and young adults with ASD. 


\subsection{Implications}

\subsubsection{Implications for Employers}

The implications from this study highlight the potential of using the experiences of youth and young adults with ASD to promote ASD awareness in the workplace and contribute to how current interventions related to employment are created and implemented. As demonstrated from the results from the study, there is a need for employers to promote a warm and inviting environment for youth and young adults with ASD in the workplace. In the same vein, a welcoming and inviting environment may have been a result of some participants having the opportunity to individualize their workplace environment, which may have led to a more positive experience at work. Hence, the results from this study underscore the fact that there are multiple factors at play in the workplace environment and that due to the heterogeneity of ASD, employment experiences can vary.

For some participants in this study, common challenges on the job related to social and communication difficulties in the workplace. The responsibility of adhering to social norms often falls on the individual with ASD. However, employers can help to alleviate this pressure by embracing individual characteristics of people with ASD and being more informed of the heterogeneity of features of ASD, especially in the context of employment. Since the features of ASD can vary from individual to individual, preferences and limitations can change based on the individual. Thus, it is important to recognize and acknowledge individual experiences and to not categorize youth and young adults with ASD in rigid classifications. Similarly, it is important for employers to consider the work skills and an individual's capacity to work rather than focusing on social skills or social norms in the workplace. Other strategies that employers can implement consist of more inclusive workplace practices: (1) training all employees in attempts to improve productivity, (2) partnering with disability organizations, and (3) ensuring leaders of a workplace to consistently show inclusive attitudes $[145,193,194]$. It is also evident from the study findings that diversity and inclusivity training can help employers and employees without ASD to be better informed of disabilities in the workplace. Increased knowledge and understanding of ASD on a managerial level (e.g., various communication strategies that are both verbal and written) can better facilitate employment success for people with ASD [116]. The findings from this study provide support that youth and young adults with ASD are capable of completing tasks but do so in a way that 
prevents stress and anxiety on the job. Hence, to foster an inclusive work environment, employers can allow workers to take initiative on executing tasks in a way that gives them comfort and limits personal stress.

The results from this study also highlight the need for employers to clearly communicate job descriptions instead of relying on generic descriptions. Rather than generic descriptions, roles should be devised to maximize the potential and skills of individuals with ASD [194,229]. Precise literal language to describe jobs can be helpful because applicants with ASD may interpret words more literally [194]. Many businesses and places of employment list similar characteristics for vacant positions (e.g., hardworking, team player, etc.). However, hiring and recruiting managers should critically think about each position and reflect to see what each role requires and describe the jobs accordingly. For instance, if the position calls for a cashier, explaining role-related characteristics can give people with ASD an idea of what the job will entail (e.g., exceptional social communication/interaction skills or required vs. preferred skills). It is important for employers to set clear expectations for tasks and working arrangements (e.g., tasks, work hours, time expected to complete tasks etc.) so that people with ASD are more informed and aware of the work environment and what is expected of them. The voices and concerns of the participants can further inform employers on how to be more inclusive in the workplace and potentially extend to better recruitment practices. For instance, Austin and Sonne call on adjustments to work conditions through novel approaches to hiring, training, and work design to further include talents and skills from people who are neurodiverse [262]. The concept of maintaining employment can be further assessed by possibly looking at a continued assessment of employee needs on an ongoing basis or change in role [194]. For employees that have multiple roles within a job (e.g., service sector roles), it is important for employers to have this continued assessment because a different role can lead to different job responsibilities. Findings from this study also suggest that when employers have experienced knowledge and a willingness to learn about ASDs in the workplace, employees with ASD have a far more positive relationship with their employers in the workplace.

The study findings revealed that participants felt stressed and anxious on the job, especially in the midst of COVID-19. These findings, while preliminary, suggest that employers offer vocational support (e.g., consideration of the employee with ASD's preferred 
working condition), especially during unprecedented events such as COVID-19. Due to the heterogeneity of ASD, it is important for employers to understand and acknowledge jobrelated limitations of youth and young adults with ASD in the workplace. Some individuals may be more comfortable working from home whereas others may wish to have a more balanced work schedule (e.g., working from home half the time). Under COVID-19 restrictions, if employers allow flexibility to have a balanced work-from-home and in-person working environment, it may help individuals with ASD succeed on the job. The literature shows that transitioning to working from home can perhaps maintain productivity [263]. In addition, job-related adjustments and supports, which have been necessary for the success of employees with ASD [216], should also continuously monitor overall wellbeing and happiness with the role itself.

The findings of this study also provide policy-related implications. For instance, employers can implement a job descriptions policy where employees are made aware of their duties and work-related skills required through job descriptions and performance expectations. The results from this study also highlight the need for inclusive workplace policies where organizations cultivate a friendly workplace environment and every employee is treated with respect, fairness and dignity, which includes maintaining an environment that is free of discrimination and harassment. Such policies can help foster a positive and inviting workplace environment.

\subsubsection{Implications for Coworkers and Colleagues}

Throughout the study, participants described their varying relationships with their coworkers and colleagues. Some participants recalled positive experiences with working with their coworkers and colleagues. From the findings, it was evident that coworkers and colleagues are an important source of social support for participants with ASD (e.g., peer mentoring). In the literature, it shows that peer support can help alleviate work stress and impact of task demands on the job [22]. However, negative experiences with coworkers and colleagues were also mentioned in the interviews, which contributed to feelings of stress and anxiety on the job. People with ASD may speak in a blunt manner and speak on topics that are familiar only to them [46]. As a coworker and colleague, it is important to be open, friendly, and warm to employees with ASD in the workplace, however communication styles can make 
this a challenge. People with ASD may have a preferred mode of communication with coworkers and colleagues, but coworkers and colleagues may be unaware of these preferences. In addition, preferred communication styles can change depending on the person with ASD. The double empathy gap may play a role in the workplace as both parties (i.e., employees with ASD and neurotypical coworkers and colleagues) may not be understanding of one another. Thus, as a coworker and colleague the role of adaptability is important as communication preferences can vary from individual to individual. Perhaps coworkers and colleagues can use explicit and simple communication in the workplace and adapt to communication styles based on the individual with ASD in the workplace.

From the results of this study, judgmental opinions and mannerisms negatively impacted workplace experiences for youth and young adults with ASD. Therefore, the findings from this study highlight the need for ASD and diversity awareness training to promote more inclusive practices in the workplace. The responses from participants from this study also underscore the need for coworkers and colleagues to build strong social relationships with youth and young adults with ASD. This result may be explained by the fact that unfriendly and negative interactions can make the workplace environment challenging for people with ASD, which can affect the ability to make meaningful relationships in the workplace. The findings from this study also show that it is important to encourage cooperative work and participation of workers with ASD in activities because that can improve relationships in the workplace [194]. Among neurotypical coworkers and colleagues, the results show that it is important to understand ASD-related behaviours in the workplace. Misunderstandings of ASD-related behaviours (e.g., difficulties understanding social cues) may contribute to continued stigma of employees with ASD [194]. A supportive and welcoming environment is often linked to better outcomes for people with ASD [194]. Hence, as the findings demonstrate, it is important for coworkers and colleagues to take part in diversity and ASD awareness training to be better informed of ASD in the workplace and to foster a positive work environment. However, to truly ensure success in the workplace for youth and young adults with ASD, neurotypical coworkers and colleagues need to recognize and acknowledge how they are communicating and helping in providing adjustments and accommodations. 


\subsubsection{Implications for People with ASD}

The findings from the study provide novel insight on the impact of COVID-19, especially the contrast between working in-person and working-from-home. Specifically in relation to COVID-19, participants mentioned negative experiences working with customers, overall stress associated with the pandemic, and inability to work-from-home. These findings have important implications for developing employment interventions (e.g., protocols and work mandates) to manage work environments during COVID-19. Youth and young adults with ASD in this study also underscored the significance of being able to foresee and predict stress occurrences on the job in order to better prepare and minimize feelings of stress and/or anxiety on the job. These findings raise intriguing questions regarding the nature and extent of stress preventive strategies in the workplace and their very use and impact in unforeseeable circumstances such as COVID-19.

The results from this study also show that people with ASD can benefit from preemployment programs and workshops. Similar to the findings of predicting workplace stressors, workshops that entail situating stressful scenarios and listing out coping mechanisms in a healthy and positive manner can potentially benefit youth and young adults who are employed or looking for work. Workshops that help with training to help with interpersonal skills such as social awareness, communication, teamwork, and networking can improve workplace communication among people with ASD [194]. As shown through the participants' accounts of social difficulties on the job, workshops to help improve communication in the workplace can potentially lead to positive workplace experiences and employee retention. In this study, some participants wanted to change their current work position to a position that tailored to their preferences and interests. From this study, youth and young adults with ASD may benefit from job intervention programs to job match interests and preferences with job roles, which may reduce feelings of stress and/or anxiety on the job. Focusing on strengths in the workplace can help individuals be matched with jobs that are suited to their skills and interests. Results from this study also have potential to inform youth and young adults with ASD who are currently looking for work of the advantages and disadvantages to disability disclosure in the workplace. From this study, participants mentioned both positive and negative experiences with disclosing their disability at work. Understanding and being more informed of the various experiences of disability disclosure in 
the context of employment can help youth and young adults with ASD who are looking for work because they can see the various outcomes to disclosing a disability in the workplace.

The findings from this study also demonstrate the powerful impact of stress in the workplace on youth and young adults with ASD. Although participants mentioned various coping strategies to combat feelings of stress and anxiety in the workplace, some coping mechanisms may negatively impact mental health (e.g., pushing through). For youth and young adults with ASD, it is important to find ways to positively cope in the workplace, instead of adding to the existing feelings of stress and anxiety on the job by using a negative coping strategy. Unique to this study, participants mentioned several coping strategies that can be used in a variety of workplace environments (e.g., grocery store, companies, working from home, etc.), which can help people with ASD who are cautious about utilizing coping strategies because of their work environment. As one study emphasized, there is a practical need to learn strategies for coping with stress due to psychosocial risks in the workplace, and by using coping strategies in the workplace, it can improve occupational health of employees [233]. This study demonstrates the role of coping in the workplace and how it can be used to minimize feelings of stress and anxiety on the job. For instance, having social supports on the job itself can motivate and minimize feelings of stress in youth and young adults with ASD. Support on the job improves overall functioning of workers with intellectual disabilities and also improves work outcomes and overall quality of life [233]. In this study alone, participants described an overwhelming number of positive experiences with coping and an overall positive impact of coping in the workplace. These results have the potential to aid job seekers with ASD or people with ASD who are currently employed but have not yet developed coping strategies to understand and be informed of the coping strategies that can be used in the workplace.

\subsubsection{Implications for Rehabilitation}

The findings from this study suggest that it may be beneficial for clinicians to provide psychological help and resources for employment-related challenges for youth and young adults with ASD in competitive employment. As the results show, clinicians can offer resources (e.g., individualized support plans) to help cope with stress and anxiety in the workplace. Individual support plans and resources can benefit youth and young adults with 
ASD as it can be tailored to the individual and their specific needs. Clinicians are involved in the diagnosis for people with ASD and for people that are diagnosed later in life (i.e., during or after high school), it is important for them to implement transition-related services, education and resources for stress and anxiety [194]. The results from this study can help inform clinicians of the stress and anxiety inducers in the workplace among people with ASD, which can manifest as social isolation, difficulty concentrating, and increased relational challenges $[31,113,240]$. Since many of these symptoms are related to the symptomology of ASD, they may be evaluated by clinicians as part of the ASD diagnosis, which means the feelings of stress and/or anxiety under these core symptoms can be left unnoticed and untreated [264]. Hence, it is important for clinicians to keep this in mind when performing assessments and contributing to the treatment planning.

The findings from this study have clear implications for transition services and employment-programs, suggesting that social and communication skill-building should be emphasized in order to help improve employment outcomes among youth and young adults with ASD. Similarly, the evidence from this study indicates that employment programs should focus on skillset, interests, and needs of individuals with ASD when providing job matching services [62]. Clinicians should also be encouraged to collaborate with transition services and employment programs to initiate work-related training and social skills training before a youth finishes high school [265]. The findings from this study also suggest that service providers and clinicians should also support people with ASD to advocate for their workplace requests, which includes disclosing a disability in the workplace and asking for workplace adjustments and accommodations [33]. By doing so, people with ASD can be aware of what accommodations they need in the workplace and feel supported in the process. Similarly, employment-specialists and/or job coaches can provide neurotypical employers and coworkers with the skills training to communicate more effectively with colleagues with ASD.

There is a paucity of literature on stress and trauma-informed treatment strategies for people with ASD, which often leaves clinicians who work with people with ASD dealing with past trauma-related experiences without guidance [113]. Thus, the findings of this study related to past experiences have potential to help clinicians and service providers on a basic 
level of providing support and resources for people with ASD who are working in an employment setting.

\subsubsection{Study Strengths and Limitations}

This study had several strengths. A key strength of the present study was the first-hand, experiences with coping in relation to stress and anxiety among youth and young adults with ASD. This is the one of the only studies that explores coping in relation to work-related stress and anxiety, especially in this population. Another strength to this study included the ability to utilize online research methods (i.e., Zoom Communications) for data collection among individuals in this population, which allowed for recruitment across all of Canada. Another key strength was the novel work-related experiences that were discussed during the interviews as I was able to collect data during the first wave of COVID-19. A final strength to this study included the ability to understand more in-depth the workplace environment among youth and young adults with ASD and how elements in a workplace environment (i.e., disclosing a disability) can truly impact an individual's working experience.

The findings from this study should be understood with the following limitations in mind. Firstly, participants self-reported their ASD diagnosis prior to partaking in this study. Hence, formal diagnoses of participants were not confirmed. The study relied on self-reported ASD diagnosis as a result of the lack of access to the participant's medical documentation and records. Taken together, it was possible for this study to include participants that did not actually have ASD. A second limitation for this study was that this sample consisted of youth and young adults from Canada only. Youth and young adults with ASD living in other countries besides Canada may have different workplace experiences and practices due to different policies, cultures, and languages. Hence, the experiences from the participants in this study cannot be transferrable to the entire ASD population. The third limitation was that the chat function was turned off on Zoom, which may have impacted participants' responses to this study. Some participants may be shy or more comfortable with typing/writing their answers to the interview questions. However, due to the possibility of information being saved/leaked from the chat function, the chat function was turned off and not utilized in the data analysis process, which may have limited some participants' ability to reflect and voice their thoughts. A fourth limitation of this study could be due to using the TMSC theory to 
frame the different styles of coping. This may be considered a limitation because an overreliance on theory (i.e., definitions and concepts) can essentially prevent the salience and significance of data-driven information from pulling through [266]. In the same vein, using a hybrid approach for analysis can be seen as a limitation as a priori template codes can limit characterizations of coping into problem- or emotion-focused coping as opposed to participants describing and making sense of what coping means to them. Since coping was characterized through problem-focused or emotion-focused, it may have limited participant interpretations of what coping means and how they are differentiated. Put differently, by focusing on coping through a priori template codes, it may have led to a dichotomous categorization (i.e., not allowing fluidity in the types of coping employed). Thus, without a priori template codes, there may have been different interpretations of coping mechanisms. In contrast, by utilizing a priori template codes, it helped to categorize experiences while still allowing for unique experiences to be highlighted throughout the analysis process. Since we looked into the ways youth and young adults with ASD cope, this hybrid approach also helped to further understand the similarities and contrasts between the information in the dataset. In addition, the hybrid approach allowed for other forms of coping to emerge from the dataset (e.g., pushing through) through inductive coding. Thus, despite the risks, this analytic approach does not impose risk of an over-focus of data in light of a stress and/or coping orientation. Overall, these limitations highlight areas for investigation in further research studies.

\subsubsection{Future Directions}

There are a number of potential directions for future studies, which will build on the current research findings. One important finding to this study was the types of stress and/or anxiety inducers that participants experienced at their place of employment. Further assessing the impact of coping to various stress inducers can help enhance our understanding of the importance of coping strategies in the workplace and assess its effectiveness on mental health. For instance, how does the role of social support impact the levels of stress on the job? Perhaps further studies can also look at the role of coping before and after stress and anxietyinducing situations to see its role in the workplace. Since there was no specific focus on employers in this study (i.e., no employers in this study sample), further research can look at the role of employers and colleagues and their perceptions of stress and anxiety inducing 
situations in the workplace. Since this study focused on employee perspectives on stress and anxiety in the workplace, future studies can further look at employer and coworker perceptions of the impact of stress in the workplace for people with ASD. Specifically, studies entailing focus groups with employers, coworkers, and employees with ASD can be beneficial to understand the impact of stress and anxiety on people with ASD in the workplace. Understanding and learning the impact of stress and anxiety in the workplace can help employers and coworkers be better aware and informed of their own role and how their thoughts and actions can contribute to stress and anxiety in the workplace.

The findings from this study also highlight the need for employers and coworkers to participate in diversity and inclusion training in the workplace to help facilitate a positive work environment for people with ASD. In the same manner, it may be vital for employmentspecialists or job coaches to provide neurotypical employers and coworkers with skills training to better communicate with employees with ASD. Future research studies can further uncover the relationship between these trainings and the overall feelings of stress and anxiety for people with ASD in the workplace. Specifically, studies can uncover the impact and overall effectiveness of these trainings for people with ASD in the workplace. Since a few participants described negative relationships with their coworkers and/or supervisor, it is important to examine the role of awareness and knowledge of ASD, especially for neurotypical employers and coworkers. Given the limited literature available on coping among youth and young adults with ASD, this study addressed this gap in the literature by uncovering coping mechanisms in the context of employment among youth and young adults with ASD. There is abundant room for further progress in determining the role of coping in employment among people with ASD. To develop a full picture of the value of coping in the workplace among youth and young adults with ASD, exploring why and how people with ASD approach and utilize certain strategies can help: (1) identify the types of support that people with ASD need in the workplace, (2) employers be better informed of how they can foster a positive work environment, and (3) understand the quality of coping (e.g., impact on mental health) employed in the workplace. 


\section{Chapter 6}

\section{Conclusion}

For typical young adults, obtaining employment is one of the signs of successful transition into adulthood [267]. Unfortunately, securing and retaining employment is an ongoing challenge for people with ASD, which can lead to individuals with ASD experiencing higher levels of stress on the job [21]. Increased on the job work demands can lead to increased levels of stress and anxiety for employees with ASD [120,235].

Furthermore, a prominent barrier to gaining and sustaining employment among youth and young adults with ASD includes coping with the social demands in the workplace [28]. Social challenges in the workplace can also induce feelings of stress and anxiety on the job [29,30]. Despite the extensive literature on employment among youth and young adults with ASD, there seems to be a paucity of information on how individuals with ASD maintain employment, especially when faced with stress and anxiety on the job. Among youth and young adults with ASD, stress and anxiety on the job can be reasons as to why employment is not successfully attained. Therefore, it is important to uncover the ways in which youth and young adults cope with the numerous stress and anxiety inducing situations that can occur in an employment setting.

The purpose of this current study was to understand the subjective feelings of stress and anxiety that youth and young adults with ASD experience in competitive employment. This qualitative study explored the experiences of youth and young adults with ASD as they faced stress and anxiety inducing situations in the workplace, from the perspective of these youth and young adults themselves. The second aim of this study was to understand the subjective coping strategies used by youth and young adults with ASD when faced with work-related stress or anxiety. This study has shown several stress and anxiety inducers in the workplace, which negatively impacted participant's experience in the workplace. Youth and young adults with ASD in this study also described the various coping mechanisms they utilized to combat these stress and anxiety inducers in the workplace. The findings have provided a deeper insight into the mechanisms of coping and how coping is individual and context and situation dependent, which can vary across time. In this study, youth and young adults with ASD 
described personal positive and negative aspects of the workplace, which impacted their employment experiences. Throughout these experiences, participants often expressed how crucial ASD knowledge and awareness are in informing people in the workplace (i.e., coworkers and employers) about potential employment-related challenges (e.g., difficulties with social communication). Interestingly, several salient workplace factors seemed in opposition (e.g., work environment, disability disclosure, and asking for adjustments), however, these subthemes were rather interrelated. The interrelation between these subthemes truly highlight individual experiences to employment and how each experience (i.e., both positive and negative) can vary depending on the individual. Furthermore, this study contributes to our understanding of ASD and disability awareness in the workplace and the large roles that employers and coworkers play in the success of youth and young adults with ASD in competitive employment.

Further studies regarding the role of stress and anxiety in the workplace are needed to better understand their associated impact on employment retention. Overall, this study provides evidence for the need to continue to explore the various styles of coping in the workplace among youth and young adults with ASD. Further research needs to examine more closely the links between stress and anxiety and coping in the workplace to understand their impact on employment maintenance among youth and young adults with ASD. 


\section{References}

1. Holwerda A, van der Klink JJL, Groothoff JW, et al. Predictors for Work Participation in Individuals with an Autism Spectrum Disorder: A Systematic Review. Journal of occupational rehabilitation. 2012;22(3):333-352.

2. LaRue RH, Maraventano JC, Budge JL, et al. Matching Vocational Aptitude and Employment Choice for Adolescents and Adults with ASD. Behavior Analysis in Practice. 2019 2019/12/19.

3. Solomon C. Autism and Employment: Implications for Employers and Adults with ASD. Journal of autism and developmental disorders. 2020.

4. American Psychological Association. Anxiety 2020 [April 1, 2020]. Available from: https://www.apa.org/topics/anxiety/

5. American Psychiatric Association. Diagnostic and statistical manual of mental disorders. 5th ed. Arlington, VA: American Psychiatric Association; 2013.

6. Health NIoM. Autism Spectrum Disorder. 2018.

7. Taylor JL, Seltzer MM. Employment and Post-Secondary Educational Activities for Young Adults with Autism Spectrum Disorders During the Transition to Adulthood. Journal of Autism and Developmental Disorders. 2011;41(5):566-574.

8. Parent W, Unger D, Gibson K, et al. The role of job coach: orchestrating community and workplace supports. American rehabilitation. 1994;20(3):2.

9. $\quad$ Lazarus RS, Folkman S. Stress, appraisal, and coping. 1984. English.

10. Robertson K, Frydenberg E. Coping strategies used by young people with autism spectrum disorders. Children Australia. 2011;36(3):136-143.

11. First JM, Cheak-Zamora NC, Teti M, et al. Youth perceptions of stress and coping when transitioning to adulthood with autism: A photovoice study. Qualitative social work : QSW : research and practice. 2019;18(4):601-620.

12. Bedrossian L. Understand autism meltdowns and share strategies to minimize, manage occurrences. Disability Compliance for Higher Education. 2015;20(7):6-6.

13. Galarneau D, René Morissette and Jeannine Usalcas. What has changed for young people in Canada? Insights on Canadian Society. July. Statistics Canada Catalogue; 2013.

14. National Research C, editor Background paper: pathways to young adulthood and preventive interventions targeting young adults2013: National Academies Press (US).

15. Arnett J. Emerging adulthood: A theory of development from the late teens through the twenties. American Psychologist.55(5):469-480.

16. Sawyer SM, Azzopardi PS, Wickremarathne D, et al. The age of adolescence. Lancet Child Adolesc Health. 2018;2(3):223-228.

17. Levy SE, Mandell DS, Schultz RT. Autism. The Lancet (British edition). 2009;374(9701):1627-1638.

18. Hurlbutt K, Chalmers L. Employment and adults with Asperger syndrome. Focus on autism and other developmental disabilities. 2004;19(4):215-222.

19. Smith Frank A, Lugas J. Vocational Rehabilitation Employment Outcomes for Transition-Age Youth with Autism and Other Disabilities. Data Note, Number 26. Institute for Community Inclusion. 2010.

20. Roux A, Shattuck P, Rast J, et al. National Autism Indicators Report: Transition into young adulthood. 2015. 
21. Baldwin S, Costley D, Warren A. Employment Activities and Experiences of Adults with High-Functioning Autism and Asperger's Disorder. Journal of Autism and Developmental Disorders. 2014;44(10):2440-2449.

22. Nicholas DB, Mitchell W, Dudley C, et al. An Ecosystem Approach to Employment and Autism Spectrum Disorder. Journal of autism and developmental disorders. 2018;48(1):264-275.

23. Scott M, Jacob A, Hendrie D, et al. Employers' perception of the costs and the benefits of hiring individuals with autism spectrum disorder in open employment in Australia. PLoS ONE. 2017;12(5):e0177607.

24. Kéita L, Guy J, Berthiaume C, et al. An early origin for detailed perception in Autism Spectrum Disorder: Biased sensitivity for high-spatial frequency information. Scientific reports. 2014;4(1):5475-5475.

25. Jiang YV, Palm BE, DeBolt MC, et al. High-precision visual long-term memory in children with high-functioning autism. Journal of Abnormal Psychology. 2015;124(2):447--456.

26. Lorenz T, Frischling C, Cuadros R, et al. Autism and Overcoming Job Barriers: Comparing Job-Related Barriers and Possible Solutions in and outside of AutismSpecific Employment. PloS one. 2016;11(1):e0147040.

27. Gerhardt PF, Cicero F, Mayville E. Employment and Related Services for Adults with Autism Spectrum Disorders. In: Volkmar FR, Reichow B, McPartland JC, editors. Adolescents and Adults with Autism Spectrum Disorders. New York, NY: Springer New York; 2014. p. 105-119.

28. Muller E, Schuler A, Burton BA, et al. Meeting the vocational support needs of individuals with Asperger Syndrome and other autism spectrum disabilities. Journal of Vocational Rehabilitation. 2003;18(3):163--175.

29. Hayward SM, McVilly KR, Stokes MA. Autism and employment: What works. Research in autism spectrum disorders. 2019;60:48-58.

30. Goldfarb Y, Gal E, Golan O. A conflict of interests: A motivational perspective on special interests and employment success of adults with ASD [Developmental Disorders \& Autism 3250]. Journal of Autism and Developmental Disorders. 2019;49(9):39153923.

31. Bishop $\square$ Fitzpatrick L, Mazefsky CA, Minshew NJ, et al. The Relationship Between Stress and Social Functioning in Adults With Autism Spectrum Disorder and Without Intellectual Disability. Autism Research. 2015;8(2):164-173.

32. Anderson C, Butt C, Sarsony C. Young Adults on the Autism Spectrum and Early Employment-Related Experiences: Aspirations and Obstacles. Journal of autism and developmental disorders. 2020.

33. Lindsay S, Osten V, Rezai M, et al. Disclosure and workplace accommodations for people with autism: a systematic review. Disability and Rehabilitation. 2019:1--14.

34. Norstedt M. Work and Invisible Disabilities: Practices, Experiences and Understandings of (Non)Disclosure. Scandinavian journal of disability research : SJDR. 2019;21(1):1424.

35. Allen S, Carlson G. To conceal or disclose a disabling condition? A dilemma of employment transition. Journal of Vocational Rehabilitation. 2003;19(1):19-30.

36. Georgiou C, Espahbodi S, De Souza L. Preparing for the world of work: An exploratory study of disabled students' experiences of work placement. Journal of Education and Work. 2011 02/27;25:1-14. 
37. Gal E, Selanikyo E, Erez AB-H, et al. Integration in the vocational world:How does it affect quality of life and subjective well-being of young adults with ASD. International Journal of Environmental Research and Public Health. 2015;12(9):10820-10832.

38. Hendricks DR, Wehman P. Transition From School to Adulthood for Youth With Autism Spectrum Disorders: Review and Recommendations. Focus on autism and other developmental disabilities. 2009;24(2):77-88.

39. Oswald TM, Winder-Patel B, Ruder S, et al. A Pilot Randomized Controlled Trial of the ACCESS Program: A Group Intervention to Improve Social, Adaptive Functioning, Stress Coping, and Self-Determination Outcomes in Young Adults with Autism Spectrum Disorder. Journal of autism and developmental disorders. 2018;48(5):17421760.

40. Lai M-C, Baron-Cohen S. Identifying the lost generation of adults with autism spectrum conditions. The Lancet Psychiatry. 2015;2(11):1013-1027.

41. Seymour M, Wood C, Giallo R, et al. Fatigue, Stress and Coping in Mothers of Children with an Autism Spectrum Disorder. Journal of autism and developmental disorders. 2012;43(7):1547-1554.

42. Shattuck PT, Narendorf SC, Cooper B, et al. Postsecondary Education and Employment Among Youth With an Autism Spectrum Disorder. Pediatrics (Evanston). 2012;129(6):1042-1049.

43. Taylor JL, Henninger NA, Mailick MR. Longitudinal patterns of employment and postsecondary education for adults with autism and average-range IQ. Autism : the international journal of research and practice. 2015;19(7):785-793.

44. Ohl A, Grice Sheff M, Small S, et al. Predictors of employment status among adults with Autism Spectrum Disorder. (1875-9270 (Electronic)).

45. Lindsay S, Cagliostro E, Leck J, et al. Employers' perspectives of including young people with disabilities in the workforce, disability disclosure and providing accommodations. Journal of vocational rehabilitation. 2019;50(2):141-156.

46. Hedley D, Cai R, Uljarevic M, et al. Transition to work: Perspectives from the autism spectrum. Autism : the international journal of research and practice. 2018;22(5):528541.

47. Wehman P, Schall CM, McDonough J, et al. Effects of an employer-based intervention on employment outcomes for youth with significant support needs due to autism. Autism. 2017;21(3):276-290.

48. American Psychological Association. Diagnostic and statistical manual of mental disorders. Fourth ed.: AMER PSYCHOLOGICAL ASSOC; 1994.

49. Ben-Sasson A, Hen L, Fluss R, et al. A Meta-Analysis of Sensory Modulation Symptoms in Individuals with Autism Spectrum Disorders. Journal of Autism and Developmental Disorders. 2009 2009/01/01;39(1):1-11.

50. Data \& Statistics on Autism Spectrum Disorder. Centers for Disease Control and Prevention; 2018.

51. Loomes RD, Hull LM, Mandy WPLDP. What is the Male-to-Female Ratio in Autism Spectrum Disorder? A Systematic Review and Meta-Analysis. Journal of the American Academy of Child and Adolescent Psychiatry. 2017;56(6):466-474.

52. Lai M-C, Szatmari P. Sex and gender impacts on the behavioural presentation and recognition of autism. Current Opinion in Psychiatry. 2020;33(2).

53. Dworzynski KPD, Ronald APD, Bolton PPD, et al. How Different Are Girls and Boys Above and Below the Diagnostic Threshold for Autism Spectrum Disorders? Journal of the American Academy of Child and Adolescent Psychiatry. 2012;51(8):788-797. 
54. Duvekot J, van der Ende J, Verhulst FC, et al. Factors influencing the probability of a diagnosis of autism spectrum disorder in girls versus boys. Autism : the international journal of research and practice. 2017;21(6):646-658.

55. Lundström S, Mårland C, Kuja-Halkola R, et al. Assessing autism in females: The importance of a sex-specific comparison. Psychiatry research. 2019;282:112566-112566.

56. Canada DC. People diagnosed with autism are dealing with high unemployment rates 2016. Available from: https://disabilitycreditcanada.com/autism-high-unemploymentrate/

57. Stoddart K, Burke L, Muskat B, et al. Diversity in Ontario's Youth and Adults with Autism Spectrum Disorders: Complex Needs in Unprepared Systems. 2013.

58. Canada Go. Canadian Community Health Survey: Annual Component, 2017. 2017.

59. Zwicker J, Zaresani A, Emery JCH. Describing heterogeneity of unmet needs among adults with a developmental disability: An examination of the 2012 Canadian Survey on Disability. Research in developmental disabilities. 2017;65:1-11.

60. Austin RD, Pisano GP. Neurodiversity as a Competitive Advantage: Harvard Business Review . 2017.

61. Hagner D, Cooney BF. "I Do That for Everybody": Supervising Employees With Autism. Focus on autism and other developmental disabilities. 2005;20(2):91-97.

62. Hendricks D. Employment and adults with autism spectrum disorders: Challenges and strategies for success. Journal of Vocational Rehabilitation. 2010;32(2):125--134.

63. Cederlund M, Hagberg B, Billstedt E, et al. Asperger Syndrome and Autism: A Comparative Longitudinal Follow-Up Study More than 5 Years after Original Diagnosis. Journal of autism and developmental disorders. 2007;38(1):72-85.

64. Jennes-Coussens M, Magill-Evans J, Koning C. The quality of life of young men with Asperger syndrome: A brief report. Autism. 2006;10(4):403-414.

65. Hernandez B, McDonald K. Exploring the Costs and Benefits of Workers with Disabilities. Journal of Rehabilitation. 2010;76(3).

66. Cimera RE. The monetary benefits and costs of hiring supported employees: A pilot study. Journal of Vocational Rehabilitation. 2009;30(2):111-119.

67. Cimera RE, Cowan RJ. The costs of services and employment outcomes achieved by adults with autism in the US. Autism. 2009;13(3):285-302.

68. Roehrich HC, Grabanski J, Fischer D. Supporting adults with Asperger syndrome in the workforce: Implications for higher education practices. Marketing Management Journal. $2016 ; 1$.

69. Scott M, Falkmer M, Girdler S, et al. Viewpoints on Factors for Successful Employment for Adults with Autism Spectrum Disorder. (1932-6203 (Electronic)).

70. Gentry T, Lau S, Molinelli A, et al. The Apple iPod Touch as a vocational support aid for adults with autism: Three case studies. Journal of Vocational Rehabilitation. 2012;37:7585.

71. Gilson CB, Carter EW. Promoting social interactions and job independence for college students with autism or intellectual disability: A pilot study. Journal of autism and developmental disorders. 2016;46(11):3583-3596.

72. Hillier A, Campbell H, Mastriani K, et al. Two-year evaluation of a vocational support program for adults on the autism spectrum. Career Development for Exceptional Individuals. 2007;30(1):35-47.

73. McKnight-Lizotte M. Work-Related Communication Barriers for Individuals with Autism: A Pilot Qualitative Study. Australian Journal of Rehabilitation Counselling. 2018;24(1):12--26. 
74. Black MH, Mahdi S, Milbourn B, et al. Multi $\square$ informant International Perspectives on the Facilitators and Barriers to Employment for Autistic Adults. Autism research. 2020;13(7):1195-1214.

75. Shtayermman O. Peer Victimization in Adolescents and Young Adults Diagnosed with Asperger's Syndrome: A Link to Depressive Symptomatology, Anxiety Symptomatology and Suicidal Ideation. Issues in comprehensive pediatric nursing. 2007;30(3):87-107.

76. Burt DB, Fuller SP, Lewis KR. Brief report: competitive employment of adults with autism. Journal of autism and developmental disorders. 1991;21(2):237-42.

77. Scott M, Falkmer M, Falkmer T, et al. Evaluating the Effectiveness of an AutismSpecific Workplace Tool for Employers: A Randomised Controlled Trial. Journal of Autism and Developmental Disorders. 2018 2018/10/01;48(10):3377-3392.

78. Vogeley K, Kirchner JC, Gawronski A, et al. Toward the development of a supported employment program for individuals with high-functioning autism in Germany. European Archives of Psychiatry and Clinical Neuroscience. 2013 2013/11/01;263(2):197-203.

79. Patton E. Autism, attributions and accommodations. Personnel review. 2019;48(4):915934.

80. Shattuck PT, Seltzer MM, Greenberg JS, et al. Change in Autism Symptoms and Maladaptive Behaviors in Adolescents and Adults with an Autism Spectrum Disorder. Journal of autism and developmental disorders. 2006;37(9):1735-1747.

81. Smith MD. Autism and Life in the Community: Successful Interventions for Behavioral Challenges. P.H. Brookes Publishing Company; 1990.

82. Smith MD, Coleman D. Managing the behavior of adults with autism in the job setting. Journal of Autism and Developmental Disorders. 1986;16(2):145-154.

83. Myers SM, Johnson CP. Management of Children With Autism Spectrum Disorders. Pediatrics. 2007;120(5):1162.

84. Simpson RL, de Boer-Ott SR, Griswold DE, et al. 8: Adams, LG (2005). Autism spectrum disorders: Interventions and treatments for children and youth. Thousand Oaks, CA: Corwin Press.

85. Krieger B, Kinébanian A, Prodinger B, et al. Becoming a member of the work force: Perceptions of adults with Asperger Syndrome. Work. 2012;43(2):141-157.

86. Khalifa G, Sharif Z, Sultan M, et al. Workplace accommodations for adults with autism spectrum disorder: a scoping review. Disability and rehabilitation. 2019:1-16.

87. Bublitz DJ, Fitzgerald K, Alarcon M, et al. Verbal behaviors during employment interviews of college students with and without ASD. Journal of vocational rehabilitation. 2017;47(1):79-92.

88. Johnson TD, Joshi A. Dark clouds or silver linings? A stigma threat perspective on the implications of an autism diagnosis for workplace well-being. Journal of applied psychology. 2016;101(3):430-449.

89. Romualdez AM, Heasman B, Walker Z, et al. "People Might Understand Me Better": Diagnostic Disclosure Experiences of Autistic Individuals in the Workplace. Autism in Adulthood. 2021.

90. Sarrett J. Interviews, Disclosures, and Misperceptions: Autistic Adults' Perspectives on Employment Related Challenges. Disability Studies Quarterly. 2017 06/01;37.

91. Ju S, Roberts E, Zhang D. Employer attitudes toward workers with disabilities: A review of research in the past decade. Journal of Vocational Rehabilitation. 2013 01/01;38:113123.

92. Morgan RL, Alexander M. The employer's perception: Employment of individuals with developmental disabilities. Journal of vocational rehabilitation. 2005;23(1):39-49. 
93. Houtenville A, Kalargyrou V. People with Disabilities: Employers' Perspectives on Recruitment Practices, Strategies, and Challenges in Leisure and Hospitality. Cornell Hospitality Quarterly. 2012;53(1):40-52.

94. Morgan R, Schultz J. Towards an Ecological, Multi-Modal Approach to Increase Employment for Young Adults with Autism Spectrum Disorder. Journal of Applied Rehabilitation Counseling. 2012 03/01;43:27-35.

95. Cimera RE, Burgess S. Do adults with autism benefit monetarily from working in their communities? Journal of Vocational Rehabilitation. 2011;34:173-180.

96. Irvine A, Lupart J. Into the Workforce: Employers' Perspectives of Inclusion. Developmental Disabilities Bulletin. 2008 01/01;36.

97. Nicholas D, Mitchell W, Zulla R, et al. Perspectives of employers about hiring individuals with autism spectrum disorder: Evaluating a cohort of employers engaged in a job-readiness initiative. Journal of Vocational Rehabilitation. 2019 04/12;50:1-13.

98. Smith RS, Sharp J. Fascination and Isolation: A Grounded Theory Exploration of Unusual Sensory Experiences in Adults with Asperger Syndrome. Journal of Autism and Developmental Disorders. 2013 2013/04/01;43(4):891-910.

99. Scott M, Milbourn B, Falkmer M, et al. Factors impacting employment for people with autism spectrum disorder: A scoping review. Autism : the international journal of research and practice. 2019;23(4):869-901.

100. Nah Y-H, Brewer N, Young RL, et al. Brief Report: Screening Adults with Autism Spectrum Disorder for Anxiety and Depression. Journal of autism and developmental disorders. 2018;48(5):1841-1846.

101. Corbett BA, Muscatello RA, Blain SD. Impact of sensory sensitivity on physiological stress response and novel peer interaction in children with and without autism spectrum disorder. Frontiers in Neuroscience. 2016;10:278.

102. Hirvikoski T, Blomqvist M. High self-perceived stress and poor coping in intellectually able adults with autism spectrum disorder. Autism. 2015;19(6):752-757.

103. Cheak-Zamora NC, Teti M, Maurer-Batjer A. Capturing Experiences of Youth With ASD via Photo Exploration: Challenges and Resources Becoming an Adult. Journal of adolescent research. 2018;33(1):117-145.

104. Bellini S. The Development of Social Anxiety in Adolescents With Autism Spectrum Disorders. Focus on autism and other developmental disabilities. 2016;21(3):138-145.

105. Kanner L. Autistic disturbances of affective contact. Acta paedopsychiatrica. 1968;35(4):100-136.

106. Johnco C, Storch EA. Anxiety in youth with autism spectrum disorders: implications for treatment. Expert review of neurotherapeutics. 2015;15(11):1343-1352.

107. Kerns CM, Kendall PC, Zickgraf H, et al. Not to be overshadowed or overlooked: Functional impairments associated with comorbid anxiety disorders in youth with ASD. Behavior therapy. 2015;46(1):29-39.

108. Rodgers J, Glod M, Connolly B, et al. The relationship between anxiety and repetitive behaviours in autism spectrum disorder. Journal of autism and developmental disorders. 2012;42(11):2404-2409.

109. Wood JJ, Gadow KD. Exploring the nature and function of anxiety in youth with autism spectrum disorders. Clinical Psychology: Science and Practice. 2010;17(4):281.

110. Lai MC, Kassee C, Besney R, et al. Prevalence of co-occurring mental health diagnoses in the autism population: a systematic review and meta-analysis. The Lancet Psychiatry. 2019;6(10):819-829. 
111. van Steensel FJA, Heeman EJ. Anxiety Levels in Children with Autism Spectrum Disorder: A Meta-Analysis. Journal of Child and Family Studies. 2017 2017/07/01;26(7):1753-1767.

112. Gillott A, Standen PJ. Levels of anxiety and sources of stress in adults with autism. Journal of Intellectual Disabilities. 2007;11(4):359-370.

113. Fuld S. Autism Spectrum Disorder: The Impact of Stressful and Traumatic Life Events and Implications for Clinical Practice. (0091-1674 (Print)).

114. Simm W, Ferrario MA, Gradinar A, et al., editors. Anxiety and autism: Towards personalized digital health2016.

115. Camarena PM, Sarigiani PA. Postsecondary Educational Aspirations of HighFunctioning Adolescents With Autism Spectrum Disorders and Their Parents. Focus on Autism and Other Developmental Disabilities. 2009 2009/06/01;24(2):115-128.

116. Dreaver J, Thompson C, Girdler S, et al. Success Factors Enabling Employment for Adults on the Autism Spectrum from Employers' Perspective. Journal of autism and developmental disorders. 2020;50(5):1657-1667.

117. Pfeiffer B, Brusilovskiy E, Davidson A, et al. Impact of person-environment fit on job satisfaction for working adults with autism spectrum disorders. Journal of Vocational Rehabilitation. 2018 02/26;48:49-57.

118. Karasek R, Theorell T. Healthy work : stress, productivity, and the reconstruction of working life. New York: Basic Books; 1990.

119. Chen JL, Leader G, Sung C, et al. Trends in Employment for Individuals with Autism Spectrum Disorder: a Review of the Research Literature. Review Journal of Autism and Developmental Disorders. 2015 2015/06/01;2(2):115-127.

120. Parr AD, Hunter ST, Ligon GS. Questioning universal applicability of transformational leadership: Examining employees with autism spectrum disorder. The Leadership quarterly. 2013;24(4):608-622.

121. Skinner EA, Edge K, Altman J, et al. Searching for the Structure of Coping: A Review and Critique of Category Systems for Classifying Ways of Coping. Psychological Bulletin. 2003;129(2):216-269.

122. Erica F, Ramon L. Teaching Coping to Adolescents: When and to Whom? American educational research journal. 2000;37(3):727-745.

123. Frith U, Mira M. Autism and Asperger syndrome. Focus on Autistic Behavior. 1992;7(3):13-15.

124. Gross JJ, John OP. Individual differences in two emotion regulation processes: implications for affect, relationships, and well-being. (0022-3514 (Print)).

125. Mazefsky CA, Herrington J, Siegel M, et al. The Role of Emotion Regulation in Autism Spectrum Disorder. Journal of the American Academy of Child \& Adolescent Psychiatry. 2013;52(7):679-688.

126. Konstantareas MM, Stewart K. Affect Regulation and Temperament in Children with Autism Spectrum Disorder. Journal of Autism and Developmental Disorders. 2006 2006/02/01;36(2):143-154.

127. Samson AC, Phillips JM, Parker KJ, et al. Emotion dysregulation and the core features of autism spectrum disorder. Journal of Autism and developmental Disorders. 2014;44(7):1766-1772.

128. Mazefsky CA. Emotion Regulation and Emotional Distress in Autism Spectrum Disorder: Foundations and Considerations for Future Research. Journal of Autism and Developmental Disorders. 2015 2015/11/01;45(11):3405-3408. 
129. Mazefsky CA, Borue X Fau - Day TN, Day Tn Fau - Minshew NJ, et al. Emotion regulation patterns in adolescents with high-functioning autism spectrum disorder: comparison to typically developing adolescents and association with psychiatric symptoms. Autism Research. 2014;7(3):344-354.

130. Sáez-Suanes GP, García-Villamisar D, Armentia A, et al. Emotion regulation as a mediator between depressive symptoms and autism spectrum disorder (ASD) in adults with ASD and intellectual disabilities. Research in autism spectrum disorders. 2020;78:101654.

131. Dachez J, Ndobo A. Coping Strategies of Adults with High-Functioning Autism: A Qualitative Analysis. Journal of Adult Development. 2018;25(2):86-95.

132. Pouw LBC, Rieffe C, Stockmann L, et al. The link between emotion regulation, social functioning, and depression in boys with ASD. Research in autism spectrum disorders. 2013;7(4):549-556.

133. Milner V, McIntosh H, Colvert E, et al. A Qualitative Exploration of the Female Experience of Autism Spectrum Disorder (ASD). Journal of Autism and Developmental Disorders. 2019 2019/06/01;49(6):2389-2402.

134. Bargiela S, Steward R, Mandy W. The Experiences of Late-diagnosed Women with Autism Spectrum Conditions: An Investigation of the Female Autism Phenotype. Journal of Autism and Developmental Disorders. 2016 2016/10/01;46(10):3281-3294.

135. Khor AS, Melvin GA, Reid SC, et al. Coping, Daily Hassles and Behavior and Emotional Problems in Adolescents with High-Functioning Autism/Asperger's Disorder. Journal of autism and developmental disorders. 2013;44(3):593-608.

136. Bauminger N. The expression and understanding of jealousy in children with autism. Development and psychopathology. 2004;16(1):157-177.

137. Bury SM, Flower RL, Zulla R, et al. Workplace Social Challenges Experienced by Employees on the Autism Spectrum: An International Exploratory Study Examining Employee and Supervisor Perspectives. Journal of autism and developmental disorders. 2020.

138. Reijntjes A, Stegge H, Terwogt M. Children's coping with peer rejection: The role of depressive symptoms, social competence, and gender. Infant and Child Development. 2006 01/01;15:89-107.

139. Long-term effects of avoidant coping on adolescents' depressive symptoms [Internet]. Germany: Springer; 2000

140. Frydenberg E, Lewis R. Relations among well-being, avoidant coping, and active coping in a large sample of Australian adolescents. Psychological reports. 2009 07/01;104:74558.

141. Renty J, Roeyers H. Individual and Marital Adaptation in Men with Autism Spectrum Disorder and their Spouses: The Role of Social Support and Coping Strategies. Journal of Autism and Developmental Disorders. 2007;37(7):1247-1255.

142. Ahlers KP, Gabrielsen TP, Lewis D, et al. Supporting individuals with autism spectrum disorder in understanding and coping with complex social emotional issues. School psychology international. 2017;38(6):586-607.

143. Hull L, Petrides KV, Allison C, et al. "Putting on My Best Normal": Social Camouflaging in Adults with Autism Spectrum Conditions. Journal of autism and developmental disorders. 2017;47(8):2519-2534.

144. Groden J, Diller A, Bausman M, et al. The Development of a Stress Survey Schedule for Persons with Autism and Other Developmental Disabilities. Journal of autism and developmental disorders. 2001;31(2):207-217. 
145. Richards J. Examining the exclusion of employees with Asperger syndrome from the workplace. Personnel Review. 2012;41(5):630-646.

146. Courtenay K, Perera B. COVID-19 and People with Intellectual Disability: impacts of a pandemic. Irish journal of psychological medicine. 2020:1-6.

147. Pellicano E, Stears M. The hidden inequalities of COVID-19. Autism : the international journal of research and practice. 2020;24(6):1309-1310.

148. Bal VH, Wilkinson E, White LC, et al. Early Pandemic Experiences of Autistic Adults: Predictors of Psychological Distress. Autism research. 2021.

149. Cassidy SA, Nicolaidis C, Davies B, et al. An Expert Discussion on Autism in the COVID-19 Pandemic. Autism in Adulthood. 2020 2020/06/01;2(2):106-117.

150. Nicholls D. Qualitative research: Part one - Philosophies. International Journal of Therapy and Rehabilitation. 2009;16(10):526-533.

151. Smith JA, Osborn M. Interpretative phenomenological analysis. Qualitative psychology: A practical guide to research methods. Thousand Oaks, CA, US: Sage Publications, Inc; 2003. p. 51-80.

152. Weaver K, Olson JK. Understanding paradigms used for nursing research. Journal of advanced nursing. 2006;53(4):459-469.

153. Nicholls D. Qualitative research: Part one - Philosophies. International Journal of Therapy and Rehabilitation. 2009;16(10):526--533.

154. Illing J. Thinking About Research: Theoretical Perspectives, Ethics and Scholarship. 2013:329--347.

155. Bunniss S, Kelly DR. Research paradigms in medical education research. Medical education. 2010;44(4):358-366.

156. Willis J. A Recursive, Reflective Instructional Design Model Based on ConstructivistInterpretivist Theory. Educational Technology. 1995;35(6):5--23.

157. Dewe P. The Transactional Model of Stress: Some Implications for Stress Management Programs. Asia Pacific Journal of Human Resources. 1997;35(2):41--51.

158. Lyons A, Leon S, Phelps C, et al. The Impact of Child Symptom Severity on Stress Among Parents of Children with ASD: The Moderating Role of Coping Styles. Journal of Child and Family Studies. 2010 08/01;19:516-524.

159. Parsons D, Cordier R, Lee H, et al. Stress, Coping, and Quality of Life in Families with a Child with ASD Living Regionally. Journal of Child and Family Studies. 2020 2020/02/01;29(2):546-558.

160. McKenna HP. Theory and research: a linkage to benefit practice. (0020-7489 (Print)).

161. Smith J, Flowers P, Larkin M. Interpretative Phenomenological Analysis: Theory, Method and Research. Vol. 6. 2009.

162. Pringle J, Drummond J, McLafferty E, et al. Interpretative phenomenological analysis: a discussion and critique. Nurse researcher. 2011;18(3):20-24.

163. Smith JA, Shinebourne P. Interpretative phenomenological analysis. American Psychological Association; 2012.

164. Patton M. Qualitative Research And Evaluation Methods. http://lst-iiepiiepunescoorg/cgi-bin/wwwi32exe/[in=epidoclin]/?t2000=018602/(100). 2002;3.

165. Polit D, Beck C. Nursing research: generating and assessing evidence for nursing practice. Ninth Edition ed. Philadelphia: Wolters Kluwer Health/Lippincott Williams \& Wilkins; 2012.

166. Hennink MM, Kaiser BN, Marconi VC. Code Saturation Versus Meaning Saturation: How Many Interviews Are Enough? Qualitative Health Research. 2016 2017/03/01;27(4):591-608. 
167. Moon MD, Wolf LA, Baker K, et al. Evaluating Qualitative Research Studies for Use in the Clinical Setting. Journal of Emergency Nursing. 2013 2013/09/01/;39(5):508-510.

168. Roux AM, Rast JE, Shattuck PT. State-Level Variation in Vocational Rehabilitation Service Use and Related Outcomes Among Transition-Age Youth on the Autism Spectrum. Journal of autism and developmental disorders. 2018;50(7):2449-2461.

169. Wehman P, Lau S, Molinelli A, et al. Supported Employment for Young Adults With Autism Spectrum Disorder: Preliminary Data. Research and Practice for Persons with Severe Disabilities. 2012 11/01;37.

170. Wilkerson JM, Iantaffi A, Grey JA, et al. Recommendations for internet-based qualitative health research with hard-to-reach populations. Qualitative health research. 2014;24(4):561-574.

171. Ayling R, Mewse AJ. Evaluating internet interviews with gay men. (1049-7323 (Print)).

172. Mann C, Stewart F. Internet communication and qualitative research: A handbook for researching online. Sage; 2000.

173. Campbell MK, Meier A, Carr C, et al. Health Behavior Changes after Colon Cancer: A Comparison of Findings from Face-to-Face and On-Line Focus Groups. Family \& community health. 2001;24(3):88-103.

174. Archibald MM, Ambagtsheer RC, Casey MG, et al. Using Zoom Videoconferencing for Qualitative Data Collection: Perceptions and Experiences of Researchers and Participants. International journal of qualitative methods. 2019;18:160940691987459.

175. Lindsay S, Cagliostro E, Leck J, et al. Disability disclosure and workplace accommodations among youth with disabilities. 2019 (1464-5165 (Electronic)).

176. Hospital HBKRH. Youth Mentorship Program 2020 [cited 2020 November 20, 2020]. Available from: https://hollandbloorview.ca/services/programs-services/youthmentorship-program

177. Sibeoni J, Chambon L, Pommepuy N, et al. Psychiatric care of children with autism spectrum disorder - What do their siblings think about it? A qualitative study. Autism. 2019;23(2):326--337.

178. Mulhall A. In the field: notes on observation in qualitative research. Journal of Advanced Nursing. 2003;41(3):306-313.

179. Guillemin MaGL. Ethics, reflexivity, and Ethically important moments in research. Qualitative Inquiry. 2004;10(2):261--280.

180. Federal Policy for the Protection of Human Subjects ('Common Rule'). 2001.

181. Tracy SJ. Qualitative Quality: Eight Big-Tent Criteria for Excellent Qualitative Research. Qualitative Inquiry. 2010;16(10):837--851.

182. Pietkiewicz I, Smith J. A practical guide to using Interpretative Phenomenological Analysis in qualitative research psychology. CPPJ. 2014 01/01;20:7-14.

183. Fereday J, Muir-Cochrane E. Demonstrating rigor using thematic analysis: A hybrid approach of inductive and deductive coding and theme development. International journal of qualitative methods. 2006;5(1):80-92.

184. Swain J. A hybrid approach to thematic analysis in qualitative research: Using a practical example. SAGE Publications Ltd; 2018.

185. Yukhymenko MA, Brown SW, Lawless KA, et al. Thematic analysis of teacher instructional practices and student responses in middle school classrooms with problembased learning environment. Global Education Review. 2014;1(3):93-110.

186. $\mathrm{Xu} \mathrm{W}$, Zammit K. Applying thematic analysis to education: A hybrid approach to interpreting data in practitioner research. International Journal of Qualitative Methods. 2020;19:1609406920918810. 
187. Sydor A. An interpretative phenomenological analysis of young men's experiences of addressing their sexual health and the importance of researcher reflexivity. Journal of research in nursing. 2019;24(1-2):36-46.

188. Peat G, Rodriguez A, Smith J. Interpretive phenomenological analysis applied to healthcare research. Evidence-based nursing. 2019;22(1):7-9.

189. Krefting L. Rigor in Qualitative Research: The Assessment of Trustworthiness. The American journal of occupational therapy. 1991;45(3):214-222.

190. Korstjens I, Moser A. Series: Practical guidance to qualitative research. Part 4: Trustworthiness and publishing. The European journal of general practice. 2017;24(1):120-124.

191. Vincent J, Fabri M. The Ecosystem of Competitive Employment for University Graduates with Autism. International journal of disability, development, and education. 2020:1-17.

192. Johnson TD, Joshi A. Disclosure on the Spectrum: Understanding Disclosure Among Employees on the Autism Spectrum. Industrial and organizational psychology. 2014;7(2):278-281.

193. Nesbitt S. Why and why not? Factors Influencing Employment for Individuals with Asperger Syndrome. Autism : the international journal of research and practice. 2000;4(4):357-369.

194. Hurley-Hanson AE, Giannantonio CM, Griffiths AJ. Autism in the workplace : creating positive employment and career outcomes for Generation A. Cham, Switzerland: Palgrave Macmillan; 2020. (Palgrave explorations in workplace stigma).

195. Lindsay S, McDougall C, Sanford R. Disclosure, accommodations and self-care at work among adolescents with disabilities. Disability and rehabilitation. 2013;35(26):22272236.

196. Scholl L, Mooney M. Youth with Disabilities in Work-Based Learning Programs: Factors that Influence Success. Journal for Vocational Special Needs Education. 2004;26::4-16

197. Shaw KL, Hackett J, Southwood TR, et al. The Prevocational and Early Employment Needs of Adolescents with Juvenile Idiopathic Arthritis: the Adolescent Perspective. The British journal of occupational therapy. 2006;69(3):98-105.

198. Kim D, Son J, Vance ML. Preparing for the Future IT Era: Perceptions of Students with Disabilities About IT Training in South Korea. Journal of Postsecondary Education and Disability. 2012;25(4):297 - 308.

199. Kaushansky D, Cox J, Dodson C, et al. Living a secret: Disclosure among adolescents and young adults with chronic illnesses. Chronic illness. 2017;13(1):49-61.

200. Lindsay S, Cagliostro E, Carafa G. A systematic review of workplace disclosure and accommodation requests among youth and young adults with disabilities. Disability and Rehabilitation. 2018;40(25):2971-2986.

201. Browne DJ, Waghorn G. Employment services as an early intervention for young people with mental illness: Employment services as early intervention. Early intervention in psychiatry. 2010;4(4):327-335.

202. Hutchinson NL, Versnel J, Chin P, et al. Negotiating accommodations so that work-based education facilitates career development for youth with disabilities. Work (Reading, Mass). 2008;30(2):123-136.

203. Bal MI, Sattoe JN, van Schaardenburgh NR, et al. A vocational rehabilitation intervention for young adults with physical disabilities: participants' perception of beneficial attributes. Child : care, health \& development. 2017;43(1):114-125. 
204. Bengisu M, Balta S. Employment of the workforce with disabilities in the hospitality industry. Journal of sustainable tourism. 2011;19(1):35-57.

205. Carrero J, Krzeminska A, Härtel CEJ. The DXC technology work experience program: disability-inclusive recruitment and selection in action. Journal of management \& organization. 2019;25(4):535-542.

206. Meacham H, Cavanagh J, Shaw A, et al. HRM practices that support the employment and social inclusion of workers with an intellectual disability. Personnel review. 2017;46(8):1475-1492.

207. Ashley D, Graf NM. The Process and Experiences of Self-Employment Among People With Disabilities: A Qualitative Study. Rehabilitation counseling bulletin. 2018;61(2):90100.

208. Remington A, Pellicano E. 'Sometimes you just need someone to take a chance on you': An internship programme for autistic graduates at Deutsche Bank, UK. Journal of management \& organization. 2019;25(4):516-534.

209. Morris M, Begel A, Wiedermann B. Understanding the Challenges Faced by Neurodiverse Software Engineering Employees: Towards a More Inclusive and Productive Technical Workforce. 2015.

210. Charmaz K. Disclosing illness and disability in the workplace. Journal of international education in business. 2010;3(1/2):6.

211. Stanley N, Ridley J, Harris J, et al. Disclosing disability in the context of professional regulation: a qualitative UK study. Disability \& society. 2011;26(1):19-32.

212. Shuey KM, Jovic E. Disability Accommodation in Nonstandard and Precarious Employment Arrangements. Work and occupations. 2013;40(2):174-205.

213. Tomczak MT. Employees With Autism Spectrum Disorders in the Digitized Work Environment: Perspectives for the Future. Journal of Disability Policy Studies. 2020 2021/03/01;31(4):195-205.

214. Santuzzi AM, Waltz PR, Finkelstein LM, et al. Invisible Disabilities: Unique Challenges for Employees and Organizations. Industrial and organizational psychology. 2014;7(2):204-219.

215. Härtel C, O'Connor J. Disabled persons in the workplace. In: Syed J, Ozbilgin M, editors. Managing Diversity and Inclusion. Los Angeles, CA, USA: Sage; 2015. p. 215-243.

216. Harmuth E, Silletta E, Bailey A, et al. Barriers and Facilitators to Employment for Adults With Autism: A Scoping Review. Annals of International Occupational Therapy. 2018 2018/04/01;1(1):31-40.

217. Walsh L, Lydon S, Healy O. Employment and Vocational Skills Among Individuals with Autism Spectrum Disorder: Predictors, Impact, and Interventions. Review journal of autism and developmental disorders. 2014;1(4):266-275.

218. Waisman-Nitzan M, Gal E, Schreuer N. Employers' perspectives regarding reasonable accommodations for employees with autism spectrum disorder. Journal of management \& organization. 2019;25(4):481-498.

219. Davidson J. 'It cuts both ways': A relational approach to access and accommodation for autism. Social science \& medicine (1982). 2010;70(2):305-312.

220. Buckley E, Pellicano E, Remington A. "The Real Thing I Struggle with is Other People's Perceptions": The Experiences of Autistic Performing Arts Professionals and Attitudes of Performing Arts Employers in the UK. Journal of autism and developmental disorders. 2021;51(1):45-59.

221. Baron-Cohen S, Leslie AM, Frith U. Does the autistic child have a "theory of mind" ? Cognition. 1985;21(1):37-46. 
222. Brewer N, Young RL, Barnett E. Measuring Theory of Mind in Adults with Autism Spectrum Disorder. Journal of autism and developmental disorders. 2017;47(7):19271941.

223. Soeker MS. A descriptive, qualitative study of the challenges that individuals with Autism Spectrum Disorder experience when transitioning from skills training programs into the open labor market in Cape Town, South Africa. Work (Reading, Mass). 2020;65(4):733-747.

224. Raymaker DM, Teo AR, Steckler NA, et al. "Having All of Your Internal Resources Exhausted Beyond Measure and Being Left with No Clean-Up Crew": Defining Autistic Burnout. Autism in Adulthood. 2020.;2(2):132-143.

225. Baldwin S, Costley D. The experiences and needs of female adults with high-functioning autism spectrum disorder. Autism : the international journal of research and practice. 2016;20(4):483-495.

226. Kreiser NL, White SW. ASD in Females: Are We Overstating the Gender Difference in Diagnosis? Clinical child and family psychology review. 2014;17(1):67-84.

227. Crompton CJ, DeBrabander K, Heasman B, et al. Double Empathy: Why Autistic People Are Often Misunderstood. Frontiers for Young Minds. 2021;9.

228. Milton DEM. On the ontological status of autism: the 'double empathy problem'. Disability \& Society. 2012;27(6):883-887.

229. Nagib W, Wilton R. Gender matters in career exploration and job-seeking among adults with autism spectrum disorder: evidence from an online community. Disability and rehabilitation. 2019:1-12.

230. Cai RY, Richdale AL. Educational Experiences and Needs of Higher Education Students with Autism Spectrum Disorder. Journal of autism and developmental disorders. 2016;46(1):31-41.

231. Black MH, Mahdi S, Milbourn B, et al. Perspectives of key stakeholders on employment of autistic adults across the united states, australia, and sweden [https://doi.org/10.1002/aur.2167]. Autism Research. 2019 2019/11/01;12(11):16481662.

232. Ybema JF, Koopman A, Peeters M. Working in Sheltered Employment: A Weekly Diary Study. International journal of stress management. 2020;27(2):160-171.

233. Flores N, Moret-Tatay C, Gutiérrez-Bermejo B, et al. Assessment of Occupational Health and Job Satisfaction in Workers with Intellectual Disability: A Job Demands-Resources Perspective. International journal of environmental research and public health. 2021;18(4):2072.

234. Diener ML, Wright C, A., Taylor C, et al. Dual perspectives in autism spectrum disorders and employment: Toward a better fit in the workplace. Work (Reading, Mass). 2020;67(1):223-237.

235. Parr AD, Hunter ST. Enhancing work outcomes of employees with autism spectrum disorder through leadership: Leadership for employees with autism spectrum disorder. Autism : the international journal of research and practice. 2014;18(5):545-554.

236. Anda RF, Felitti VJ, Bremner JD, et al. The enduring effects of abuse and related adverse experiences in childhood. A convergence of evidence from neurobiology and epidemiology. European archives of psychiatry and clinical neuroscience. 2006;256(3):174-186.

237. Edwards VJ, Holden Gw Fau - Felitti VJ, Felitti Vj Fau - Anda RF, et al. Relationship between multiple forms of childhood maltreatment and adult mental health in community respondents: results from the adverse childhood experiences study. (0002-953X (Print)). 
238. Schilling EA, Aseltine RH, Jr., Gore S. Adverse childhood experiences and mental health in young adults: a longitudinal survey. BMC public health. 2007;7:30-30.

239. Taylor JL, Corbett BA. A review of rhythm and responsiveness of cortisol in individuals with autism spectrum disorders. Psychoneuroendocrinology. 2014 2014/11/01/;49:207228.

240. Mehtar M, Mukaddes NM. Posttraumatic Stress Disorder in individuals with diagnosis of Autistic Spectrum Disorders. Research in Autism Spectrum Disorders. 2011 2011/01/01/;5(1):539-546.

241. Southall K, Gagné J-P, Jennings MB. Stigma: A negative and a positive influence on help-seeking for adults with acquired hearing loss. International Journal of Audiology. 2010 2010/11/01;49(11):804-814.

242. Ellison ML, Russinova Z, MacDonald-Wilson KL, et al. Patterns and correlates of workplace disclosure among professionals and managers with psychiatric conditions. Journal of vocational rehabilitation. 2003;18:3-13.

243. Restubog SLD, Ocampo ACG, Wang L. Taking control amidst the chaos: Emotion regulation during the COVID-19 pandemic. Journal of vocational behavior. 2020;119:103440-103440.

244. Kniffin KM, Narayanan J, Anseel F, et al. COVID-19 and the workplace: Implications, issues, and insights for future research and action. American Psychologist. 2021;76(1):63.

245. Solomon S. Home is where the job is: How COVID-19 is changing the workplace. The Times of Israel. 2020. Available from: https://www.timesofisrael.com/home-is-wherethe-job-is-how-covid-19-is-changing-the-workplace/

246. Goldfarb Y, Gal E, Golan O. Implications of Employment Changes Caused by COVID19 on Mental Health and Work-Related Psychological Need Satisfaction of Autistic Employees: A Mixed-Methods Longitudinal Study. 2021 (1573-3432 (Electronic)).

247. Anderson AH, Stephenson J, Carter M. Perspectives of Former Students with ASD from Australia and New Zealand on Their University Experience. Journal of autism and developmental disorders. 2020;50(8):2886-2901.

248. Beck KB, Conner CM, White SW, et al. Mindfulness "Here and Now": Strategies for Helping Adolescents With Autism. Journal of the American Academy of Child and Adolescent Psychiatry. 2020;59(10):1125-1127.

249. Dipeolu AO, Storlie C, Johnson C. College Students With High $\square$ Functioning Autism Spectrum Disorder: Best Practices for Successful Transition to the World of Work. Journal of college counseling. 2015;18(2):175-190.

250. Mantler J, Matejicek A Fau - Matheson K, Matheson K Fau - Anisman H, et al. Coping with employment uncertainty: a comparison of employed and unemployed workers. (1076-8998 (Print)).

251. Holton MK, Barry AE, Chaney JD. Employee stress management: An examination of adaptive and maladaptive coping strategies on employee health. Work (Reading, Mass). 2016;53(2):299-305.

252. Graham J, Albery IP, Ramirez AJ, et al. How hospital consultants cope with stress at work: implications for their mental health [https://doi.org/10.1002/smi.884]. Stress and Health. 2001 2001/03/01;17(2):85-89.

253. Lim J, Bogossian F Fau - Ahern K, Ahern K. Stress and coping in Australian nurses: a systematic review. (1466-7657 (Electronic)).

254. Perreault M, Touré EH, Perreault N, et al. Employment Status and Mental Health: Mediating Roles of Social Support and Coping Strategies. Psychiatric Quarterly. 2017 2017/09/01;88(3):501-514. 
255. Assessment in sports: psychological and neuropsychological approaches. 2019.

256. Althaus VaKJ-LaGV. Critical review of theoretical models linking work environment, stress and health: Towards a meta-model. Travail Humain. 2013;76(2):81--103.

257. Mark GaSA. Stress models: A review and suggested new direction. Occupational health psychology: European perspectives on research, education, and practice. 2008;3:11--144.

258. Liew SM, Thevaraja N, Hong RY, et al. The Relationship Between Autistic Traits and Social Anxiety, Worry, Obsessive-Compulsive, and Depressive Symptoms: Specific and Non-specific Mediators in a Student Sample. Journal of autism and developmental disorders. 2015;45(3):858-872.

259. Yang T, Liu J, Zhang Y, et al. Coping style predicts sense of security and mediates the relationship between autistic traits and social anxiety: Moderation by a polymorphism of the FKBP5 gene. Behavioural brain research. 2021;404:113142-113142.

260. Campbell-Sills L, Barlow DH, Brown TA, et al. Acceptability and Suppression of Negative Emotion in Anxiety and Mood Disorders. Emotion (Washington, DC). 2006;6(4):587-595.

261. Cai RY, Richdale AL, Dissanayake C, et al. How Does Emotion Regulation Strategy Use and Psychological Wellbeing Predict Mood in Adults With and Without Autism Spectrum Disorder? A Naturalistic Assessment. Journal of autism and developmental disorders. 2020;50(5):1786-1799.

262. Austin RD, Sonne T. The dandelion principle: redesigning work for the innovation economy. MIT Sloan management review. 2014;55(4):67.

263. Aspinall D. Op-ed: Two-thirds of our team is autistic. That's helping us survive the coronavirus CNBC2020. Available from: https:/www.cnbc.com/2020/04/16/our-it-teamis-autistic-thats-helping-us-survive-the-coronavirus.html

264. Kerns CM, Newschaffer CJ, Berkowitz SJ. Traumatic Childhood Events and Autism Spectrum Disorder. Journal of Autism and Developmental Disorders. 2015 2015/11/01;45(11):3475-3486.

265. Chen JL, Sung C, Pi S. Vocational Rehabilitation Service Patterns and Outcomes for Individuals with Autism of Different Ages. Journal of autism and developmental disorders. 2015;45(9):3015-3029.

266. Collins CS, Stockton CM. The Central Role of Theory in Qualitative Research. International Journal of Qualitative Methods. 2018 2018/12/01;17(1):1609406918797475.

267. Osgood DW, Ruth G, Eccles JS, et al. Six Paths to Adulthood: Fast Starters, Parents without Careers, Educated Partners, Educated Singles, Working Singles, and Slow Starters. Chicago: University of Chicago Press; 2019. p. 320-355. 


\section{Appendices}

\section{Appendix A:}

\section{Appendix A: Information Consent Form}

\section{(Link back to section 3.5.2)}

\section{Blcorview}

RESEARCH INSTITUTE

March 26, 2020

\section{Partners for enhancing healthy and productive work for young men and women with disabilities}

My name is Abby Vijayakumar. I part of a research team at Bloorview Research Institute and Holland Bloorview Kids Rehabilitation Hospital. We want to understand what it is like for young people with disabilities who are employed or are looking for work. This study is funded by the Canadian Institutes of Health Research and the Social Sciences and Humanities Research Council. To help you decide if you would like to participate, the sections below answer questions you may have.

\section{What is this study about?}

This study will explore the experience of youth with disabilities as they apply for work or their current work experiences. It also explores the experiences of employers and other knowledge users. We are currently inviting 25 young people with autism spectrum disorder to take part and share their experiences, as part of a broader study on youth with disabilities. You may also choose to participate in one of our 5 webinars or 2 workshops (approx. 50 participants each) on disability disclosure and accommodations. The research interview will allow you to tell us more about your experiences. We will be also interviewing 30 employers about their experiences in hiring people with disabilities.

We want to learn about your experience in disability disclosure and asking for accommodations in the workplace. We want to understand the process involved and what recommendations you may have. This information will help us make sure that young people have the support that they need.

\section{How will I be involved in this study?}

You can be involved in this research study if you are a:

- 15-30 years old, and have autism spectrum disorder

- able to speak English

- employed or have work experience 
If you meet these criteria, and would like to share more about your transition experiences, you are invited to participate in an interview. If you are interested in participating, we will arrange for a researcher to follow-up with you. Due to the recent outbreak of COVID-19, interviews will be conducted remotely (e.g., over the phone or through Zoom) until further notice. The interview will last about 30-60 minutes and will be audio recorded.

The interview questions include:

- What is your experience of applying for employment, disclosing your disability and asking for accommodations?

- Which services, people, or practices helped you to prepare for employment?

- What were the key successes or challenges in this process?

- What advice do you have for other youth with disabilities who are looking for work?

We will ask these and other questions to help us understand your transition experiences. At the end of the interview we will summarize the key points of what you said and ask you if it is accurate.

\section{What else should I know?}

If you participate in the interview, we will give you a $\$ 10$ gift card as thanks for participating. In addition, we can also offer volunteer hours for participating. We will also cover your travel costs (day parking pass at Holland Bloorview or TTC tokens) if you are travelling to meet with us.

\section{Who will see the information that is collected?}

For your information, your interview transcript will be identified by a number, not your name. Only the members of the research team will have access to the file linking your information to your transcript. Paper copies of the transcript will be kept in a locked cabinet in my office at Holland Bloorview. All the information about you will be kept in a locked cabinet. This information can only be seen by the Research Team who are not involved in your clinical care.

We are audio recording the interview to accurately capture your experiences. You will need to agree to be audio recorded before you can participate in the interview. We will take several steps to protect your privacy and confidentiality. These include encrypting the audio files and password protecting them. We will also de-identify the transcripts. We will delete the audio recordings after they have been verified.

No information that could reveal who you are will be shared with anyone without your permission unless required by law. Your name will not be included in the transcripts we type up from the interviews. We will securely destroy all the information collected about you. We will delete any information that could identify you at the end of the study. We will keep de-identified transcripts for seven years after the study closure.

\section{Do I have to participate in this study?}

Participation in this study is voluntary. You don't have to take part in this study. You also have the right to change your mind and stop participating at any time. You can choose not to answer a question without providing a reason. You can tell the researcher conducting the interview or contact one of the team members listed below. For the interview, you may withdraw your 
consent during or after the interview and it will be deleted and not included in the study. This will no longer be possible once the results have been analyzed and submitted for publication. Participating or not participating will not affect the services you receive from Holland Bloorview.

\section{What are the risks and benefits?}

Some youth may enjoy the opportunity to give feedback about their experience of looking for work or being employed. Others may not. Some may think the study is taking too much of their time. We think this project will help us learn how to support young people with their transition to employment.

By consenting to take part you have not waived any rights to legal recourse in the event of research-related harm. Some participants may become upset when thinking about their transition experiences. If this happens a member of our team will refer you to someone that you can speak to about this further.

\section{Sharing?}

The results of this study may be published and presented. Quotes may be used from your interview to help explain what we learned from the study but no real names will be used. We will ensure that we do not reveal the identity of any of the participants. Results may be used to help inform Phase 2 of the project and policies around workplace disclosure and accommodations. We will send you a summary letter at the end of the study. If interested, you may request a copy of the final research report.

\section{What if I have questions?}

Please ask me to explain anything you don't understand before signing the consent form. My phone is 416-425-6220 x 3654. You can also contact the Research Ethics Board at Holland Bloorview (416-4256220 x3507; email: reb@hollandbloorview.ca) to discuss any concerns about your rights as a research participant. Thank you for thinking about helping us with this project.

Yours truly,

On behalf of the Research Team

Sally Lindsay, PhD, Senior Scientist

Bloorview Research Institute, Holland Bloorview Kids Rehabilitation Hospital \& University of Toronto

(416) 425-6220 x3654 slindsay@,hollandbloorview.ca

Jennifer Stinson, PhD, Associate Professor, Faculty of Nursing, Sick Kids \& University of Toronto

Mary Stergiou-Kita, PhD, Assistant Professor, Dept. Occupational Science \& Occupational Therapy, University of Toronto

Joanne Leck, PhD, Professor, Telfer School of Management, University of Ottawa 
Winny Shen, PhD, Assistant Professor, Industrial/Organizational Psychology, University of Waterloo 


\section{Blcorview}

\section{RESEARCH INSTITUTE}

\section{PARTICIPANT CONSENT FORM: INTERVIEW [youth]}

\section{Partners for enhancing healthy and productive work for young men and women with disabilities}

Please complete this form and return it to the Researcher.

I have read the information letter dated March 26, 2020. I understand what this study is about.

I have been informed that the information collected through this interview will be used to understand the disability disclosure and accommodation processes. I have been informed that this information may help support employers and youth with disabilities.

I agree to participate in this study. I understand that I may drop out of the study at any time. I agree to be audio-taped during the interview.

Participant's Signature

Researcher's signature

Date

Participant's Name (Please Print) 


\title{
Appendix B:
}

\section{Appendix B: Interview Guide}

\section{(Link back to section 3.6.2)}

\author{
Interview guide (youth)
}

\section{Demographics}

1. I would first like to ask you some demographic questions

What is your gender?

What type of disability do you have?

2. What is your employment status?

- (employed (part / full time) / volunteering (if yes, probe for the following):

- Could you tell me a bit about where you work?

- name of employer (type and size of company and location)

- How long have you been working for XX company?

- How did you find the job?

- Please tell me about any accommodations that you asked for (e.g., flexible time, accessible workspace etc.)?

- How did you ask for them and when

- Can you describe what you do on a typical day?

○ What do you like most about working / volunteering here? (probe for inclusion)

- Do you have any opportunities for professional development or advancement? (probe for type of opportunities; or why lack of opportunities)

○ Do you feel included, respected, and valued by your employer and coworkers? (if no, probe why not).

- Tell me about what it is you do here?

- Can you describe what you do on a typical day?

- What do you like most about working here?

- So, do you work by yourself or in a team?

- What are some things that you do not enjoy?

- E.g. noise, lighting, loud chatter, too many people

3. Have you experienced any challenges in finding employment? (probe for accessibility, accommodations, discrimination, disability disclosure)

Probing questions

- What are the challenges you face when you need to complete work tasks in a timely manner? 
- Are there any tasks that require accommodations from your supervisor?

- If there are, can you give me an example?

- If they mentioned accommodations

- What are/were the challenges you faced when asking for accommodations?

- If they mention challenges( accommodations, work-related challenges, etc.):

- How do/did these challenges make you feel?

- Do these challenges lead to you feeling stressed or anxious?

- How do you cope with meeting work demands?

- Do you feel like these challenges affect your employment (e.g. your productivity)

○ How so?

- What do you do to overcome these challenges?

- What are some ways you manage/cope with this stress?

- Probe for problem-based coping or emotion-based coping

- Could you give me an example of what you did?

- If they mention one coping strategy:

- What are some other ways you cope?

- If they mention multiple strategies:

- How would you utilize your stress management strategies?

- When would you utilize these stress management strategies (e.g. type of situations)

- How do you decide on which strategy to utilize?

4. did you disclose your disability to your employer / potential employers?

a. If not, ask why and what are the potential challenges when disclosing a disability?

b. (if so, how and when did you do this?)

c. why did you decide to disclose?

d. How did you feel when you disclosed your disability?

e. What accommodations did you ask for

f. Prompting questions

How would you describe your relationship with your co-workers and supervisors?

- How do you communicate with your peers?

- What are the challenges you face when you communicate with your co-workers?

- Do you feel included in your work?

- e.g. your greetings are answered at work, others have conversations with you in a group setting, others invite you to lunch (WOS Scale) 
- If there are no peer supports, how do you cope with stressful circumstances?

Do you feel like you can bring up your concerns and challenges to your supervisor?

5. do you feel that being a man / woman influences your employment experiences (and/or ability to find work / ask for accommodations)?

- Prompting questions

- Tell me about a time where you felt like you got treated differently because of your gender?

- How does your gender influence the way you get treated at work?

- What are some ways you overcome these challenges?

- Prompt for coping mechanisms

6. What advice do you have for others who have a disability who may be having a difficult time finding employment?

7. Is there anything else that you would like to mention that we did not get a chance to talk about? 


\section{Appendix C:}

\section{Appendix C: Journal Template}

(Link back to section 3.6.2)

Journal Template:

Demographic Info

Societal Pressures:

Emotions/feelings due to challenges:

$\underline{\text { Notes/reflection/observations }}$ 


\section{Appendix D:}

\section{Appendix D: Ethics Approval}

(Link back to section 3.7)

\section{Holland Blcorview}

\author{
Kids Rehabilitation Hospital
}

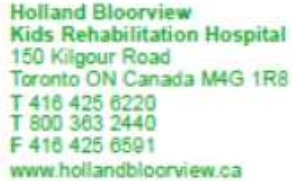

Holland Bloorview Kids Rehabilitation Hospital 150 Kilgour Road Toronto ON Canada MAG 1R8 T 4164256220 T 8003032440 www hollandbloorview ca

A teaching hospital fully affliliated with the University of Toronto

\section{Holland Bloorview Research Ethics Board Renewal Approval Notification}

Hol and Bloorview Research Ethics Board operates in compliance with, and is constituted in accordance with. the requirements of the Tri-Council Policy Statement: Ethical Conduct for Research Involving Humans (TCPS 2), the International Conference on Hamonisation Good Clinica Practice Consolidated Guideline (ICH GCP). Part C. Division 5 of the Food and Drug Regulations; Part 4 of the Natural Health Products Regulations; Part 3 of the Medical Devices Regulations and the provisions of the Ontario Personal Health information Protection Act (PH.PA 2004) and its applicable regulations. Holand Bloorvew Research Ethics Board is qualfied through the CTO REB Qualification Program and is registered with the US. Department of Heath and Human Services (DHHS) OFfice for Human Research Protection (OHRP).

Study Title: Partners for enhancing healthy and productive work for young men and women with

disabilities

File number: $16-639$

Principal Investigator: Sally Lindsay

Co-Investigators: Jennifer Stinson, Joanne Leck, Winny Shen, Mary Stergiou-Kita

Original Approval Date: July 22, 2016

New Expiry Date: June 30, 2021

Review Type: Delegated

Dear Dr. Lindsay,

The Holland Bloorview Research Ethics Board (REB) has extended the ethics approval of the above named study for an additional year ending June 30, 2021.

This study must continue to be conducted in accordance with the description in the application and any supplementary documents for which ethics approval has been granted. The REB needs to be notified of any unanticipated or unintentional divergence or departures from the protocol through a "Protocol Deviation Form". Any intentional changes to the protocol need be submitted through an "Amendment Form" to the REB for approval before the changes are implemented, except where necessary to eliminate immediate hazards to the participants.

Any unanticipated problems that occur as a result of your study must be reported to the REB by submitting an "Adverse Event/Unanticipated Problem Form".

If the study is expected to continue beyond the new expiry date, you must request another renewal, at least thirty days prior to the expiry date, by submitting an "Annual Renewal Form". When the study is completed or terminated, you need to submit a "Study Closure Form" to the REB.

Sincerely,

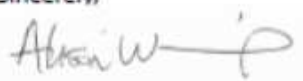

Alison Williams, PhD

Chair, Research Ethics Board

Holland Bloorview Kids Rehabilitation Hospital

P: $4164256220 \times 3248$

awilliams@hollandbloorview.ca 


\section{Appendix E:}

\section{Appendix E: Sample Theme Table}

\section{(Link back to section 3.8.1)}

\begin{tabular}{|c|c|}
\hline Themes in the interviews & Interview \# \\
\hline $\begin{array}{l}\text { 1. Stressors (stress inducing situations) } \\
\text { a. Impact of stress } \\
\text { b. Feelings of unfamiliarity } \\
\text { 2. Salient Workplace Factors } \\
\text { a. Bringing up challenges } \\
\text { b. Bringing up concerns, } \\
\text { awkward } \\
\text { c. Risks to disability disclosure } \\
\text { 3. Salient Workplace Factors } \\
\text { a. Freedom to ask questions } \\
\text { b. Flexibility } \\
\text { c. Special interests/goals } \\
\text { d. Accommodations } \\
\text { e. Management Styles/ employer } \\
\text { f. Professional development } \\
\text { g. Positive workplace } \\
\text { h. Environment } \\
\text { Empowerment through } \\
\text { Coping strengths focus } \\
\text { a. Emotion Coping } \\
\text { b. Coping w/communication } \\
\text { c. Coping on own }\end{array}$ & Interview 1 \\
\hline $\begin{array}{l}\text { 1. Stressors (stress inducing situations) } \\
\text { a. Pandemic } \\
\text { b. Social interactions } \\
\text { c. Work tasks } \\
\text { 2. Salient Workplace Factors } \\
\text { a. Accommodations } \\
\text { b. Challenges finding } \\
\text { employment } \\
\text { c. Disability disclosure } \\
\text { 3. Salient Workplace Factors } \\
\text { a. Special interests } \\
\text { b. Accommodations } \\
\text { c. Disability disclosure } \\
\text { d. Inclusion } \\
\text { e. Respected and valued } \\
\text { f. Preventative stress measures }\end{array}$ & Interview 2 \\
\hline
\end{tabular}




\begin{tabular}{|c|c|}
\hline $\begin{array}{l}\text { g. Workplace } \\
\text { environment/employer } \\
\text { relations/employer attitudes } \\
\text { 4. Coping } \\
\text { a. Types of coping } \\
\text { b. Importance of coping }\end{array}$ & \\
\hline $\begin{array}{l}\text { 1. Stressors (stress inducing situations) } \\
\text { a. Impact of stress } \\
\text { 2. Salient Workplace Factors } \\
\text { a. Challenges finding } \\
\text { employment } \\
\text { b. Taking longer to complete } \\
\text { tasks } \\
\text { c. Accessing devices } \\
\text { 3. Salient Workplace Factors } \\
\text { a. Accommodations } \\
\text { b. Freedom/flexibility } \\
\text { c. Included and valued } \\
\text { d. Special interests/making a } \\
\text { difference } \\
\text { e. Stress preventative measures } \\
\text { f. Disability disclosure (no } \\
\text { discrimination, hiring } \\
\text { inclusively) (+tive) } \\
\text { g. Work environment } \\
\text { Coping } \\
\text { a. Flexibility to cope } \\
\text { b. Emotion cope } \\
\text { c. Impact of coping }\end{array}$ & Interview 3 \\
\hline
\end{tabular}




\section{Appendix F}

\section{Appendix F: Sample Coding}

(Link back to section 4.1)

\section{Sample Coding for Subtheme- Risks to Disability Disclosure from NVivo:}

$<$ Files \\Terview $\backslash \backslash Y 1>-\S 2$ references coded [1.05\% Coverage]

Reference 1 - 0.59\% Coverage

The inability to control how the other person responds. Umm... what's the question? [AV repeats] Uhhh ya I guess brave enough and being self-assured self-confident enough to actively disclose it, then be prepared to admit that...the struggle.

Reference 2 - $0.46 \%$ Coverage

can cause some type of stress and anxiety or overwhelming feeling for someone for some individuals?

Y1: For sure yes, definitely. It's definitely a source of... self-conscious insecurity

$<$ Files $\backslash \backslash$ Terview $\backslash \backslash Y 10>-\S 2$ references coded [0.74\% Coverage]

Reference $1-0.41 \%$ Coverage

Y10: like at that point, he knew how I was like in general, but in general if you're [inaudible] at your job interview, then obviously they'll have misconceptions and biased against you

\section{Reference $2-0.32 \%$ Coverage}

I wouldn't bother like...like what if I just had bad luck and that one person doing the interview has a bad impression about autism like why risk it...

\section{$<$ Files \\Terview $\backslash \backslash Y 11>-\S 3$ references coded [1.50\% Coverage]}

Reference $1-0.50 \%$ Coverage

but what are the potential challenges when disclosing a disability?

Y11: they would probably see me differently 
Reference $2-0.44 \%$ Coverage

Y11: [inaudible] about my disability, they would treat me differently, whether that's good or bad....

Reference 3 - $0.56 \%$ Coverage

AV: are there any other potential challenges that you can think of when disclosing a disability?

Y11: Mainly trust I guess

$<$ Files $\backslash$ Terview $\backslash \backslash Y 12>-\S 2$ references coded [0.93\% Coverage]

Reference 1 - $0.75 \%$ Coverage

Y12: umm once I actually get a job, if I them, by the way I'm on the autism spectrum, they can't do anything about it...they can't fire me over it or anything... but if I tell someone during a job interview... and they're like oh this person may not be a very good fit, they can just not hire me...you know like...there's nothing that I can do about that because there's no way to prove it...so you know

Reference 2 - $0.18 \%$ Coverage

AV: are you referring to potential...maybe discrimination without you even knowing? [Y12 says ya]

$<$ Files \\Terview $\backslash \backslash Y 2>-\S 1$ reference coded [0.84\% Coverage]

Reference 1 - 0.84\% Coverage

I think the best ways is ...just to have that feeling that...you can bring something up to your boss without fear of retribution like say hey...we should...you should be able to solve this in a different way or you should talk to this coworker about such and such you know or whatever you know without fear of being fired or punished for saying...for speaking out.

$<$ Files\\Terview\\Y6> - § 7 references coded [2.53\% Coverage]

Reference $1-0.23 \%$ Coverage 
Reference 2 - 0.36\% Coverage

I don't disclose it a lot because people are ablest as hell and every response I get is basically like "no you're not, you're nothing like my sister's friend's 4 year old child" and I'm like "is that because they are 4 ? Is it because they are 4 and I'm 30 , is that why I'm nothing like

\section{Reference 3 - 0.28\% Coverage}

more hesitant about that...because she is a psychology professor umm... and oh boy there is a lot of problematic shit that's going around in psychology departments about autism and I'm not sure that I want to have that conversation

\section{Reference $4-0.16 \%$ Coverage}

Umm anyway, uhh ya umm...so that's a little bit more of a hurdle but she's really nice and one day I'm sure I'll get over that hurdle

\section{Reference $5-0.53 \%$ Coverage}

people's view of disabilities in particular developmental, or intellectual or communication da da da... is basically like, [voice changes to mocking] if you can disclose that you have a disability, you're too smart to have a disability. Umm which is very frustrating, there are so many risks to disclosure because... disclosure being safe and being beneficial is dependent on your boss not being abelist and in an ableist society,

\section{Reference $6-0.51 \%$ Coverage}

. So even if your boss is a really nice person, its still a huge fucking risk because they can be a nice person who also tantalizes autistic people in their head say you might be passed over for promotion because your boss who is well-meaning, but ultimately absorbed ableist messages might be like "well they're autistic, you know what if they can't handle it" umm or starts talking down to you like a child...

\section{Reference $7-0.46 \%$ Coverage}

Whereas growing up autistic in order to keep ourselves safe, we often have to hide these parts of ourselves and hide our reasons for doing things because people will not regard our reasons for 
how we feel or how we act as being valid reasons. Now I can actually say the actual reason why I need to opt out of something instead of making up neurotypically friendly lie

$<$ Files $\backslash$ Terview $\backslash Y$ Y7> - § 2 references coded [2.06\% Coverage]

Reference $1-0.83 \%$ Coverage

Y7: honestly I do that with a few companies, I've been doing that less as I think they might not be hiring... a lot of places may not be hiring based on that...but you know... who understands autism better than an autism advocacy group right? So it doesn't make sense not to...and that way, I can formally ask for accommodations if it came to that

Reference 2 - $1.22 \%$ Coverage

Y7: the thing is...I'm not sure because...I don't know if they're discriminating me based on that or its "I wrote down that I am bad at my job or something" [AV says okay] like its...its...intentionally obtuse... but umm...I guess I have tried not talking about my autism...I try talking about my autism but I also tried working on my resume... working on my interview ability...and... frankly I'm not even sure there was a job for me to have by the time I got to the interview, so it might even be that...but I don't know...

\section{$<$ Files \\erview $\backslash \backslash Y 8>-\S 1$ reference coded [0.78\% Coverage]}

\section{Reference $1-0.78 \%$ Coverage}

Like for everybody else, I actually told them at my own time because when it comes to me at interviews, I don't tell them right off the bat [AV says ya]. I'm one of those people that's like during interviews, I want to be treated like a normal person and not have that disclosed until I've been there for a while and I open up and then I'm able to tell them. That's just how I ...that's just how it works with me just because I'm not comfortable disclosing it to everybody and I don't think I ever will be just because I'm still learning it myself

\section{$<$ Files \\Terview $\backslash \backslash Y 9>-\S 3$ references coded [1.53\% Coverage]}

\section{Reference $1-0.23 \%$ Coverage}

but my original plan had been to not let it come out because I thought it would hurt me professionally and it has... it definitely hurt me professionally like she didn't keep me at that point 
Reference 2 - 0.65\% Coverage

Y9: I did...I contacted my union representative [AV says ya] and said it isn't okay but basically the advice I was given was I can report her... and I can probably sue the school board if I wanted to due to discrimination. The problem is if I do that, I will never be hired as a teacher again because I would be seen as a troublemaker, so I should weigh my choices very carefully, do I want to be a teacher or do I want to get justice for this particular situation and I decided that I didn't want to commit career suicide so...

Reference 3 - 0.65\% Coverage

, I'll have to see what they do because this is the first time I told them before there was an issue because before I thought it was none of their business they're going to discriminate me against it so I shouldn't tell them but then they always end up coming out because there's always some sort of issue because other people are sneaky and they have all these weird rules that they don't tell anybody, but they get upset when you don't follow them. How are you supposed to know what the rules were if they don't tell you... 